$$
\begin{gathered}
\text { NGT } 80001 \\
\text { IN-19-CR } \\
109723 \\
98 P
\end{gathered}
$$

\title{
APOLLO LIGHTCRAFT PROJECT
}

\section{PREPARED FOR:}

The NASA/USRA Advanced Design Program

3rd Annual Summer Conference

Washington, D. C.

June $17 \cdot 19,1987$

\section{INSTRUCTOR:}

Professor Leik N. Myrabo

Dept. of Mechanical Engrg., Aeronautical Engrg. and Mechanics

Rensselaer Polytechnic Institute, 4004 JEC

Troy, N. Y. 12180-3590; Phone (518) 276-6545

\section{STUDENT DESIGN GROUP:}

John S. Blandino, Chris A. Borkowski, David P. Cross, Scott R. Frazier, Stephen C. Hill, Todd J. Mitty, Jeffrey P. Moder, Ciro Morales, Gregory A. Nyberg, Michael V. Powers, Jacques C. Richard, Wayne L. Smith, T. Darton Strayer and Cliff L. Zaretzky.

\section{COVER PHOTO CREDIT:}

"Apollo Lightcraft accelerating in the rocket mode", by Randy Bradley of the RPI Center for Interactive Computer Graphics (CICG). The image was interactively modeled on an Evans and Sutherland PS300, then rendered on a DG-MV10000 using the Clockworks (CICG) software, and finally imaged on the Raster-Tech One/380 and recorded with a Dunn 635 camera. 


\section{TABLE OF CONTENTS}

LIST OF FIGURES $\ldots \ldots \ldots \ldots \ldots \ldots \ldots \ldots$ iii

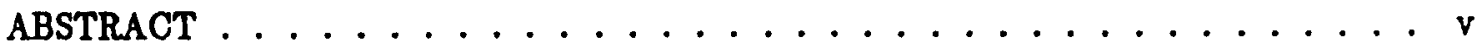

I. INTRODUCTION $\ldots \ldots \ldots \ldots \ldots \ldots \ldots \ldots \ldots \ldots$

A. Vehicle/Propulsion System Description . . . . . . . . . . . 1

B. Design Study and Final Report Organization $\ldots \ldots \ldots \ldots$

II. ERH THRUSTER MODE $\ldots \ldots \ldots \ldots \ldots \ldots \ldots \ldots$

A. Basic Operating Principles .............. 7

B. LSD Blast Wave Analysis . . . . . . . . . . . 8

C. Static Thrust Model . . . . . . . . . . . . . 12

D. Dynamic Thruster Model . . . . . . . . . . . . 14

E. Results . . . . . . . . . . . . . . . . 16

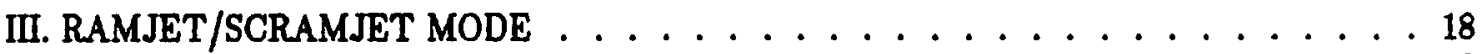

A. Inlet Spike . . . . . . . . . . . . . . . 18

B. Internal Diffuser $\ldots \ldots \ldots \ldots \ldots$

C. Combustion Chamber . . . . . . . . . . . . . . . . 19

D. Plug Nozzle . . . . . . . . . . . . . . . 19

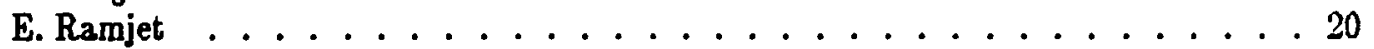

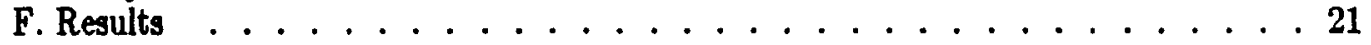

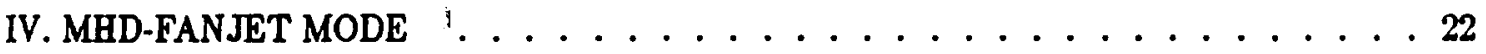

A. Laser Absorption Chamber $\ldots \ldots \ldots \ldots \ldots \ldots 2$

B. MHD Generator . . . . . . . . . . . . . . . 23

C. MHD Accelerator $\ldots \ldots \ldots \ldots \ldots$

D. MHD-Fanjet Performance . . . . . . . . . . . . . 33

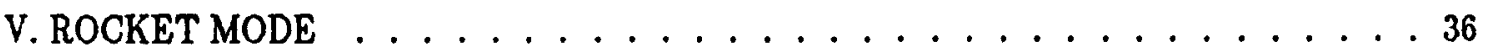

A. Rocket Performance $\ldots \ldots \ldots \ldots \ldots \ldots$

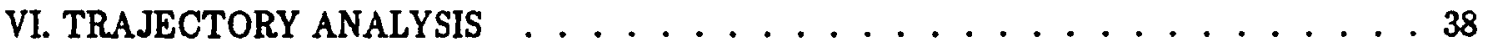

A. Vehicle and Environment Models . . . . . . . . . . . 38

B. Trajectory Simulation . . . . . . . . . . . . 40

C. Results . . . . . . . . . . . . . . . . 42

D. Summary . . . . . . . . . . . . . . . 45

VII. SUMMARY AND CONCLUSIONS $\ldots \ldots \ldots \ldots \ldots \ldots \ldots$

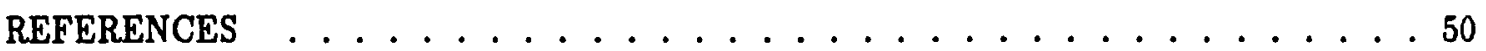

APPENDIX : THREE-DIMENSIONAL MODELING OF APOLLO LIGHTCRAFT LOFT LINES 


\section{LIST OF FIGURES}

Figure 1. The Apollo Lightcraft configuration (10-GW machine). . . . . . . . . . . . la Figure 2. Interior of the Apollo Command Module . . . . . . . . . . . . . . . la Figure 3. Dimensions for the Apollo Lightcraft. . . . . . . . . . . . . . . . 1b

Figure 4. Reentry configuration for the Apollo Lightcraft. . . . . . . . . . . . . . Ib

Figure 5. $10 \mathrm{GW}$ Satellite Solar Power Station . . . . . . . . . . . . . . . . $2 \mathrm{a}$

Figure 6. Apollo Lightcraft in ERH thruster mode. . . . . . . . . . . . . . . . . . . . $2 a$

Figure 7. Repetitively-pulsed planar heater for ramjet and scramjet modes. . . . . . . . . . 3a

Figure 8. Bow shock angle vs. shroud translation. . . . . . . . . . . . . . 3a

Figure 9. Diagram of MHD-fanjet cycle (an electric air-turborocket) . . . . . . . . . . . 3b

Figure 10. Airbreathing MHD accelerator concept (hypersonic mode). . . . . . . . . . 3b

Figure 11. Apollo Lightcraft in rocket or MHD-fanjet mode. . . . . . . . . . . . . . . . 3c

Figure 12. Hypersonic MHD accelerator concept (view from rear, looking forward) . . . . . 3c

Figure 13. Flight cycles for combined-cycle engines. . . . . . . . . . . . . . 4a

Figure 14. ERH thruster engine configuration. . . . . . . . . . . . . . . . 7a

Figure 15. LSD wave Lagrangian view. . . . . . . . . . . . . . . . . . . . . . . . ia

Figure 16. Cylindrical blast wave geometry. . . . . . . . . . . . . . . . . 10a

Figure 17. LSD/Blast wave expansion. . . . . . . . . . . . . . . . . . 11a

Figure 18. LSD wave impulse contributions. . . . . . . . . . . . . . . . . . . 12a

Figure 19. Supersonic air expansion over thruster plate. . . . . . . . . . . . . 15a

Figure 20. Dynamic model control volume. . . . . . . . . . . . . . . . . . . 15a

Figure 21. ERH thruster performance - Time average net thrust. . . . . . . . . . . . 16a

Figure 22. ERH thruster performance - PRF. . . . . . . . . . . . . . . . . 16a

Figure 23. ERH thruster performance - Time average power. . . . . . . . . . . . . . 17a

Figure 24. ERH thruster performance - Coupling coefficient. . . . . . . . . . . . . . 17a

Figure 25. Scramjet configuration. . . . . . . . . . . . . . . . . . 18a

Figure 26. Vehicle drag coefficient. . . . . . . . . . . . . . . . . . . . . . 20a

Figure 27. Thrust vs. Mach number for the scramjet. . . . . . . . . . . . . . . . . . 212

Figure 28. Coupling coefficient vs. Mach number for the scramjet. . . . . . . . . . . . 21b

Figure 29. Power vs. mach number for the scramjet. . . . . . . . . . . . . . 21c

Figure 30. Propulsive efficiency vs. Mach number for the scramjet. . . . . . . . . . . . 21d

Figure 31. Overall efficiency vs. Mach number for the scramjet. . . . . . . . . . . . . 2le

Figure 32. Diagram of the MHD-fanjet cycle. . . . . . . . . . . . . . . . . 22a

Figure 33. Concept for Laser-heated MHD generator. . . . . . . . . . . . . . . . . . $22 a$

Figure 34. MHD generator geometry. . . . . . . . . . . . . . . . . . . 23a

Figure 35. Distortion of the applied magnetic field in the MHD generator. . . . . . . . . 24a

Figure 36. The HIGH case MHD generator. . . . . . . . . . . . . . . . 30a

Figure 37. The LOW case MHD generator. . . . . . . . . . . . . . . . . 30b

Figure 38. MHD generator thrust. . . . . . . . . . . . . . . . . . . . . . 30c

Figure 39. MHD accelerators and generators (rear view - looking forward) . . . . . . . 31a

Figure 40. MHD accelerator and generator (side view) . . . . . . . . . . . . . 31a

Figure 41. MHD accelerator profile of $\mathrm{u}, \mathrm{T}$ and $\mathrm{P} . \ldots \ldots \ldots$

Figure 42. Electric field profile in accelerator. . . . . . . . . . . . . . . . . 32a

Figure 43. MHD-fanjet performance. . . . . . . . . . . . . . . . . . . . . 34a

Figure 44. Conductivity enhancement required in the MHD accelerator. . . . . . . . . . 34a

Figure 45. Relay Satellite configuration. . . . . . . . . . . . . . . . . 38a

Figure 46. Lightcraft drag coefficients. . . . . . . . . . . . . . . . . . 38a 


\section{LIST OP FIGURRS (cont.)}

Figure 47. Lightcraft cowl proflle. . . . . . . . . . . . . . . . . . 39a

Figure 48. Mass Breakdown for the Apollo Lightcraft. . . . . . . . . . . . . . 39a

Figure 49. Trajectory-Sensed acceleration. . . . . . . . . . . . . 43a

Figure 50. Trajectory-Dynamic pressure. . . . . . . . . . . . . . 43a

Figure 51. Trajectory-Total Laser Energy. . . . . . . . . . . . . . . . . 45a

Figure 52. Trajectory-Altitude profile. . . . . . . . . . . . . . 45b

Figure 53. Trajectory-Flight path angle. . . . . . . . . . . . 45b 


\section{ABSTRACT}

The detailed design of a beam-powered transatmospheric vehicle, "The Apollo Lightcraft ", was selected as the project for the design course. The vehicle has a lift-off gross weight of about six (6) metric tons and has the capability to transport $500 \mathrm{~kg}$ of payload (five people plus spacesuits) to low Earth orbit. Beam power was limited to 10 gigawatts. The principle goal of this project is to reduce the LEO payload delivery cost by at least three orders of magnitude below the Space Shuttle Orbiter - in the post 2020 era.

The completely reusable, single-stage-to-orbit shuttlecraft will take off and land vertically, and have a reentry heat shield integrated with its lower surface - much like the Apollo Command Module. At appropriate points along the launch trajectory, the combined-cycle propulsion system will transition through three or four airbreathing modes, and finally use a pure rocket mode for orbital insertion.

As with any revolutionary flight vehicle, engine development must proceed first. Hence, the objective for the Spring semester propulsion course was to design and perform a detailed theoretical analysis on an advanced combined-cycle engine suitable for the Apollo Lighteraft. The class determined that only three airbreathing cycles would satisfy the mission, and that the ramjet cycle is unnecessary.

The preliminary theoretical analysis of this combined- cycle engine is now complete, and the acceleration performance along representative orbital trajectories has been simulated. Average vehicle acceleration is approximately 4 - 5 G's. Transition between engine modes occurs at Mach 3, 11 and $25^{+}$. Beam power can be reduced to as low as 2.5 billion Watts without sacrificing vehicle performance. The $\mathrm{LH}_{2}$ propellant requirement is typically $300 \mathrm{~kg}$, or roughly 5 . \% of the vehicle lift-ofi weight, for delivery to a 100 nautical mile orbit. The total beam energy requirement is 520 . GW-sec for this boost mission.

Therefore, the propellant cost at current bulk $\mathrm{LH}_{2}$ rates is $\$ 975$; the energy cost is $\$ 2455$, assuming present wholesale hydroelectric power rates. The total round trip cost is only $\$ 3430$, or $\$$ 686 per person. This represents a payload delivery cost of $\$ 3.11 / \mathrm{lb}$, which is a factor of 1000 below the STS.

The Apollo Lightcraft concept is now ready for a more detailed investigation during the Fall semester "Transatmospheric Vehicle Design "course. The class will divide itself into smaller design groups (5 students in each) dedicated to: a) Aerodynamics, b) Propulsion, c) Structures, d) Thermal Analysis, e) Flight Control Systems, f) Optimal Trajectory Analysis, g) Human Factors and Life Support Systems, and h) Power-beaming Architecture These smaller desigm groups will interact with each other, the TA (a graduate student), and the instructor to finally evolve an integrated conceptual design for the Apollo Lightcraft vehicle. 


\section{CHAPTER I \\ INTRODUCTION}

Contained herein is the preliminary design and performance analysis of an advanced combined cycle engine suitable for application in a small reusable single-stage-to-orbit (SSTO) shuttlecraft. The innovative combined-cycle engine is designed to utilize highly-energetic beamed-energy sources (laser, microwave), and propell a manned transatmospheric vehicle based on the Apollo Command Module (CM).

Dubbed the "Apollo Lightcraft", this vehicle must transport a five-person crew (total payload $=500 \mathrm{Kg}$ ) into low Earth orbit (LEO) in three minutes, or anywhere on the globe in one half hour. Fully loaded for launch, the Lightcraft would weigh about six metric tons which, incidently, is equal to the dry weight of a Harrier jumpjet, Learjet, or thirty-foot long Winnebago motor home.

This study attempts to "create the blue-prints "for revolutionary globe-trotting, laser-riding shuttlecraft of the 21st century. The design group hopes that such visionary approaches will finally enable large scale access to space by reducing the payload transport costs by a factor of 1000 below the Space Shuttle Orbiter. It is with this bold objective in mind, that the present team set out to prove the feasibility of the "Apollo Lightcraft".

\section{A. Vehicle/Propulsion System Description}

Figure 1 is a sketch of a $10 \mathrm{GW}$ orbital shuttlecraft that would use advanced combined-cycle engines for propulsion. The man/machine interface is inspired by the Apollo space-capsule (see Fig. 2). It is interesting to note that this minimum weight/volume spacecraft approach was originally taken at a time when chemical rocket propulsion systems were in their infancy and rapidly maturing toward larger launch-mass (payload) capability.

As indicated in Fig. 3, the Apollo Lightcraft would have a diameter of $5 \mathrm{~m}$, gross weight at takeoff of $5550 \mathrm{Kg}$ and payload of $500 \mathrm{Kg}$. The five person crew would be propelled to orbit in a single-stage vehicle that has a reentry heatshield integrated with its entire afterbody. As portrayed in Fig. 4, the annular shroud would be translated to the full forward position, and movable thermal protection system (TPS) tiles would slide forward to close off the annular gap (i.e., between the vehicle centerbody and the shroud), just prior to reentry.

Pictured in Fig. 5 is one concept for a 10 GW Satellite Solar Power Station (SSPS), an idea first conceived by Peter Glaser of Arthur D. Little, Inc. (Cambridge, Ma.). Now imagine a hundred 


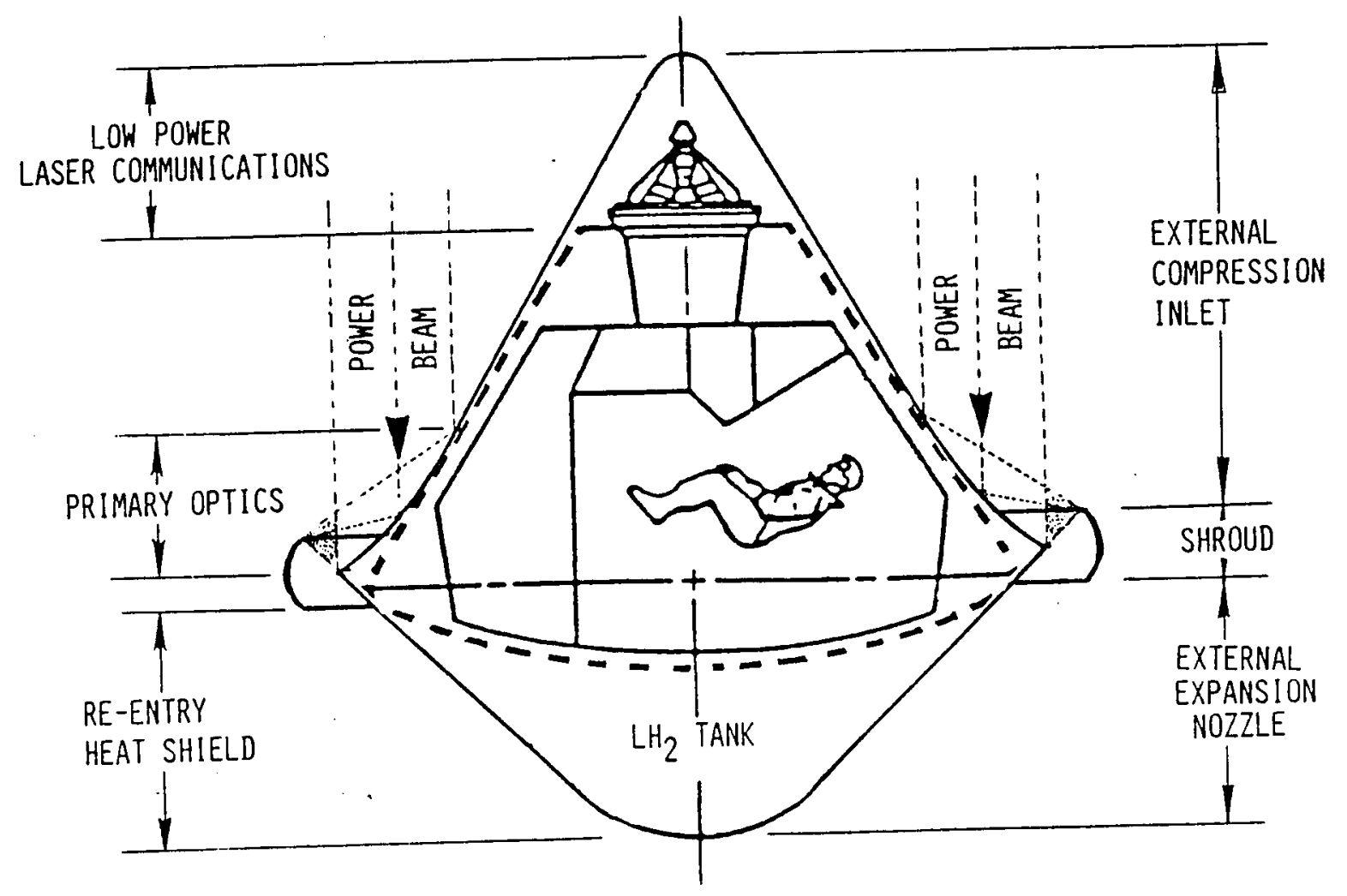

Figure 1. The Apollo Lightcraft configuration (10-GW machine).

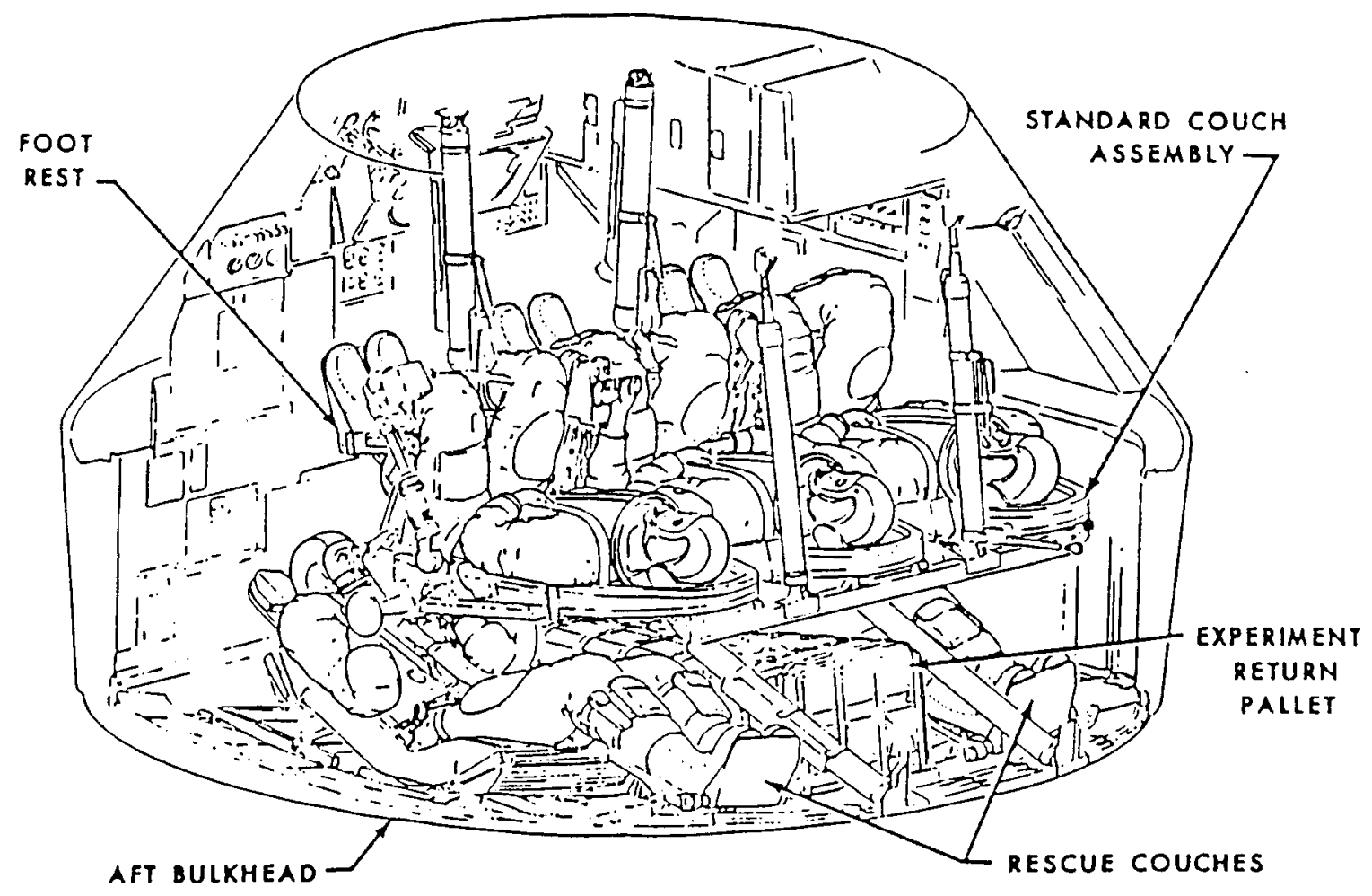

Figure 2. Interior of the Apollo Command Module. 


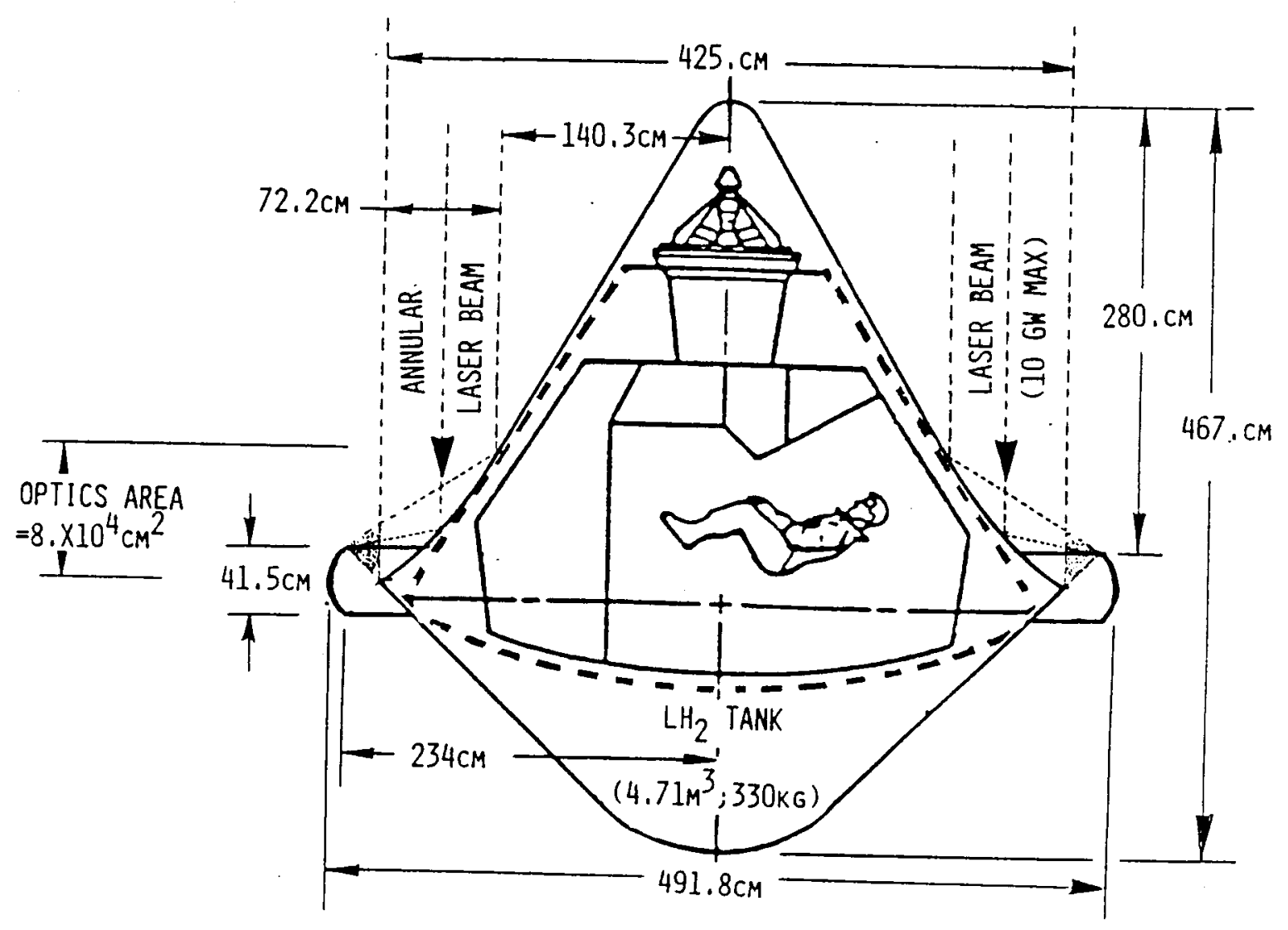

Figure 3. Dimensions for the Apollo Lightcraft.

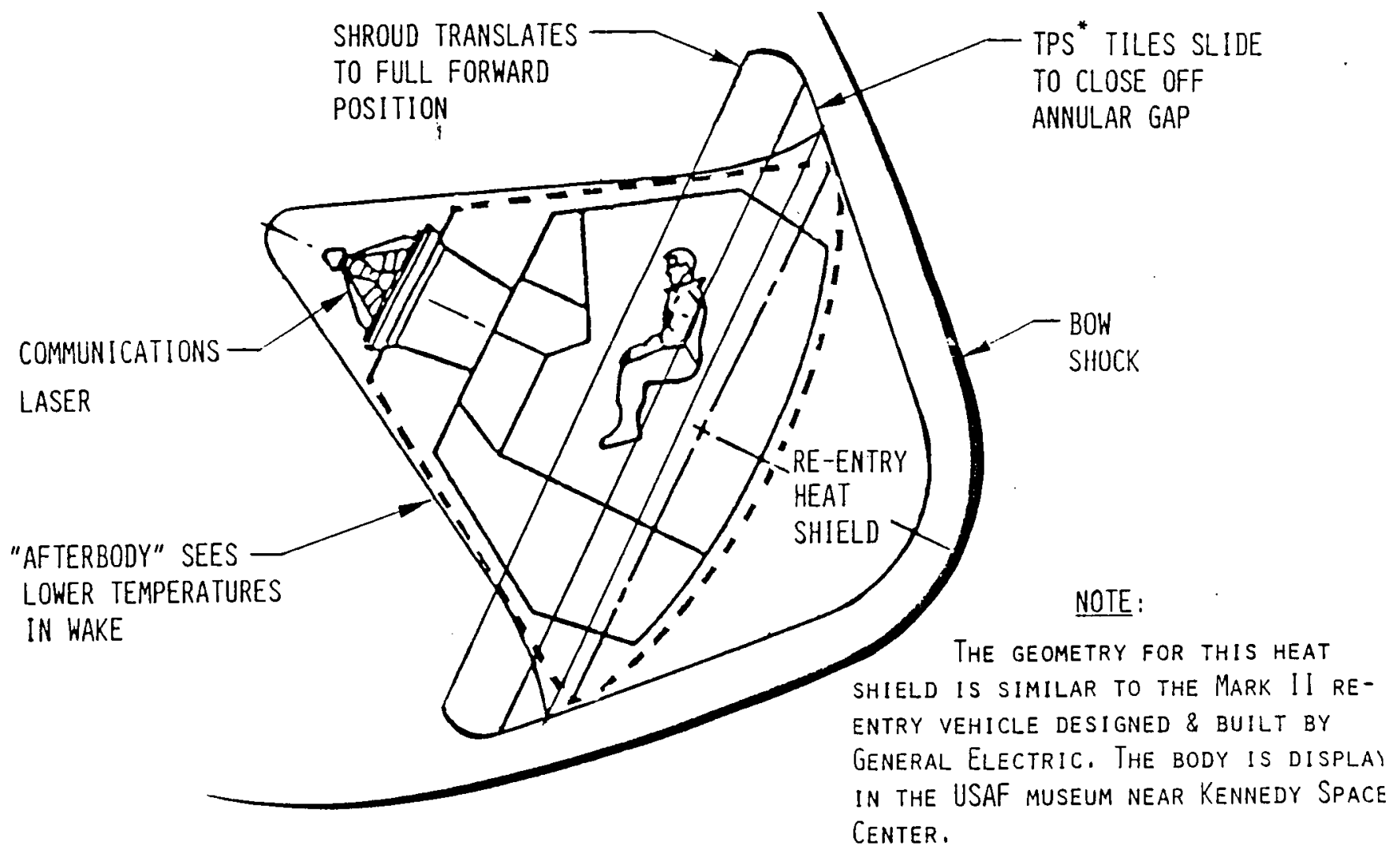

Figure 4. Reentry configuration for the Apollo Lightcraft. 
of these SSPS's linked together into a space-based "power grid "in which "wireless "transmission of energy is accomplished by laser (or microwave) beams - sometime in the early 21st century. The succesful mating of advanced beam-powered flight vehicles (such as the Apollo Lightcraft) to highly reliable lasers and satellite solar power stations would revolutionize air and space transport as we know it today. Clearly, all necessary safeguards must be detigned into the space power system in order to provide extreme levels of network security, and accountability for every Joule of energy beamed throughout cislunar space.

As indicated in Fig. 3, beam power is first received by the Apollo Lightcraft across a 4.25 meter diameter centerbody primary optics at an intensity below that which would ignite a high temperature plasma (e.g., $1-2 x 10^{4} \mathrm{~W} / \mathrm{cm}^{2}$ for 10.6 micron light). This plasma-ignition threshold is lower for longer wavelengths, and higher for shorter wavelengths. Hence, the greatest power is delivered at the ultraviolet limit of about $0.35 \mu \mathrm{m}$ - beyond which atmospheric scattering dominates. The primary optic surface area of $8 \times 10^{4} \mathrm{~cm}^{2}$ (see Fig. 3) would permit the reception of 0.8 to 1.6 GW of continuous laser power at $10.6 \mu \mathrm{m}$. At the ultraviolet limit, this value can be increased by a factor of five or so. It should be noted, however, that the first two engine modes for the Apollo Lightcraft are pulsed, and one must be careful to reduce this peak continuous power level by the effective duty cycle.

The Apollo Lightcraft combined-cycle engine would permit vertical takeoffs and (powered) landings. While accelerating to orbit this engine would "shift gears ", much like a 4 or 5 speed high performance sports car. The advanced engine would have three or four airbreathing modes, plus a rocket mode used for orbit insertion. These engine cycles (or modes) are chosen to maximize flight performance throughout a given range of Mach numbers, with distinctly identified transition points. It is evident that the choice of specific combined-cycle engine schemes has a large effect upon the overall vehicle configuration.

For the Apollo Lightitcraft, the most logical choice for the combined-cycle engine is as follows:

Mode 1 - ERH thruster;

Mode 2- ramjet (optional);

Mode 3- scramjet;

Mode 4- MHD - fanjet; and,

Mode 5 - rocket. All engine modes are powered by beamed energy.

Fig. 5 portrays the Apollo Llghtcraft in the External-Radiation- Heated (ERH) thruster mode, which is used for powered vertical takeoffs and landings, as well as acceleration runs up to Mach 3. Note that the ERH thruster reaction surface (see Fig. 6) is easily combined with a reentry heat shield - with little additional weight penalty. 


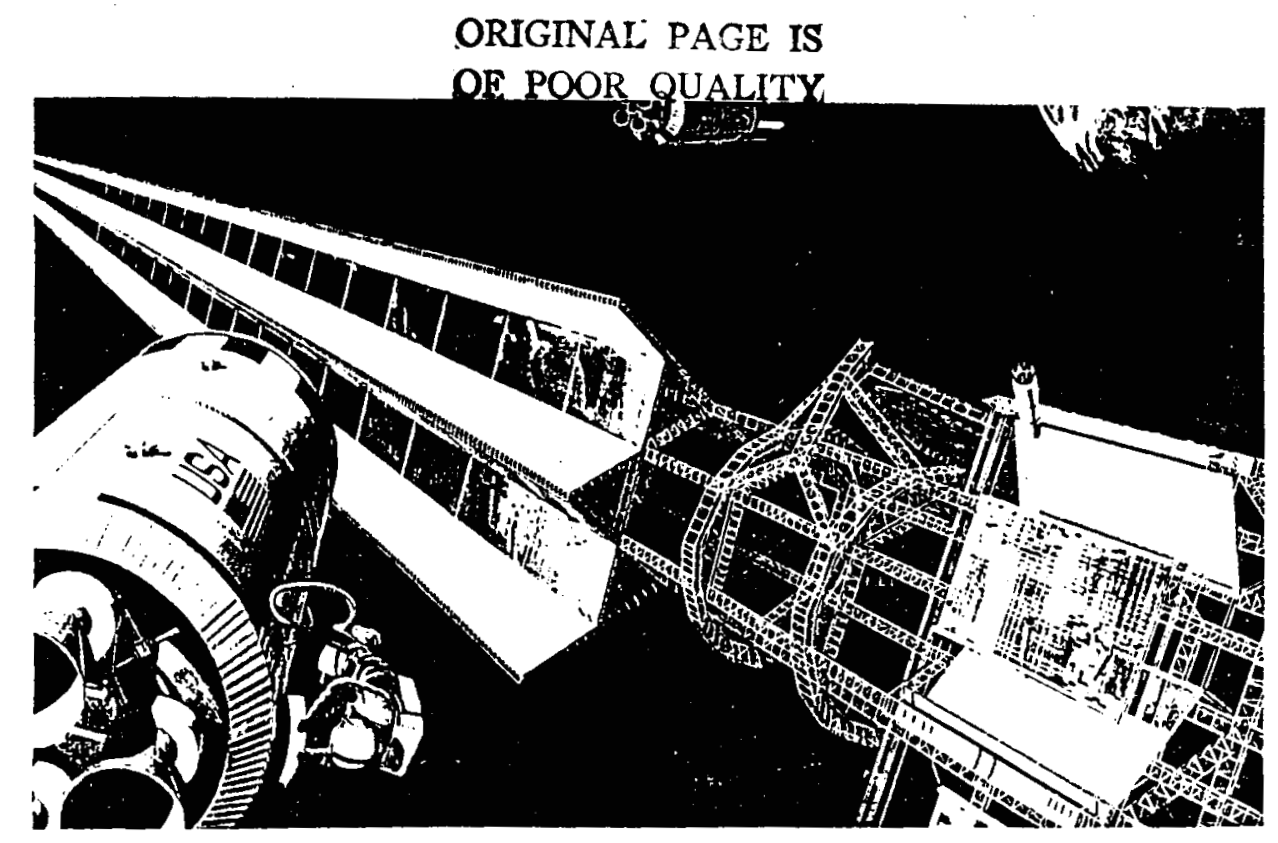

Figure 5. $10 \mathrm{GW}$ Satellite Solar Power Station.

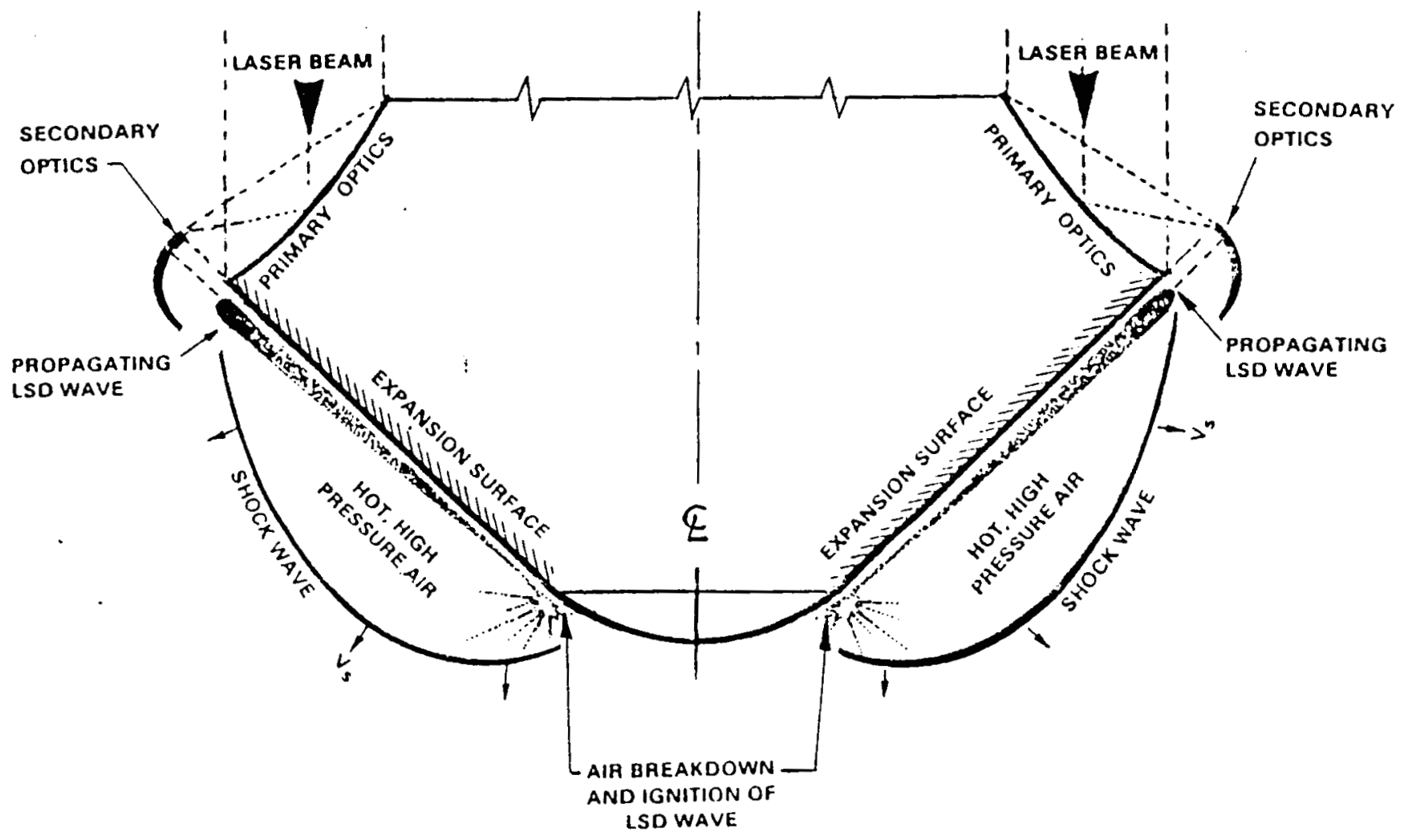

Figure 6. Apollo Lightcraft in ERH thruster mode. 
It has been discovered that a "pop-up "maneuver (using the ERH thruster) must be utilized before starting the acceleration run to orbit. Under a low power microwave beam link, the shuttlecraft could climb vertically through any weather (clouds, fog, rain, snow, etc.), until an altitude of 20 - $40 \mathrm{kft}$. is reached. Then the Lightcraft would rotate to engage high power laser beam link from a low altitude relay station (e.8., $185 \mathrm{~km}$ ), and start accelerating. Not only does this procedure guarantee maximum acceleration performance, but it also insures all-weather capability.

Upon reaching Mach 3, propulsive power would be transferred to the ramjet (or perhaps directly to the scramjet) mode. The incoming laser power would be absorbed within the annular shroud region. An annular "planar heater" (pictured in Fig. 7) would be created by repetitively pulsed LSD waves that propagate at right angles across the duct flow. Note that the planar heater would promote turbulent mixing of the internal flow and enable an exceptionally short shroud.

As the vehicle continues to accelerate, this duct flow would increase to supersonic velocity, and the engine would automatically transition to the scramjet mode. As indicated in Fig. 8, the annular shroud would translate aft with increasing flight Mach number such that the conical bow shock is always attached at the shroud forward lip (i.e., beyond Mach 3, the design Mach number of the inlet).

At some point beyond Mach 11, frozen flow losses would dominate, and net thrust would fall to zero. The Apollo Lightcraft engine would then transition to the MHD-fanjet propulsion cycle portrayed in Fig. 9. As indicated, laser-heated $H_{2}$ rocket-gas generators would extract 40 to $50 \%$ of the enthalpy as electric power and deliver it to an airbreathing "electric fan " (see Fig. 10).

The Apollo Lightcraft annular duct would be converted to an MHD air accelerator (see Fig. 11) simply by énergizing a series of cryogenic or superconducting electromagnets. As shown in Fig. 12, these electromagnets are integrated within the shroud support struts and generate a confined toroidal magnetic field. The magnetic field configuration is designed to close upon itself, such that the field lines do not arc out into the surrounding airspace external to the engine. It is important to note that the MHD fanjet can exhibit coupling coefflcients in the same range as a pure laser-heated rocket (i.e., 50 - $100 \mathrm{~N} / \mathrm{MW}$ ), but can have specific impulses an order of moonitude higher (e.g., 10,000 to $20,000 \mathrm{sec}$, vs. 1000 to $2000 \mathrm{sec}$.).

Once orbital velocity has been reached at an altitude between 200 to $250 \mathrm{kft}$., the MHDfanjet cycle would be transitioned to pure rocket mode (i.e., electromagnets are shut off), and the vehicle would pitch up to leave the atmosphere. The twelve short laser heated rocket exhaust nozzles are positioned radially about the vehicle afterbody - which acts as a plug nozzle - to secure a large expansion ratio at high altitudes. The mass flow rate of hydrogen would roughly double to $11 . \mathrm{Kg} / \mathrm{s}$, and the stagnation temperature would fall to perhaps $8000 \mathrm{~K}$, with the stagnation pressure reduced 
NITES:

- "Planar heater" promutes

tURBULENT MIXING AND

ENABLES EXCEPTIONALLY

SHURT SHROUD.

\# PLANAR HEATER IS ACTIVATED BY RP LSD-WAVES.

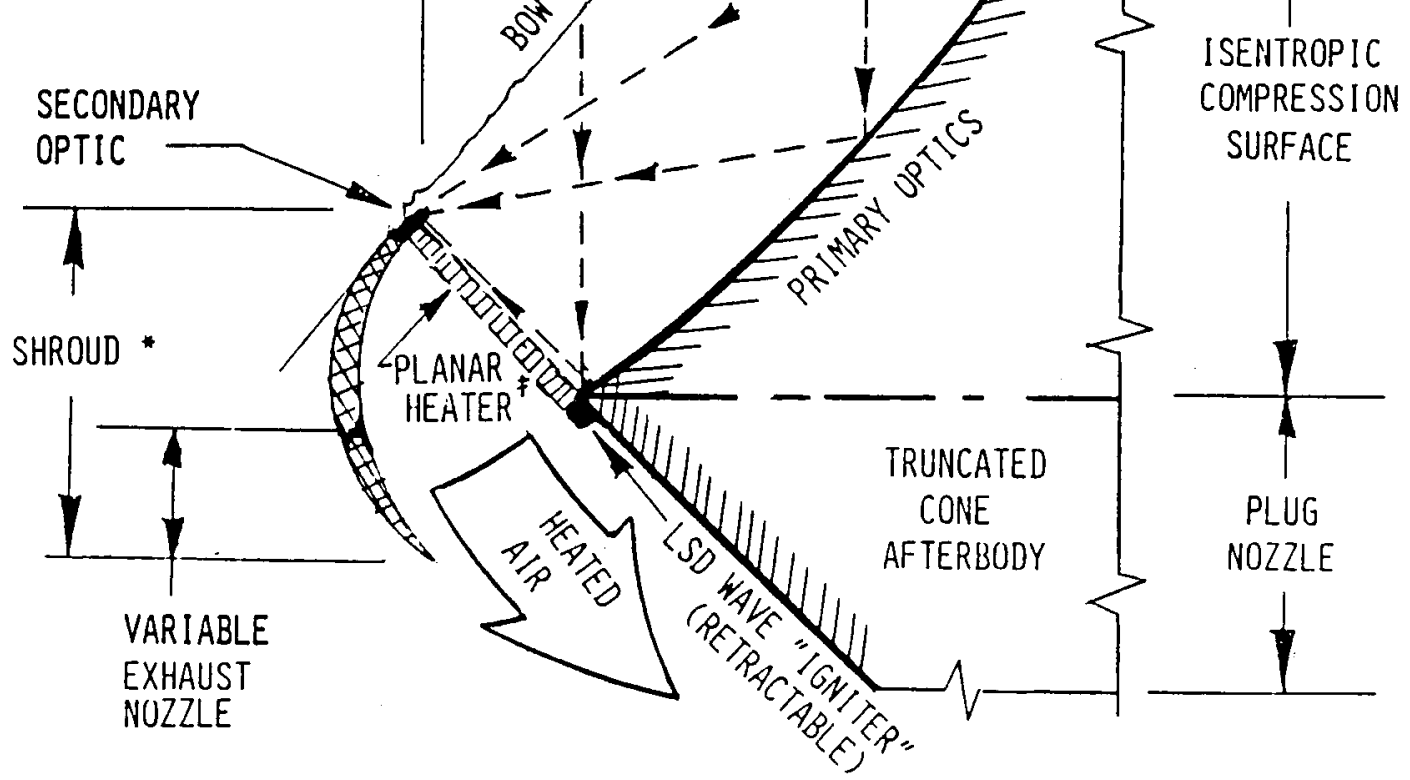

Figure 7. Repetitively-pulsed "planar heater " for ramjet and scramjet modes.

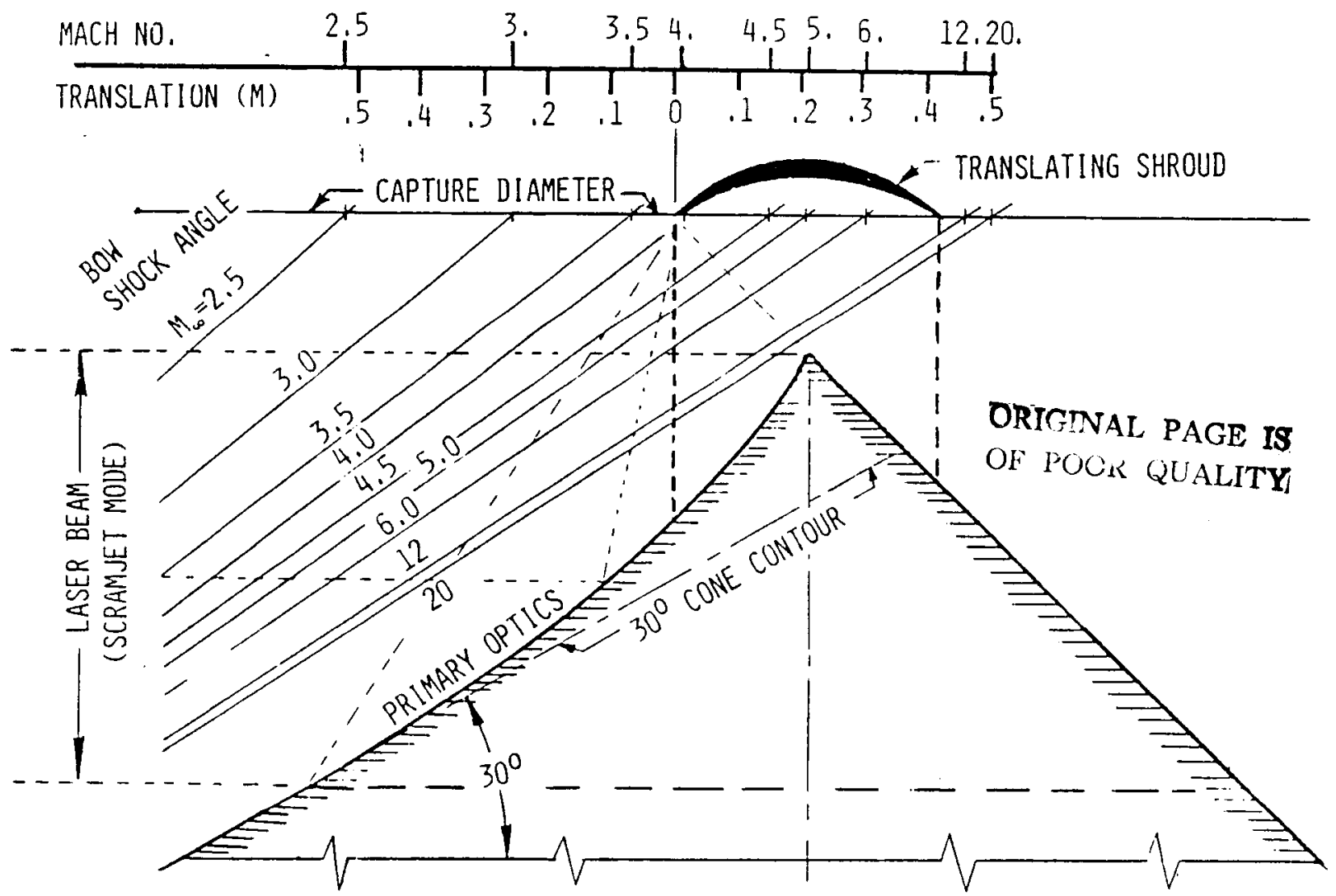

Figure 8. Bow shock angle vs. shroud translation. 


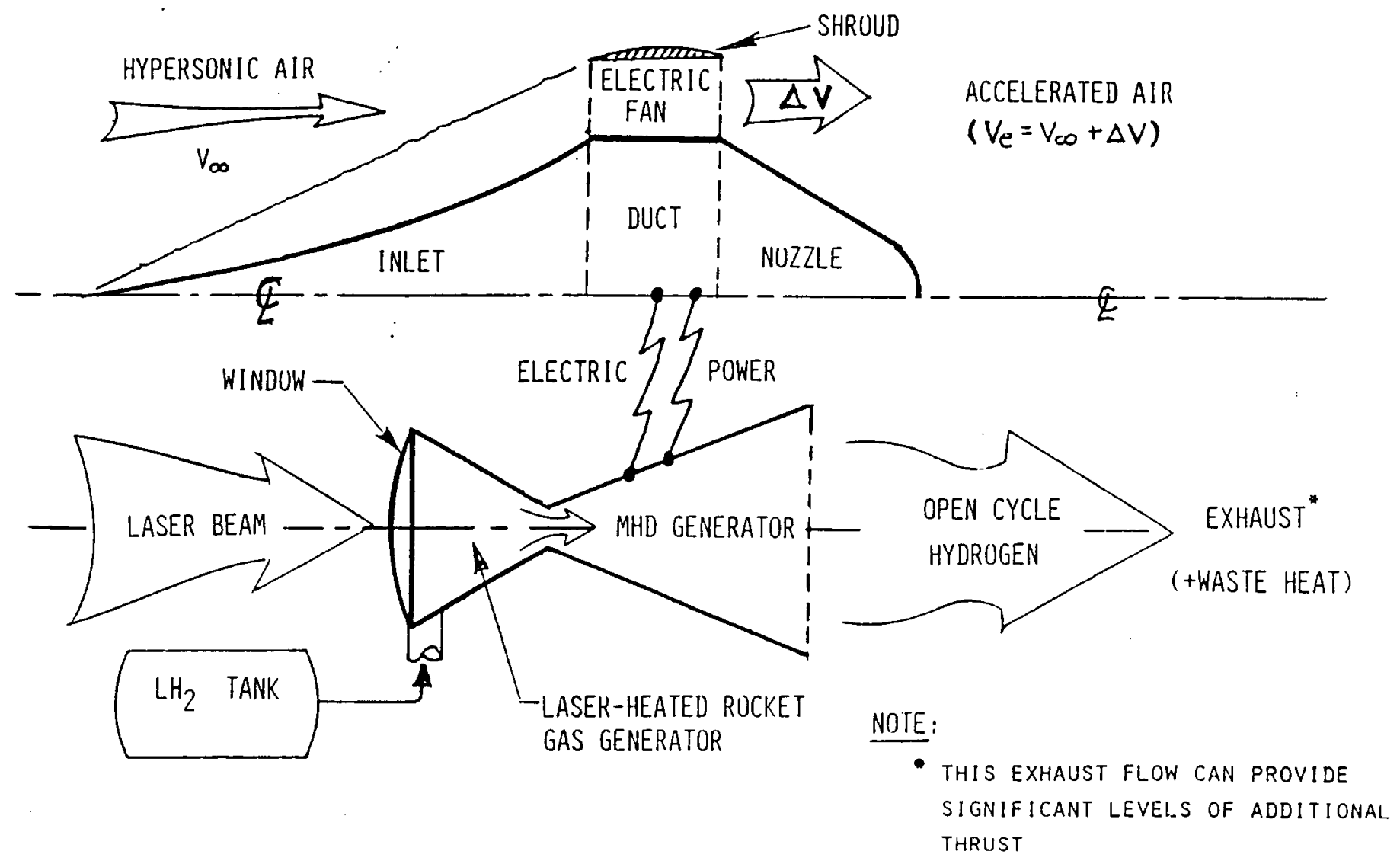

Figure 9. Diagram of MHD-fanjet cycle (an electric air-turborocket).

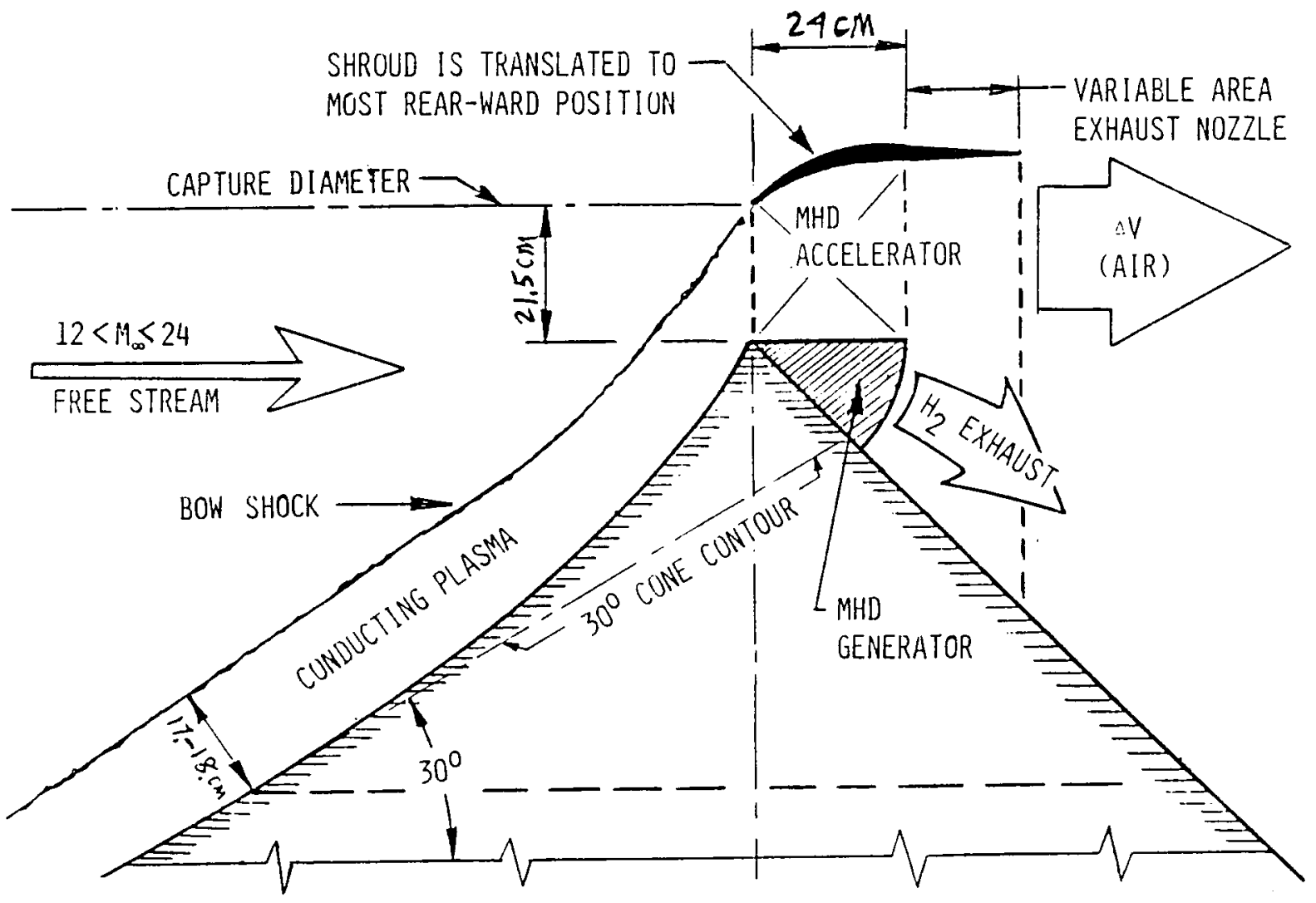

Figure 10. Airbreathing MHD accelerator concept (hypersonic mode). 


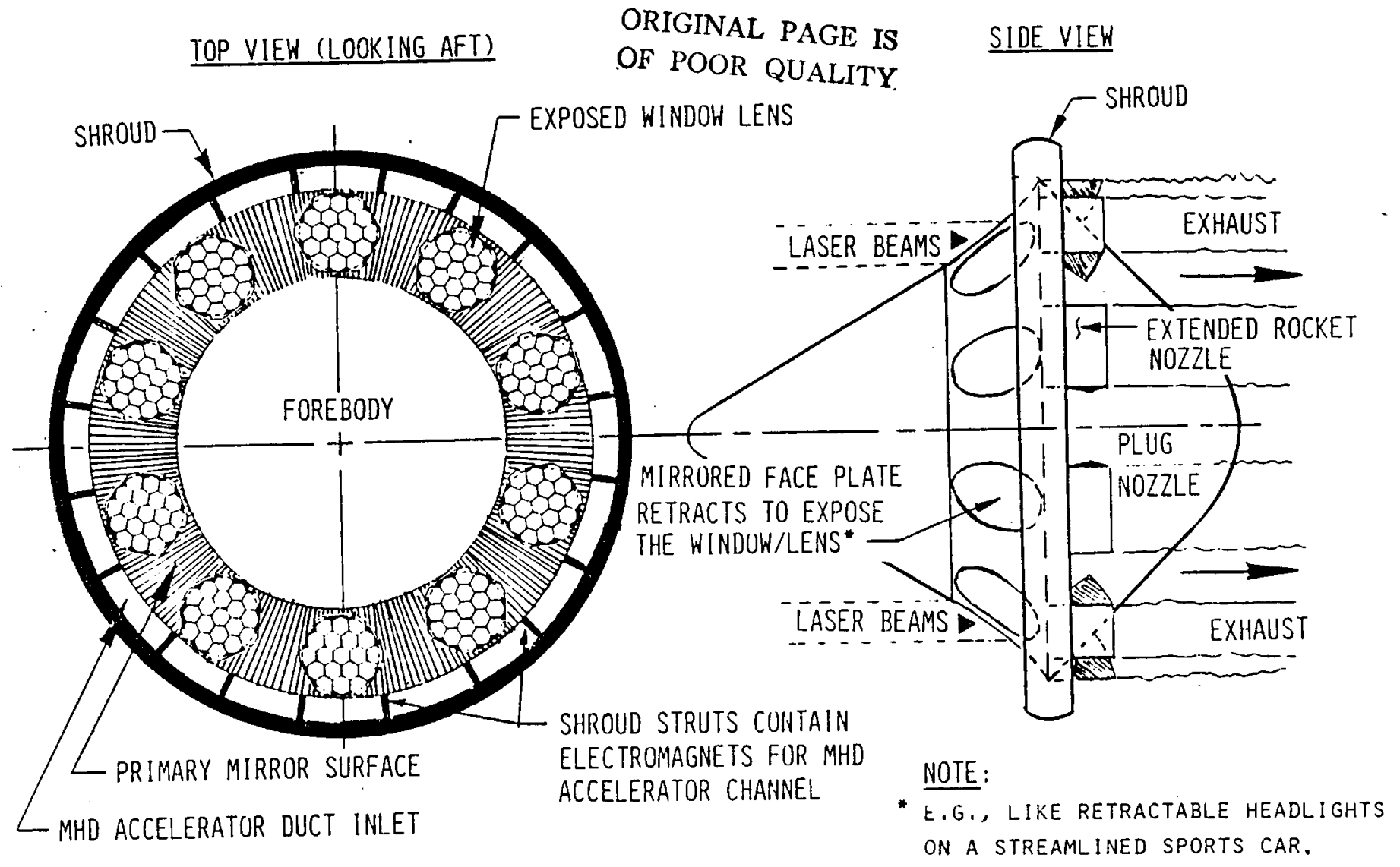

Figure 11. Apollo Lightcraft in rocket or MHD-fanjet mode.

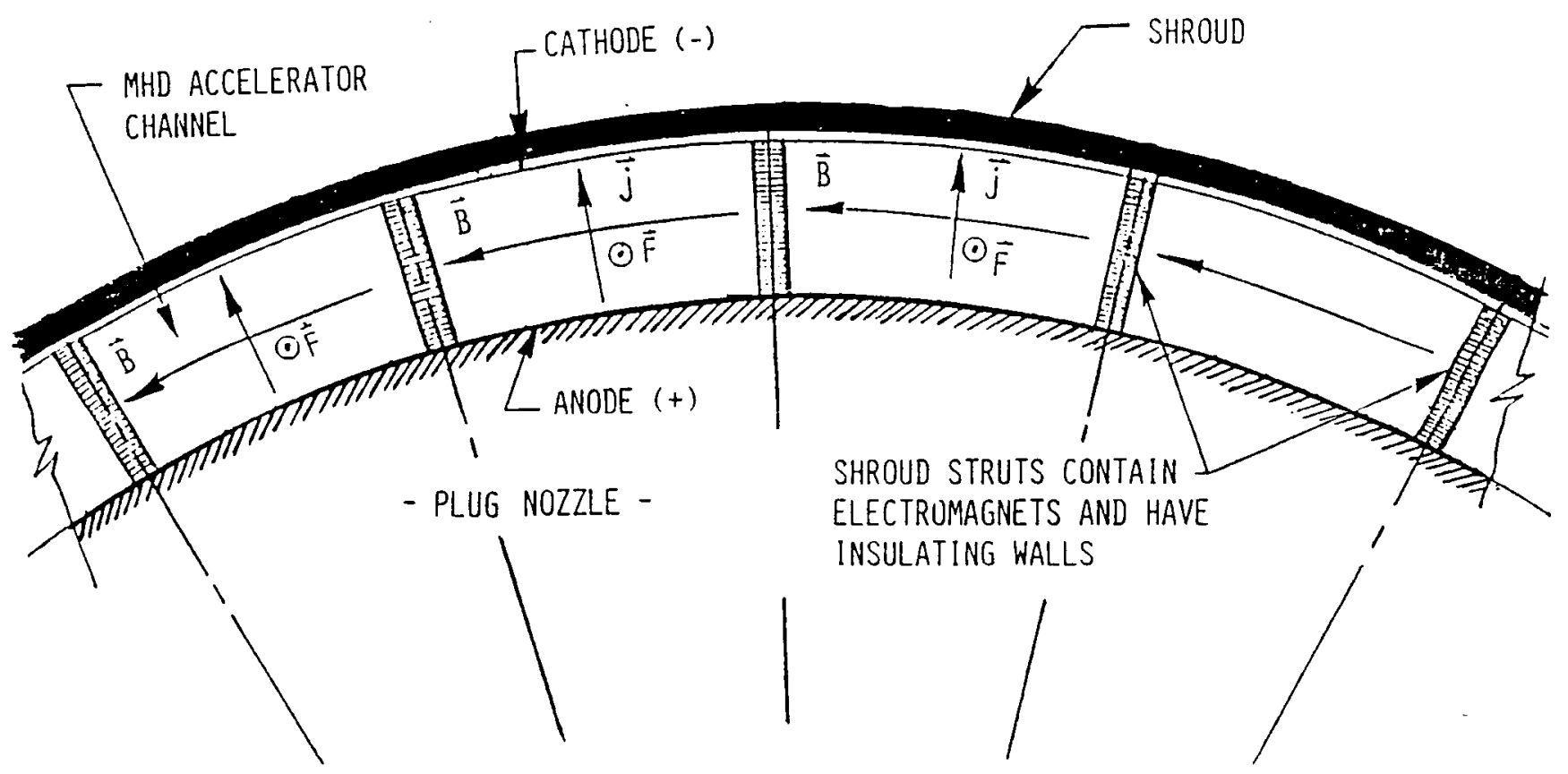

Figure 12. Hypersonic MHD accelerator concept (view from rear, looking forward). 
to 60 bars. Under these conditions, the rockets would develop a specific impulse of 2000 to 2400 s. With a thermal efficiency of $66 \%$, the engine could produce a coupling coefficient of $67 \mathrm{~N} / \mathrm{MW}$. At a vehicle mass of $5550 \mathrm{Kg}$, this gross thrust level of $2.35 \times 10^{5} \mathrm{~N}$ would result in a maximum acceleration of $4.3 \mathrm{~g}$ (i.e., if vehicle drag is neglected). Since the vehicle would have depleted some fraction (e.g., one-third), of the onboard propellant by this time, the acceleration could be a little higher. If a lower acceleration is desired, it could be exchanged for a higher $I_{o} p$ from these rocket engines.

Fig. 13 attempts to summarize in one chart the coupling coefficients anticipated from laserheated rockets, in comparison with most known airbreathing engine cycles. The goal of advanced trans-atmospheric "combined-cycle" engines is, of course, to reduce the fuel fraction required to carry a given payload into orbit. Indications are that laser-powered, SSTO combined-cycle engines could enable fuel fractions as low as 5 to $10 \%$. Saying it another way, mass fractions (initial/final) of 1.11 or less may be altogether feasible!

Once the decision is made to leave low Earth orbit, the Apollo Lightcraft rocket engines would be fired (briefly) to slow the vehicle by perhaps $100 \mathrm{~m} / \mathrm{sec}$. The spacecraft would then reenter the atmosphere, shielded by a thermal protection system similar in design to that used on the earlier Apollo capsule. Eventually the Lightcraft would deccelerate to a specific subsonic terminal velocity (i.e., a free-fall), and just prior to landing, the ERH thruster would be fired for a few seconds to provide a braking force. The long-term goal for such advanced laser-powered shuttlecraft should be the same kind of operational flexibility demonstrated by today's airlines, or better.

Since Lightcraft will have VTOL capability, they could in principle be set down almost anywhere. They do not require a 10,000 foot long runway; just a helicopter pad will do. Also, since the power beam provides the energy for propulsion, the $\mathrm{LH}_{2}$ propellant (used during the high performance MHD-Fanjet and rocket modes) might possibly be replaced by something as simple as water Although the acceleration performance and specific impulse would suffer, the cost of deionized water is negligible relative to $\mathrm{LH}_{2}$. Hence the overall payload delivery cost might be roughly the same - and you could "fill'er up" at your house with the garden hosel Finally, judging on the basis of the available tankage volume in the Apollo Lightcraft, the total "fuel load "might account for 6 to $46 \%$ of the launch weight, depending on the performance of the combined-cycle engine scheme and the choice of propellant (ie., $\mathrm{LH}_{2}$ vs. $\mathrm{H}_{2} \mathrm{O}$, respectively).

In some post 2020 era, space travel will be commonplace, and the present large "standing army " ground crew will be replaced by efficient macro-computers - which schedule launch windows, effortlessly deliver beam power from the Space Power Grid to hundreds of spacecraft simultaneously, and electronically mail out the end-of-the-month billings to millions of satisfied "jet-setters ". The 


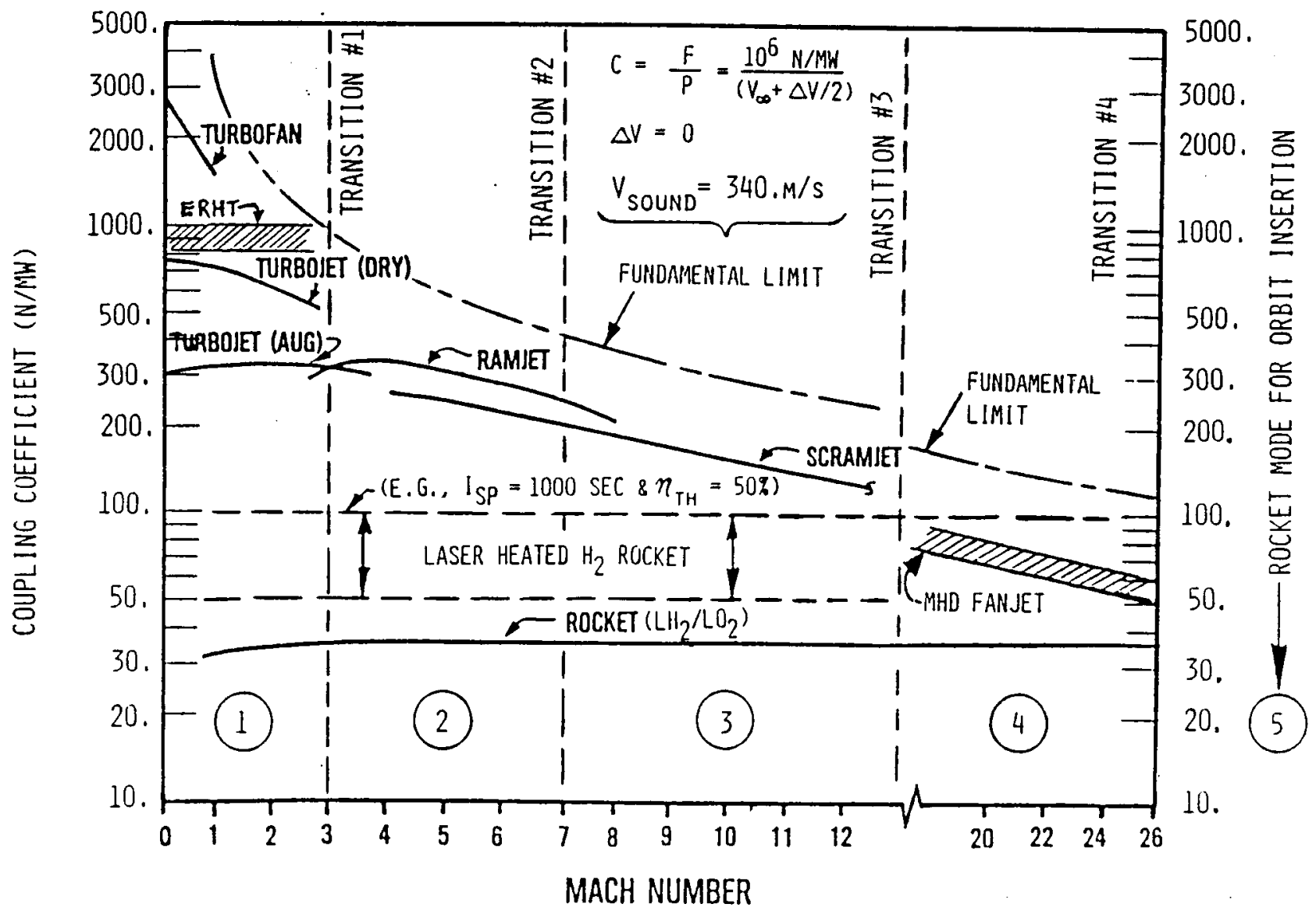

Figure 13. Flight cycles for combined-cycle engines. 
dominant costs will be for propellant and power. Payload delivery coato will eventually plunge a factor of 1000 below that of the Space Shuttle Orbiter.

\section{B. Design Study and Final Report Orgmaisation}

The Apollo Lightcraft combined-cycle engine study was carried out as a "proof-of-concept " investigation, and consisted of the following eight tasks:

Task 1 : Compute Maximum Available Beam Power vs. Wavelength

Task 2 : Select Engine Modes and Transition Points

Task 3 : Develop Detailed Analytical Models for Engine Performance

Task 4 : Conceive Integrated Engine/Vehicle/Optics Configuration

Task 5 : Determine Forebody, Shroud and Base Drag vs. Mach No. and Altitude

Task 6 : Analyze Altitude vs. Mach Number Performance of all Engine Modes

Task 7 : Estimate Shuttlecraft Mass Breakdown

Task 8 : Predict Shuttlecraft Performance Capability Along Orbital Trajectory

Principal results of the first six tasks are reported in Chapts. II through $\mathrm{V}$ of the present report. Chapter II presents the model for the ERH thruster; chapter III, the Ramjet/Scramjet model; chapter IV, the MHD-Fanjet model, and finally chapter V, the rocket model.

These detailed analytical models were developed for assessing the thermodynamics and gasdynamics of all four airbreathing engine modes given specific data on component technology. The models were assembled to facilitate the computation of thrust, coupling coefficient, specific impulse $\left(I_{\bullet} p\right)$, and beam power - as a function of flight conditions (i.e., Mach number and altitude). These models permit the computation of gas stagnation and static conditions, cross-sectional flow dimensions and flow Mach number at all important engine stations. Complete engine diagrams showing the important engine stations are presented with the performance in later chapters of this report.

The importance of inlet centerbody cone angle, shroud configuration and plug nozzle design upon engine total pressure recovery were found to be significant. The choice of inlet cone angle, for example, is a compromise between the needs of two separate engine modes: the MHD-fanjet benefits from a blunt cone inlet to enhance flow ionization; whereas, the ramjet and scramjet would like sharp cone inlets for maximum total pressure recovery. Note also that light-weight structural design dictates minimal enclosed volume, which points to the blunt cone solution.

Using the detailed analytical models developed in Task 3 and the data on specific engine components, the altitude/velocity performance was predicted for the airbreathing comined-cycle 
engine. Partial (throttled) and full thrust in each mode were characterized. Optimum performance in all modes was determined, as well as the most favorable transition Mach number between modes. Detailed computer models were used to carry out these calculations. Principal results included graphical plots of thrust, specific impulse, coupling coefficient, propulsive efficiency and beam power vs. flight Mach number - with altitude as the parametric variable.

The results of Task 8, which predicted the performance capability of the Apollo Lightcraft, are presented in chapter VI. In addition to determining the acceleration schedule (i.e., G-level vs. time) and the "time-to-climb" to orbit, a primary output of this task was to predict the overall vehicle mass ratio (i.e., final-to-initial weight), time-average beam power along the trajectory, and total boost energy in gigawatt-seconds required to deliver the $500 \mathrm{Kg}$ payload to orbit. The group assumed a beamed energy cost of 1.7 cents/KW-hr (current NE Canadian wholesale hydro-power rate), and a price of $\$ 3.252 / \mathrm{Kg}$ for $L H_{2}$ (current Shuttle Orbiter rate).

Finally, chapter VII summarizes the principal conclusions reached in this initial proof-ofconcept study on the Apollo Lightcraft combined-cycle engine. 


\section{CHAPTER II ERH THRUSTSR}

The External Radiation Heated (ERH) thruster, as its name implies, is an external airbreathing engine which utilizes a high intensity laser beam to produce thrust. The thruster itself is extremely simple, consisting only of an impulse surface, which is integrated in the aft section of a flight platform and able to withstand high temperatures and pressures. In the present analysis, the ERH thruster surface is a circular frustum which makes up the aft section of the Apollo Lightcraft, see Figure 14. To generate thrust, a series of laser-induced cylindrical blast waves are initiated adjacent to the thruster surface; as these blast waves expand, impulse is delivered to this surface. To understand the principles of this thruster, it is first instructive to examine the laser-induced blast wave phenomenon which is fundamental to its operation.

\section{A. BASIC OPERATING PRINCIPLES}

When a high intensity laser beam is focused, a detonation wave forms: the structure of which consists of a shock followed by an absorption zone. Within the absorption zone, laser energy is transferred into the fluid, in the form of thermal energy, by the mechanism of inverse bremsstrahlung. This Laser-Supported Detonation (LSD) wave propagates up the laser beam at supersonic velocities and leaves behind a high energy, high pressure plasma zone. This, in turn, expands into the ambient air much like a blast wave generated by a conventional chemical explosion. Because LSD waves propagate toward the laser source much faster than the radial expansion of the hot plasma behind the detonation front, the resulting blast wave is cylindrical in shape.

The ERH thruster utilizing LSD blast waves has the following operation. First, several laser beams projected parallel to and across the thruster surface, are focused to initiate LSD waves. As these LSD waves propagate across the thruster plate, high pressure cylindrical blast waves are formed and subsequently expand. The portion of the blast wave in contact with the thruster surface exerts a pressure force upon the surface. It is this impulse loading that results in thrust. Once the Blast wave pressure decays to the local ambient pressure, the thruster surface must be cleared of the hot plasma and replaced with ambient air before another set of LSD waves can be initiated. This exchange of the hot plasma with cool, unprocessed air is equivalent to the heat rejection portion of a conventional thermodynamic cycle. In the present analysis, this exchange process is referred to as surface refresh.

From the above discussion, it is evident that the ERH thruster is a repetitively-pulsed 


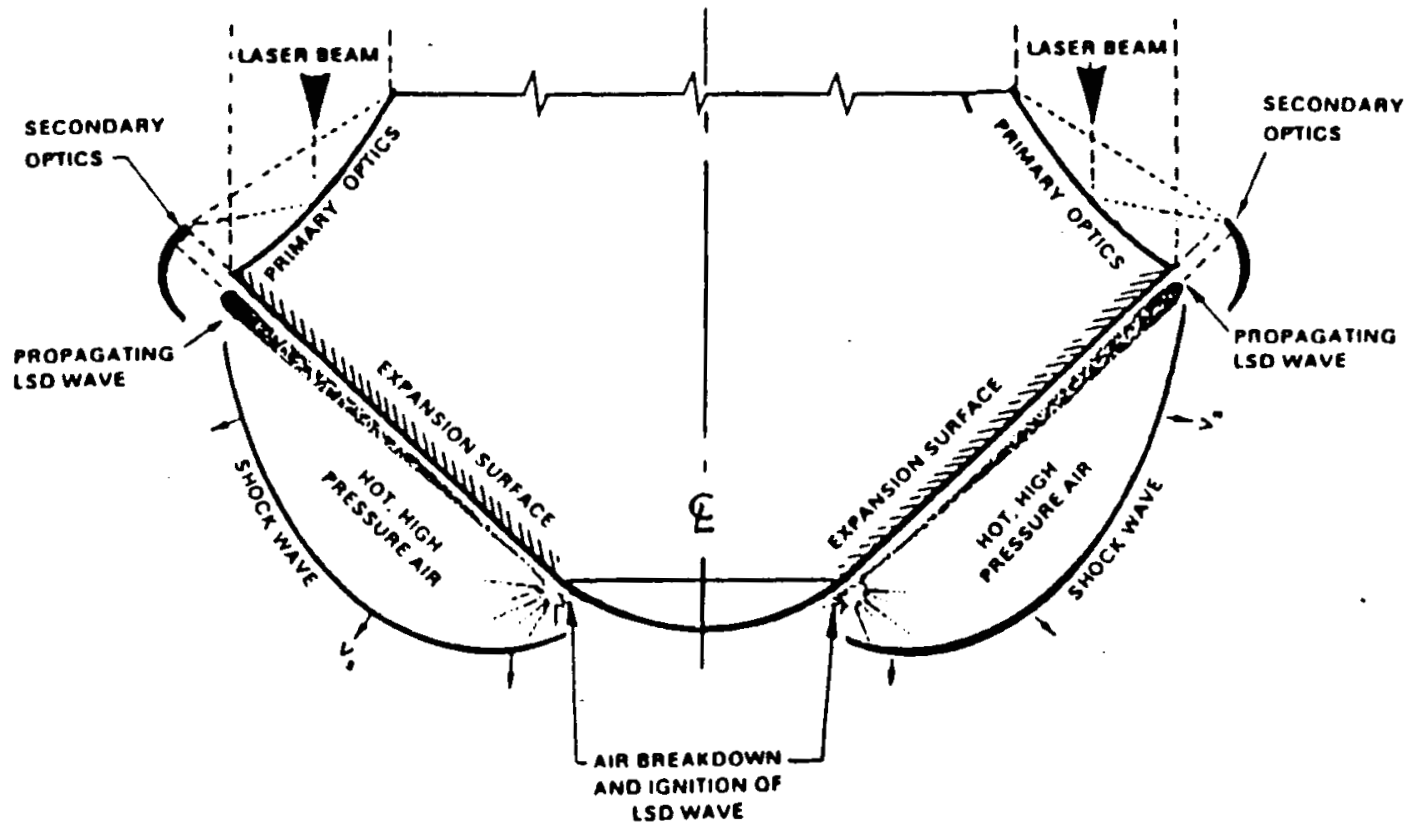

Figure 14. ERH thruster engine configuration.

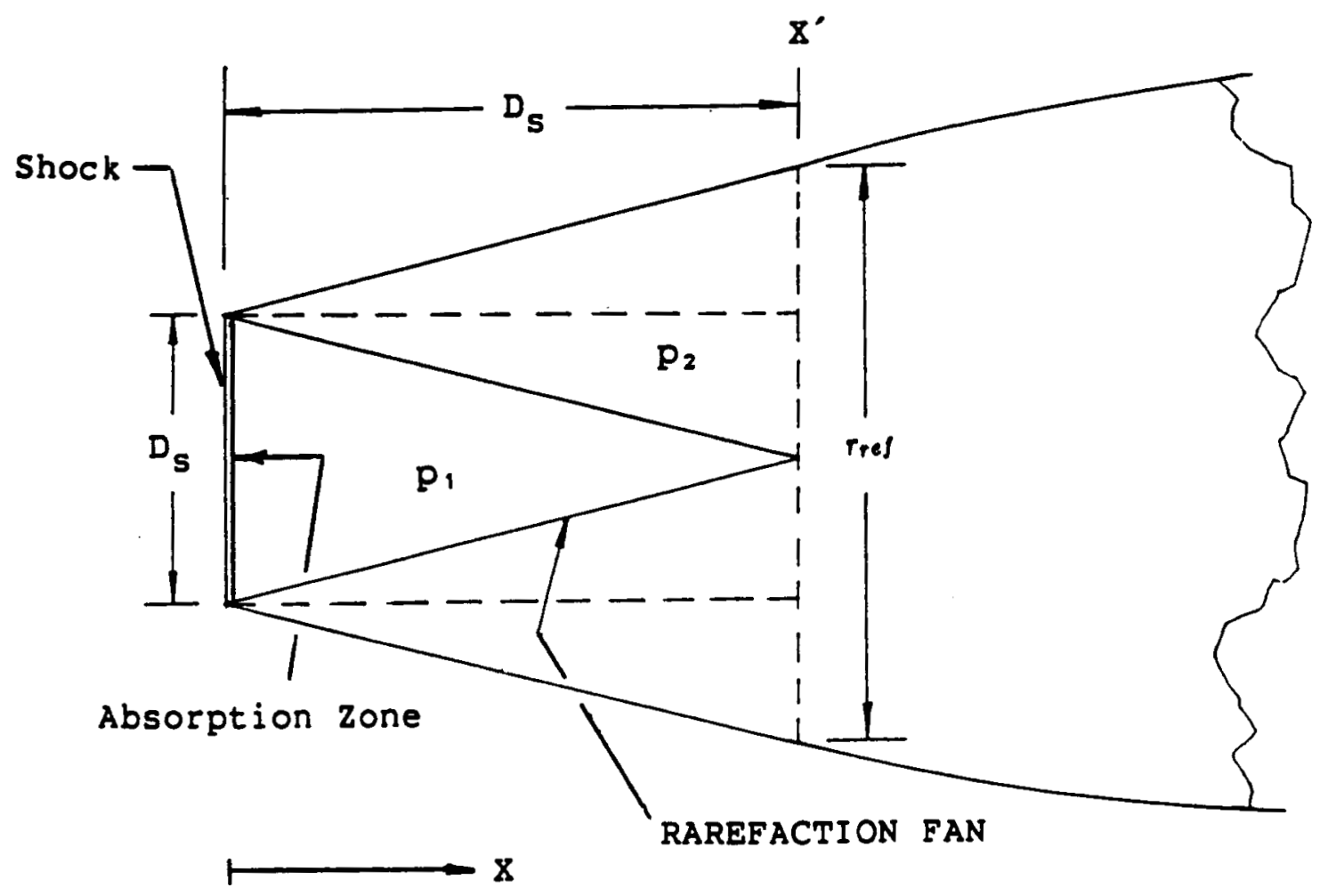

Figure 15. LSD wave Lagrangian view. 
engine, in which its Pulse Repetition Frequency (PRF) is governed essentially by the blast wave decay time and the thruster surface refresh time; the total cycle time is dependent on the local ambient air pressure and density. This implies that thruster performance varies with altitude and flight velocity. Before the ERH thruster performance is analyzed, the structure of the LSD wave is investigated and a static model for the ERH thruster is developed.

\section{B. LSD BLAST WAVT ANALYSIS}

A laser supported detonation (LSD) wavecan be analyzed as if it were a chemical detonation; the chemical heat of reaction is replaced by the absorbed laser heat flux. Therefore, the ZND detonation wave model, developed by Zel'dovich, Von Newman, and Döring for chemical detonations, can be adapted and used to analyze the LSD wavestructure. In the ZND model, chemical detonation wave structure is modeled as a one-dimensional shock front followed by a high speed deflagation; for LSD waves the deflagration is replaced by a thin absorption zone. In LSD waves there is a coupling between the fluid dynamics and the absorption kinetics. The leading shock ionizes the air thereby allowing the formation of the absorption zone, and likewise, the laser energy absorbed within this zone drives the shock. Because of this coupling, the detonation wave structure occurs only when laser intensities are above $10^{6}-10^{7} W / C M^{2}$ (for $10.6 \mu \mathrm{m}$ radiation). Raizer[13], using the ZND wave structure, was able to derive the following wave velocity expression for a LSD wave propagating into quiescent gas.

$$
V_{D}=\left[2\left(\gamma^{2}-1\right) \frac{\Phi}{\rho_{0}}\right]^{1 / 9}
$$

Up to this point, the detonation wave has been viewed from an Eulerian frame of reference It is, however, generally easier to examine the detonation wave structure from a Lagrangian frame. In the Lagrangian frame, the observer is assumed to be traveling on the shock front, typically referred to as shock coordinates. To convert from Eulerian coordinates to shock coordinates the following transformation is applied:

$$
u=u^{\prime}-V_{D}
$$

In Fig. 15 the structure of the LSD wave is shown in shock coordinates. Observe that a radial rarefaction fan begins to propagate inward toward the blast wave center directly behind the absorption zone. Also note, that regions of steady pressure and expansion velocity exist ahead and behind the rarefaction fan. In an actual blast wave, the converging expansion fan is reflected at wave's centerline and forms a second rarefaction wave which propagates outward from the wave's center. 
In this analyeis, only the firet rarefaction wave will be of interent. Once the firet fan reaches the centerline, at location $X^{\prime}$ in Figure 15, the blast wave decay is approximated using self-similar theory. Previous investigators $[7,12]$ have sucessfully applied self-similar blast wave theory away from the LSD detonation front. Here, the blast wave will be analyzed in two sections: flrst, using the method of characteristics in the region between the absorption front and the plane where the first fan reaches the center line; and thereafter, using self-similar blast wave theory.

In this model, axial relaxation of the LSD blast wave is neglected, and radial expansion is assumed to dominate the pressure decay process. Also, the axial velocity is assumed small when viewed from the Eulerian reference frame. These assumptions permit the use of the following onedimensional unsteady flow equations to describe the blast wave expansion:

$$
\begin{gathered}
\frac{\partial \rho}{\partial t}+u \frac{\partial \rho}{\partial r}+\rho \frac{\partial u}{\partial r}+\frac{\rho u}{r}=0 \\
\rho\left(\frac{\partial u}{\partial t}+u \frac{\partial u}{\partial r}\right)+\frac{\partial P}{\partial r}=0 \\
\frac{\partial S}{\partial t}+u \frac{\partial S}{\partial r}=0
\end{gathered}
$$

For the present development, the last term of the continuity equation is dropped. Using an order of magnitude analysis, it can be shown, that the error introduced by neglecting this terms is small; its removal simplifies the development of the characteristic equations. Using the method of characteristics, the above system of partial differential equations are recast into ordinary differential equations, such that the fluid properties are described along some characteristic path. Hence, the continuity and momentum equations can be combined and manipulated to give the following result:

$$
\begin{aligned}
& u+\frac{2 C}{\gamma-1}=J^{+}=\text {Const. } \\
& u-\frac{2 C}{\gamma-1}=J^{-}=\text {Const. }
\end{aligned}
$$

These equations are the well known Riemann invariants which are valid along the following paths in the length-time plane.

$$
\begin{array}{ll}
\frac{\partial r}{\partial t}=u+C ; C^{+} \text {Characteristic } & (I I-5 a) \\
\frac{\partial r}{\partial t}=u-C ; C-\text { Characteristic } & (I I-5 b)
\end{array}
$$

The above four expressions are fundamental to the analysis of LSD blast waves near the detonation front.

As previously mentioned, the pressure decay process for the rest of the blast wave is approximated using self-similar cylindrical blast wave theory. Expressions predieting this type of wave decay are easily derived from dimensional analysis. 
Assume that the column of high pressure plasma generated by a LSD wave can be approximated by a cylindrical blast wave resulting from an intense line source explosion. See Figure 16. The dependent variables of this blast wave expansion are velocity $(\mathrm{U})$, pressure $(\mathrm{P})$, and density (P), while the independent variables are blast wave radius $(R)$, and time $(t)$. The physical constants of this decay problem are the energy released during the explosion, as well as, the initial density and pressure. The dimensions of these variables are:

$$
\begin{aligned}
\{E\} & =\frac{K M}{s^{2}} & (I I-6 a) \\
\{r\} & =M & (I I-6 b) \\
\{\rho\} & =\frac{K}{M^{3}} & (I I-6 c) \\
\{t\} & =8 & (I I-6 d)
\end{aligned}
$$

It can be shown, that the initial pressure is not important in strong shock waves; thus, the only parameter constants are the energy released and the initial air density. The objective of dimensional analysis is to form nondimensional parameter groups. Observe that the two independent variables can be combined to form the following similarity parameter:

$$
\eta_{0}=\frac{r}{\left(\frac{E}{p_{0}}\right)^{1 / 4} t^{1 / 2}}=\text { Const. }
$$

Hence, $\mathrm{r}$ is proportional to $t^{-1 / 2}$. Using the strong shock assumption, following expression can be obtained:

$$
P=\left(\frac{2}{\gamma+1}\right) \rho_{0} u^{2}
$$

Differentiating Eqn. 7 and substituting it into the above expression results in an expression for pressure:

$$
P=\frac{1}{2}\left[\frac{\eta_{0}^{2}}{\gamma+1} \rho_{0}\left(\frac{E}{\rho_{0}}\right)^{\frac{1}{2}}\right] t^{-1}
$$

In the above equation the bracketed terms are constant and it is possible to form the following ratio:

$$
\frac{P}{P_{\text {ref }}}=\left(\frac{t}{t_{\text {ref }}}\right)^{-1}
$$

An additional ratio, can be derived, using Eqn. 7:

$$
\frac{r}{r_{r e f}}=\left(\frac{t}{t_{r e f}}\right)^{\frac{1}{2}}
$$

Hence, if blast wave conditions are known for a given time, then the above expressions determine subsequent blast wave pressure decay. 


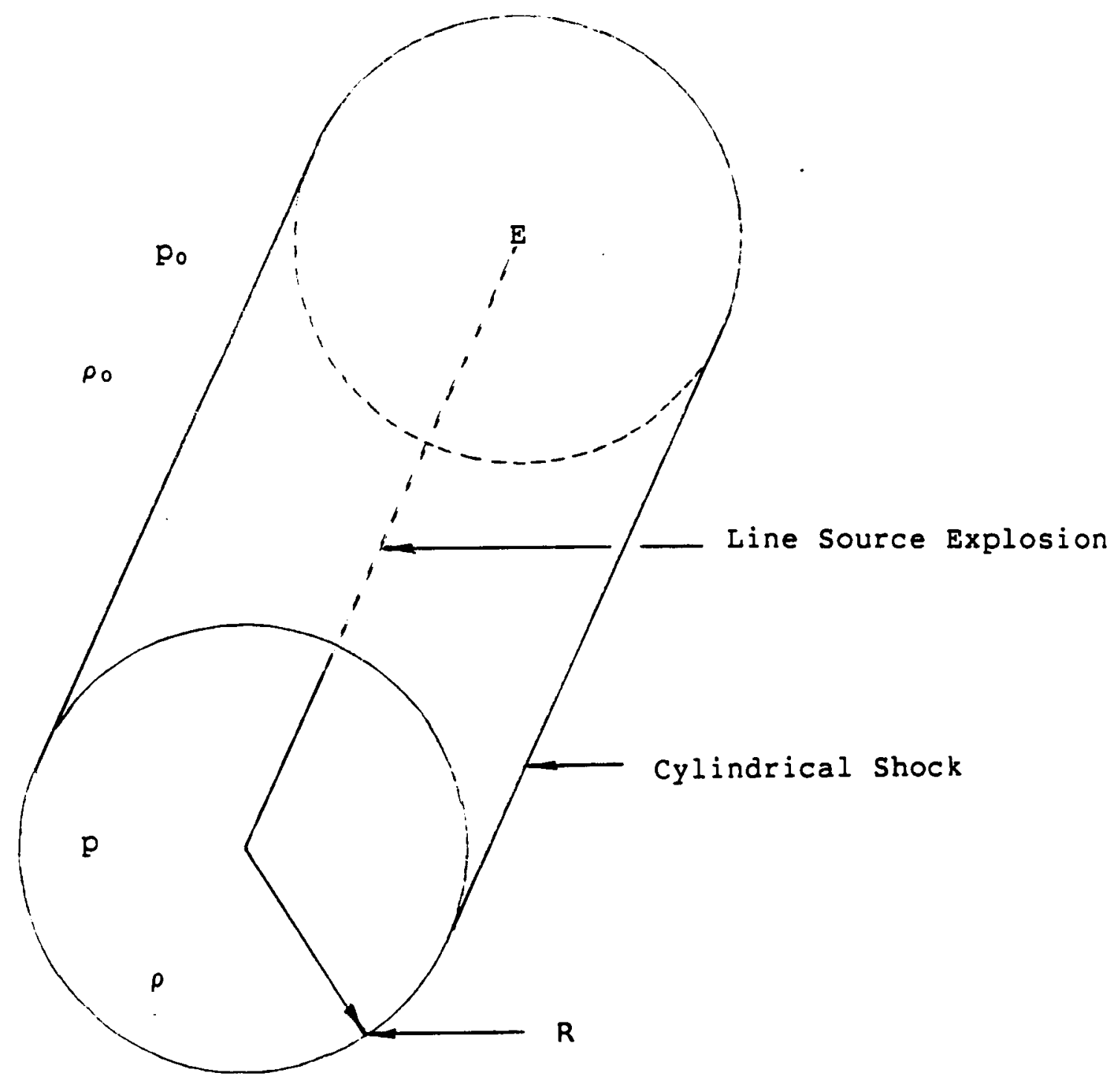

Figure 16. Cylindrical blast wave geometry. 
To determine the fluid conditions directly behind the absorption zone, in the ChapmanJouguet plane, the following equations developed for the ZND model can be used:

$$
\begin{gathered}
u_{C J}=\frac{V_{D}}{\gamma+1} \\
C_{C J}=\left(\frac{\gamma}{\gamma+1}\right) V_{d} \\
C_{C J}=\gamma u_{C J}
\end{gathered}
$$

Recall that the Chapman-Jouguet plane is located just behind the reaction zone; in the case of LSD waves it is behind the absorption zone. Chapman-Jouguet theory predicts that stable detonation waves exist only when the relative velocity of the flow in the Chapman-Jouguet plane, with respect to the wave front, is equal to the local sound speed; therefore:

$$
V_{D}-\varkappa_{C J}=C_{C J}=\left[\frac{\gamma P_{C J}}{C_{C J}}\right]^{\frac{1}{2}}
$$

Combining the definition of the ideal gas sound speed, the expression for polytropic expansion and Eqns. $12 c$ and 13 the pressure behind the detonation is given by:

$$
\left.P_{C J}=\frac{\rho_{0} V Z}{\gamma+1}=\frac{\left[2\left(\gamma^{2}-1\right)\right]^{2}}{\gamma+1} \rho \Phi\right\}
$$

Assuming that the axial velocity is zero, the initial impulse pressure and sound speed are given by the following expressions, respectively:

$$
\begin{gathered}
P_{1}=P_{C J}\left(\frac{\gamma+1}{2 \gamma}\right)^{\left(\frac{2 \gamma}{\gamma-1}\right)} \\
C_{1}=\frac{V_{D}}{2}
\end{gathered}
$$

Using the above results, the Riemann invariants and the self- similar expansion expressions, it is now possible to determine the expansion of the blast wave and subsequent thrust generation. The pressure decay near the detonation front is now examined.

From Fig. 17 it is seen that the first rarefaction fan is a simple backward facing wave; thus, flow conditions behind it can be calculated using the invarient along the $C^{+}$characteristic. Because the radial velocity ahead of the expansion fan is equal to zero, the invariant is written as:

$$
\frac{2}{\gamma-1} C_{1}=u_{2}+\frac{2}{\gamma-1} C_{2}
$$

Combining this with the perfect gas relations, the above equation can be written as:

$$
u_{2}=\frac{2}{\gamma-1}\left(C_{1}-C_{2}\right)
$$



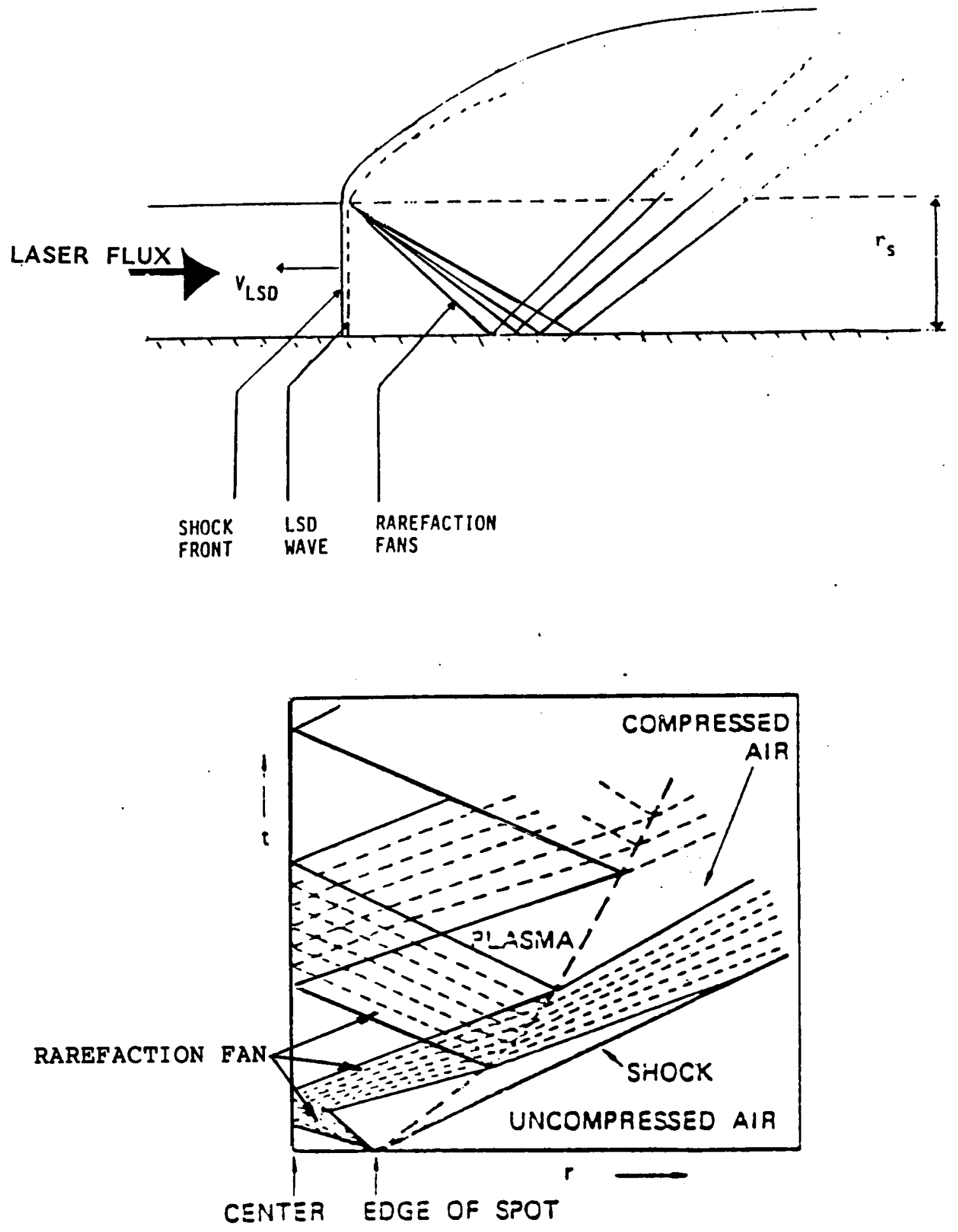

Figure 17. LSD/Blast weve expansion. 


$$
\begin{gathered}
u_{2}=\frac{2}{\gamma-1}\left[\left(\frac{\gamma P_{1}}{\rho_{1}}\right)^{\frac{\gamma}{2}}-\left(\frac{\gamma P_{2}}{\rho_{1}}\right)^{\frac{1}{1}}\left(\frac{P_{1}}{P_{2}}\right)^{\frac{1}{\gamma}}\right] \\
u_{2}=\frac{2}{\gamma-1}\left(\frac{\gamma P_{1}}{\rho_{1}}\right)^{\frac{1}{2}}\left\{1-\left(\frac{P_{1}}{P_{2}}\right)^{\frac{\gamma-1}{\gamma}}\right\}
\end{gathered}
$$

This expression can then be solved numerically with the following well established shock tube result, to determine the pressure behind the rarefaction fan $\left(P_{r 1}\right)$.

$$
\left.\left.u_{2}=\left(\frac{\gamma_{0} P_{0}}{\rho_{0}}\right)^{\frac{1}{2}} \mid \frac{P_{2}}{P_{0}}-1\right] \mid \frac{\frac{2}{\gamma_{0}}}{\left(\gamma_{0}+1\right) \frac{P_{2}}{P_{0}}+\left(\gamma_{0}-1\right)}\right)^{\frac{1}{2}}
$$

Substituting this result into equation $18 \mathrm{c}$, the particle velocity behind the refraction fan can be determeined. Knowing the particle velocity, it is now possible to find the location of the radial shock front. At $X^{\prime}$, the radius of the blast wave is defined as $R_{r e f}$. This reference radius is used to start the self-similar analysis.

\section{STATIC THRUST MODEL}

The impulse loading on the thruster surface due to an expanding cylindrical plasma blast wave, is given by the integral relation:

$$
I=\iint P d A d t
$$

Using the above expression, the impulse delivered to the thruster plate can now be found.

To determine the impulse contribution of Region 1 (see Fig 15) note that its geometry remains fxed as the LSD wave propagates over the thruster surface. The location of $X^{\prime}$ can be determined by integrating Eqn. 5b, solving for the integration constant and multiplying the result by $V_{D}$.

$$
X^{\prime}=2 R_{8}
$$

Therefore the impulse contribution of this region is simply:

$$
I_{1}=2 r_{s}\left(r_{,} P_{1}+r_{\text {ref }} P_{2}\right) t
$$

where the $t$ is equal to the time necessary for the detonation front to propagate across the thruster surface. Since the laser pulse is terminated when the detonation front reaches the end of the thruster plate, $t$ is equal to the laser pulse duration time $t_{p}$. 
The impulse contribution for the region $X>X^{\prime}$ in Fig. 18, can be determined from equations 10, 11 and 20, which are combined to give:

$$
I_{2}=r_{\text {ref }} P_{\text {ref }} \Delta x_{i} \int_{t_{\text {ref }}}^{t_{0}}\left(\frac{t}{t_{\text {rof }}}\right)^{-\frac{1}{2}} d t=2 r_{\text {ref }} P_{\text {ref }} t_{\text {ref }} l\left(\frac{t_{0}}{t_{\text {ref }}}\right)^{\frac{t}{t}}-1 \mid \Delta x_{i}
$$

where $t_{0}$ is the time necessary for the blast wave to decay to the local ambient pressure, and $t_{r e f}$ is the time required for the first rarefaction fan to reach the blast wave center. To account for varying flow properties along the plug nozzle, the impulse in the radial expansionis regime is numerically integrated over the thruster surface, using a given step size of delta $x$. Thus, the total impulse generated by the LSD wave is the sum of the contributions from the two expansion regimes, as given below:

$$
I_{\text {TOTAL }}=I_{1}+\sum_{i=1}^{N} I_{i}
$$

Note that the impulse force acts along the thruster's surface normal. For the ERH thruster engine configuration examined, all of the impulse loading predicted by the above expression does not contribute to engine thrust; only the impulse vector component aligned with the vehicle flight direction. Thus, magnitude of the impulse component resulting in upward thrust is:

$$
I_{\text {USABLE }}=I_{\text {TOTAL }} \cos \alpha
$$

where $\alpha$ is the base angle of the truncated conical plug.

In trajectory calculations is convenient to use time averaged thrust. This mean thrust is defined as:

$$
T=\frac{1}{t_{\text {CYCLE }}} \int_{t}^{t_{t} \text { toYaLE }} I d t=\frac{I_{\text {TOTAL }}}{t_{\text {CYCLE }}}
$$

where $I$ is the impulse delivered per laser pulse, and $T_{\text {cycle }}$ is the blast wave period. The blast wave period is simply the total expansion time of the LSD blast wave, plus the refresh time; as given by:

$$
t_{\text {CYCLE }}=\frac{1}{P R F}=t_{0}+t_{\text {RERRESH }}
$$

The refresh time is the time necessary for the hot expanded gas to clear the thruster surface and be replaced by the ambient air. Currently, an adequate model for the refresh process does not exist and in this analysis the refresh time is set equal to zero.

Input energy per laser pulse is evaluated as follows:

$$
E_{P}=\frac{\pi R_{0}^{2}}{2} \Phi t_{p}
$$

In the derivation of this equation it is assumed that the laser beam has a semicircular shape and uniform intensity. Recall that the laser energy is absorbed in a thin region behind the detonation 
front. Thus, $t_{p}$ is the time requried for this LSD wave to transverse the ERH thruster plate. To convert the pulse energy to time average laser power the following equation is used:

$$
P_{\text {AVE }}=\frac{E_{P}}{t_{\text {CYCLE }}}
$$

Finally, an important measure of thruster efficiency is the coupling coefficient, which is the ratio of usable single- pulse impulse to laser input energy per pulse. Therefore, the coupling coefficient is defined by:

$$
C C=\frac{I_{\mathrm{USABLE}}}{E_{P}}=\frac{T}{P_{\mathrm{AVE}}}
$$

\section{DYNAMIC THRUSTER MODEL}

The above analytical model does not consider the effects of flight platform velocity on ERH thruster performance. This model is sufficient for static and low subsonic flight velocities, but when flight velocities are supersonic, ram drag, compressiblity effects and flow expansion over the aft plug nozzle (the thruster surface) become important. For an airbreathing engine net thrust is the force resulting from the addition of all pressure and viscous forces, excluding the external drag forces; that is, gross thrust minus ram drag. Note external drag forces caused by the "installed" engine are typically included with vehicle drag. As a result, it was necessary to develop a dynamic model for ERH thruster performance.

In supersonic flight, an attached oblique shock forms at the conical spike tip. The air is compressed after passing through this oblique shock, and it is turned to flow tangent to the forebody surface; the entire forebody of the flight platform is essentially an isentropic spike inlet. The annular cowl, which circumscribes the craft's midsection, then redirects the flow parallel to the thruster surface. In the ERH thruster operation, the cowl only turns the flow, and does not decelerate or accelerate it. Additionally, the cowl is assumed to have a sufficiently large capture area such that no spillage occurs beyond the design mach number of three. With these cowl characteristics, no normal shock forms at the cowl entrance and the exit mach number is equal to that at the cowl entrance. Upon leaving the cowl, the ducted air expands along the thruster surface as if it where emerging from a free expansion type plug nozzle.

Therefore the only stagnation significant pressure loss occurs as the air passes through the oblique shock. To calculate such losses, an experimentally determined pressure recovery inlet schedule developed by Marquart[15| for the XRJ59-MA-3 inlet was used. In the present analysis, it is also assumed that the air flow through the cowl remains undisturbed by the LSD wave propagation and subsequent blast wave expansion. Such an assumption appears to be justified, when the maximum expansion of the blast wave is small compared to cross sectional flow area of the cowl. 
In supersonic fight, pressure variations along the thruster surfece become pronounced and must be considered when calculating the LSD blast wave expansion. When calculating this pressure variation, it is assumed that the compressed air flow leaving the cowl expands as shown in Figure 19. To calculate the pressure for a given thruster surface location (s) it is necessary to determine the local cross sectional area of the expanding gases.

Typically, for a non-truncated plug nozzle, the pressure at the end of the plug is always equal to $P_{0}$, the ambient pressure. Using this condition and assuming isentropic flow over the plug the following expression can be solved for the mach number at the plug end $\left(M_{e}\right)$.

$$
\frac{P_{T c e}}{P_{0}}=\left(1+\frac{\gamma-1}{2} M_{e}^{2}\right)^{\frac{\gamma}{\gamma-1}}
$$

where $P_{t c e}$ denotes the stagnation pressure at the cowl exit. As previously mentioned this is determine using the Marquardt inlet data. Once $M_{\varepsilon}$ is known, the cross-sectional flow area, $A_{e}$, can be obtained using:

$$
\frac{A_{c e}}{A_{e}}=\frac{M_{e}}{M_{c e}}\left[\frac{2+(\gamma-1) M_{c e}^{2}}{2+(\gamma-1) M_{e}^{2}}\right]^{\frac{\gamma+1}{\gamma-1}}
$$

where $A_{c e}$ and $M_{c e}$ are the cross-sectional flow area and the mach number at the cowl exit respectively.

From the nozzle's geometry:

$$
R_{e}=\sqrt{\frac{A_{e}}{x}}
$$

If the cross-sectional flow area is assumed to vary linearly along the thruster surface, then:

$$
A=\pi\left[R_{e}^{2}-\left(\frac{8}{\cos \alpha}\right)\right]
$$

In this equation, $\alpha$ is the plug base angle and $s$ denotes an arbitrary position along the plug surface. For a given s, the coresponding mach number can be found implicitly using:

$$
M(s)=\frac{A_{\theta} M_{e}}{A(s)}\left|\frac{2+\frac{x-1}{2} M^{2}}{1+\frac{x^{-1}}{2} M_{e}^{2}}\right|^{\frac{x+1}{2-1}}
$$

Now the local expansion pressure can be calculated from:

$$
P(s)=\frac{P_{\text {Tce }}}{\left(1+\frac{2-1}{2} M(s)^{2}\right)^{\frac{\gamma}{7-1}}}
$$

In the dynamic model, the LSD blast waves must expand to this pressure.

To calculate the net thrust, a control volume is constructed as shown in Fig. 20. By choosing the control volume boundary adjacent to ERH thruster surface, the complex flow conditions due to the interaction between the blast wave expansion and the air flow directed by the cowl can be neglected; this simplifies the drag analysis. 

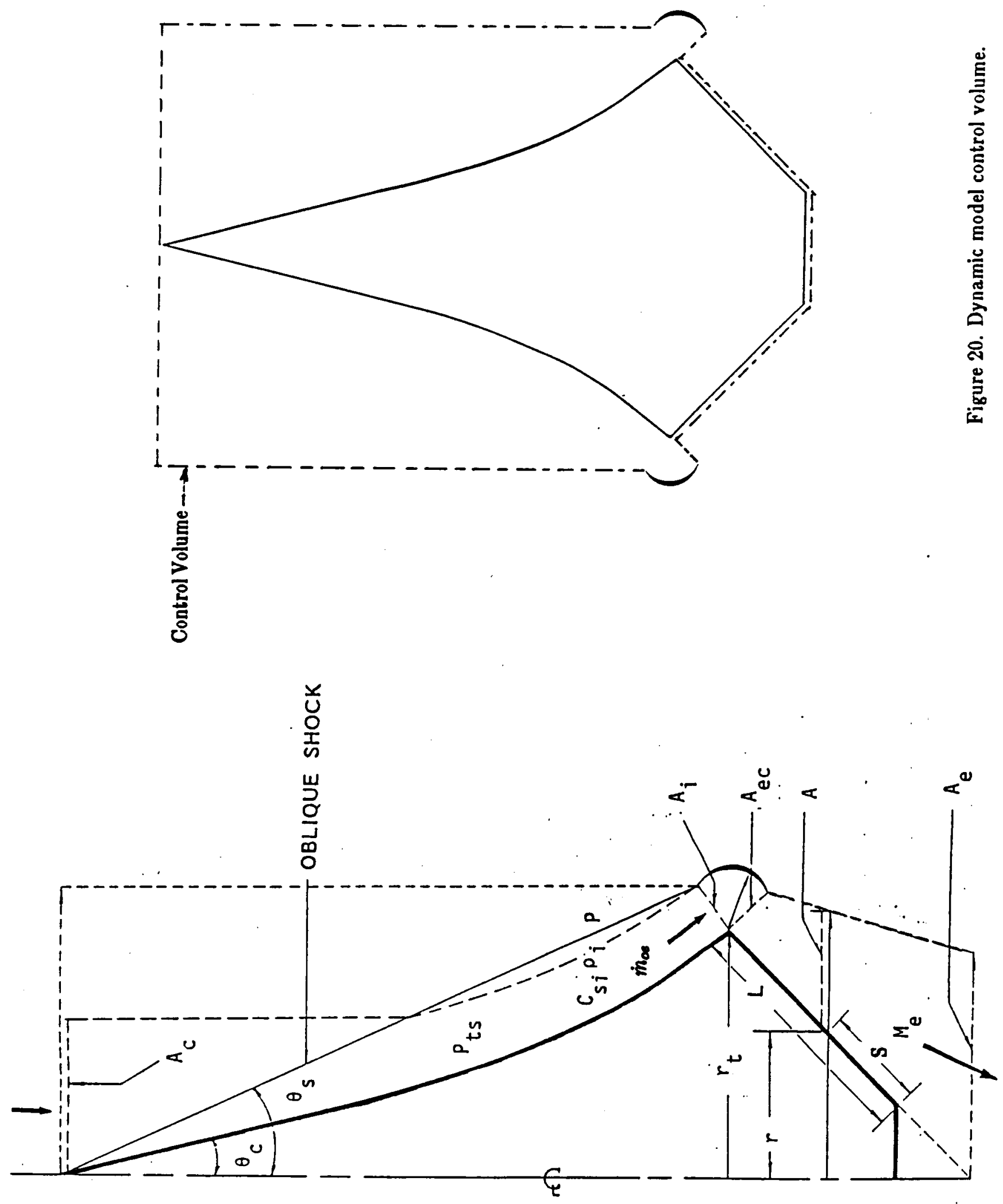

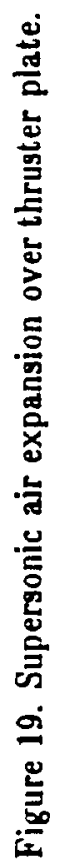


Recall that in the development of the LSD blast wave model, the ambient pressure was already subtracted from the integrated impulse, hence it is necessary only to consider the pressure difference across the cowl. The verticle force due to this pressure difference is given by:

$$
\text { Pressure Drag }=\left(P_{0}-P_{c e}\right) A_{c e} \sin \alpha
$$

Note that there is a force due to the momentum change of the incoming air flow is redirected to convect over the thruster surface. This force is calculated using the following expression:

$$
\text { Cowl Drag }=\dot{m}_{c e} V_{c e} \sin \alpha
$$

Therefore the net thrust is given by:

$$
T_{\text {net }}=T-\text { Pressure Drag }- \text { Cowl Drag }
$$

\section{E. RDSULTS}

Performance maps for the ERH thruster engine were generated by incorporating the preceding static and dynamic models into a comprehensive computer simulation. Using this computer model, the effects of flight mach number and altitude on net engine thrust, as well as several other performance parameters was investigated.

For the Apollo Lightcraft, the following ERH engine configuration was assumed. As previously mentioned, the thruster surface is a truncated conical plug which also comprises the entire aft section of the vehicle. This plug has a semi-vertex angle of 45 degrees. During ERH thruster operation, 48 LSD waves are ignited; they are equally spaced around the circumference of the plug. The laser beams which initiate these waves are assumed to provide a uniform intensity of $5 x 10^{8} \mathrm{~W} / \mathrm{cm}^{2}$, over the semi-circular spot of $0.5 \mathrm{~cm}$ radius.

In Fig. 21 net thrust variation is presented as a function of flight mach number and altitude. Recall that net thrust is the time-averaged thrust minus ram drag. For a given altitude, observe that thrust drops off at some critical mach number. This is due, in part, to the increased ram drag experienced by the ERH thruster engine at higher mach numbers.

Fig. 21 also shows that mean thrust decreases with increasing altitude. This result is not unexpected since at higher altitudes atmospheric pressure and density decrease. The ERH thruster, like other airbreathing engines, relies on momentum transfer of incoming ambient air to generate thrust; therefore, as the density decreases, so does engine thrust. 


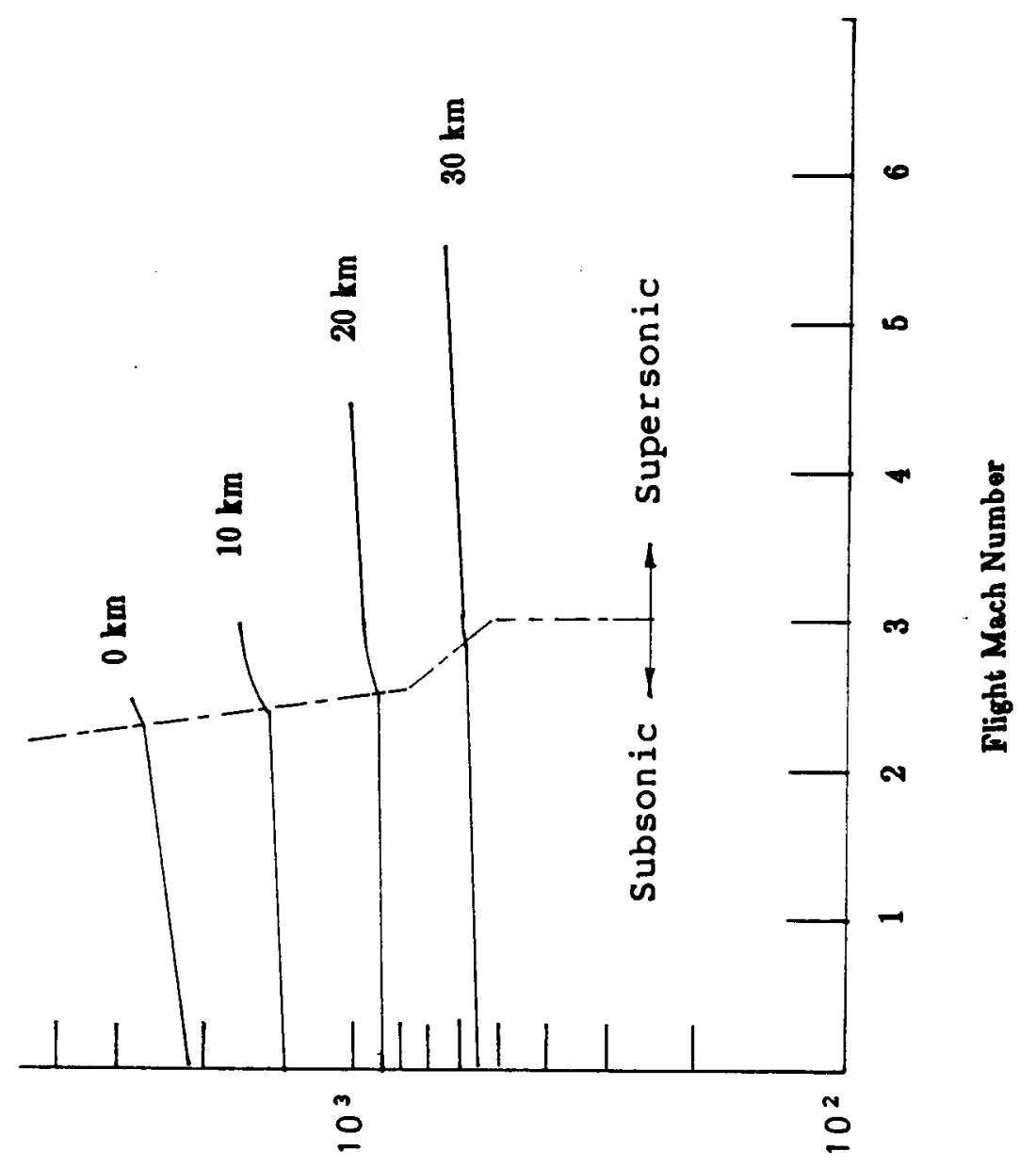



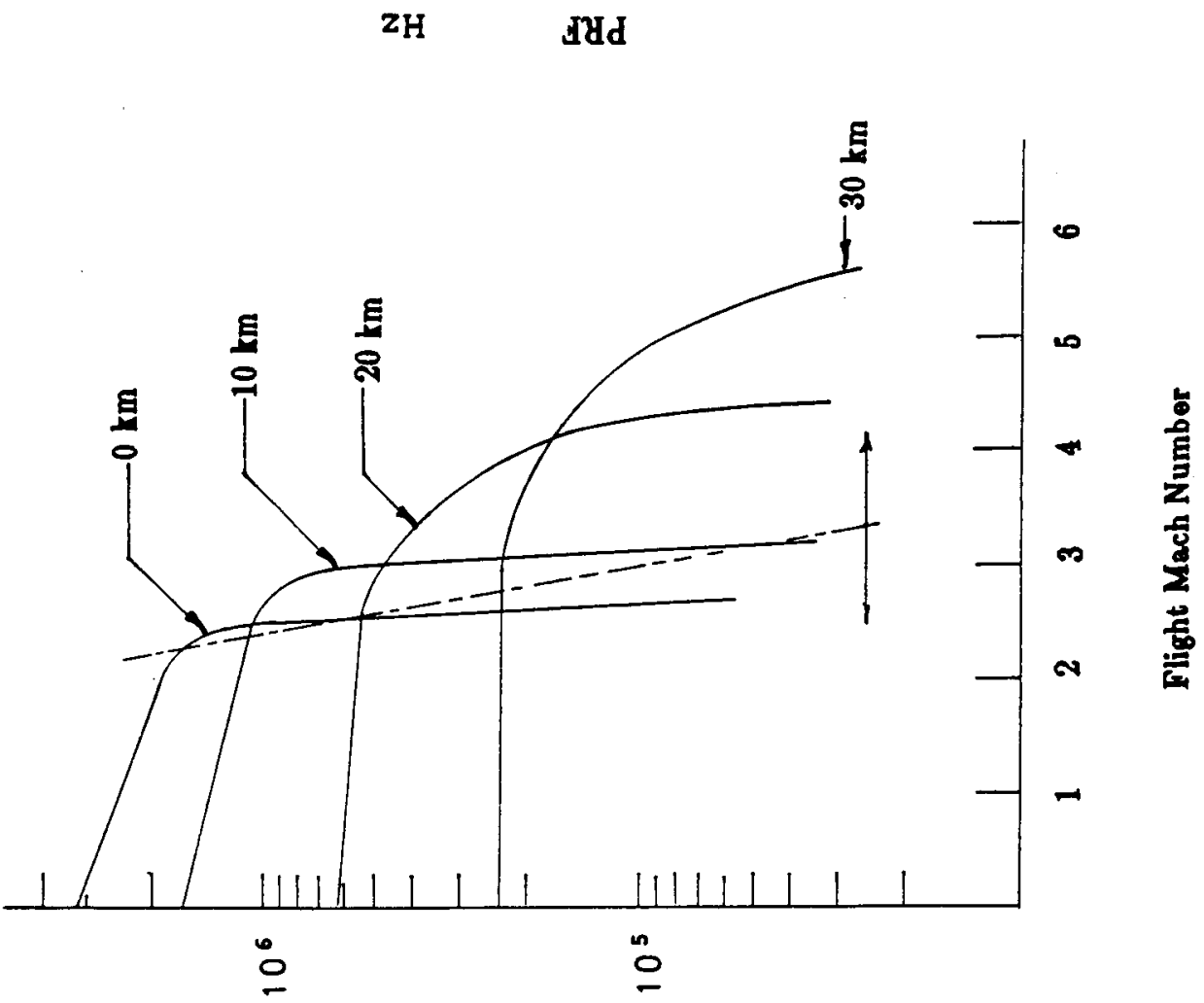

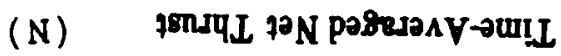


Another factor which causes a reduction in net thrust, for this ERH engine configuration, is illustrated in Fig 22: the maximum pulse repetition frequency (PRF) rate. Note that PRF decreases with increasing altitude. During the ERH thruster operation, LSD blast wave pressure must expand to local ambient pressure before the thruster surface can be refreshed and new LSD waves initiated. At higher altitudes, the local static pressure is lower, 80 the blast wave expansion time $\left(t_{0}\right)$ is longer. Since the high-internal pressure of the plasma blast wave decreases rapidly as the wave expands, most of the engine thrust is generated during the initial blast wave decay. Therefore, an increase in the LSD blast wave expansion time $\left(t_{0}\right)$ does not greatly increase the impulse delivery to the thruster plate, and time averaged thrust must decrease.

Closely related to the PRF is the time-averaged laser power; its variation with altitude and flight mach number can be seen in Fig. 23. Also, in Fig. 24, the predicted coupling coefficient performance is plotted, with the dotted line indicating the theoretical limit for coupling coefficient of airbreathing engines. Since the calculated coefficients at high altitudes (e.g., $30 \mathrm{~km}$ ) so closely approach the theoretical limit, it is suspected that this first order analysis of ERH engine performance may be overly optimistic. There are a number of reasons for these high values.

In the current model, as mentioned above, the refresh time ( $\left.t_{\text {refresh }}\right)$ is set to zero. In an actual engine, $t_{\text {rofreen }}$ would have some finite value, which would cause a decrease in the PRF and hence, the time averaged thrust. A second model simplification, which probably contributes to producing optimistic results, is the omission of axial rarefaction waves within the LSD blast wave simulation. Inclusion of axial expansion effects in this blast wave model, would result in a reduction in the impulse delivery to the thruster surface. At the present time, the combined effects of these simpliflcations on ERH thruster performance have yet to be quantifled. Therefore, to be conservative the net thrust values used in the trajectory analysis are reduced by $33 \%$. 

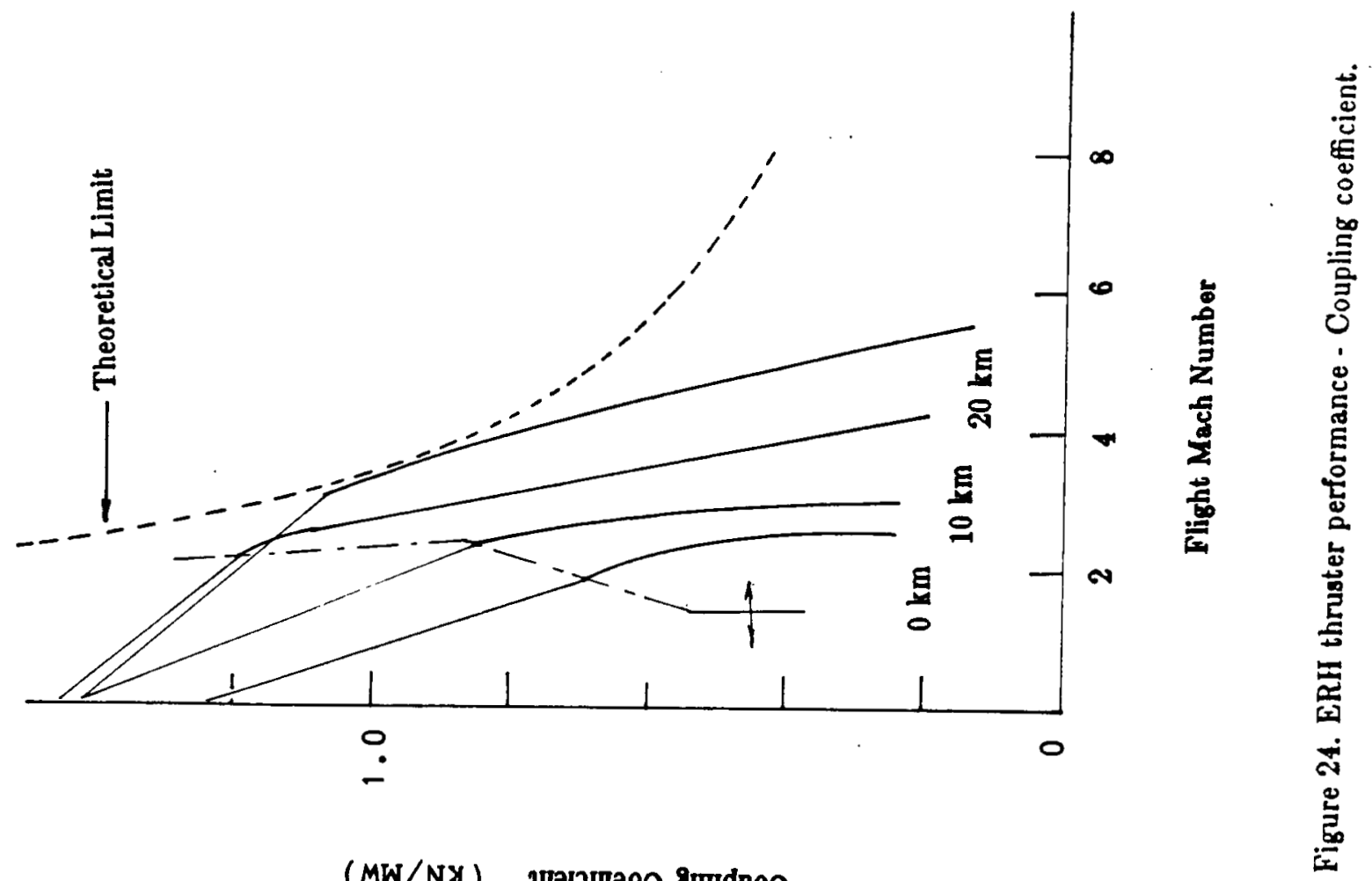

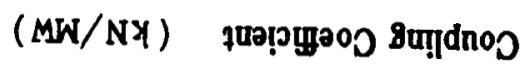

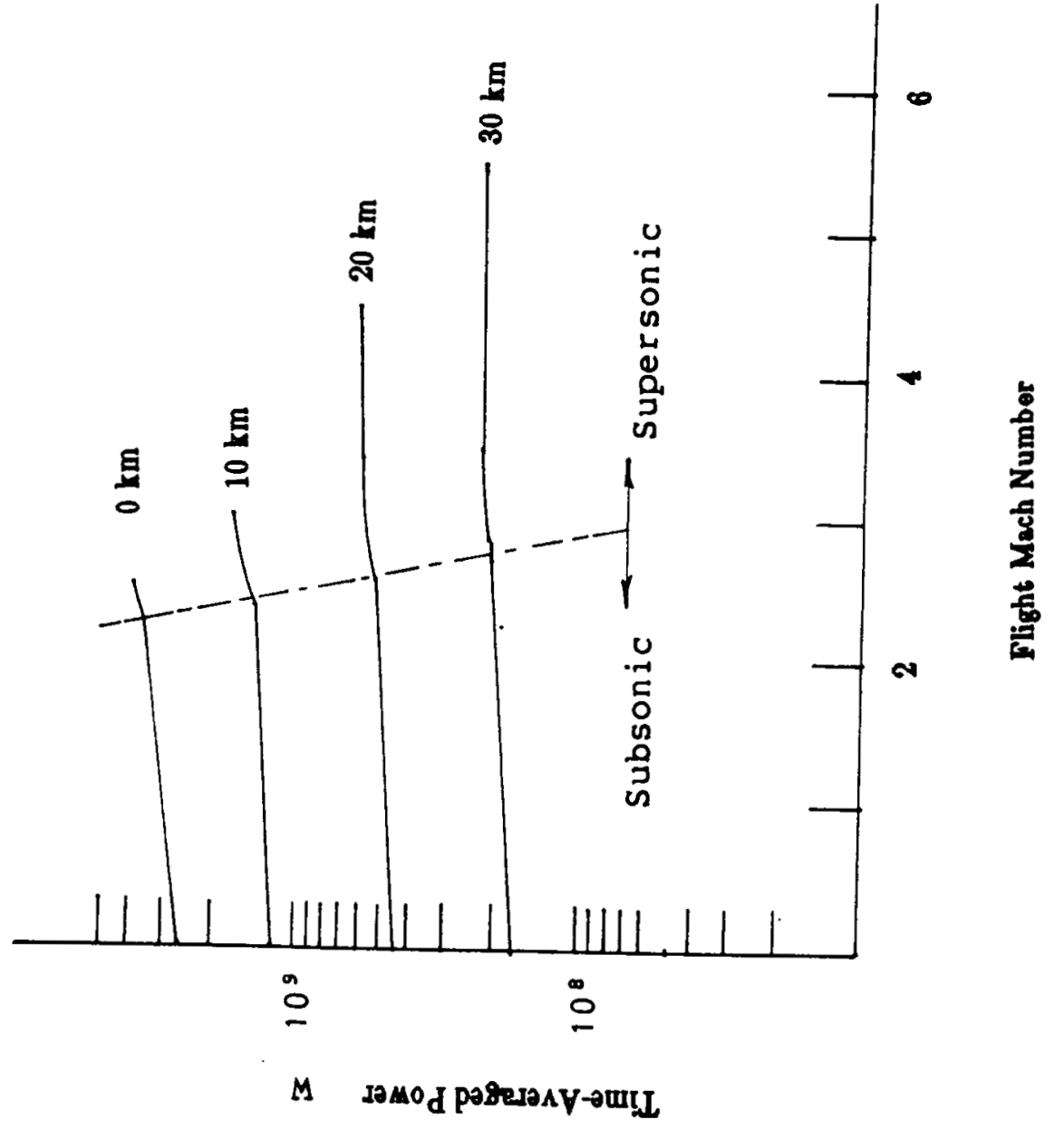

 


\section{CHAPTRR II}

\section{RAMJET/SCRAMJET MODE}

The scramjet engine will begin operation at about Mach 3.0 and 20000ft, where the ERH thruster cuts off. The scramjet engine produces thrust by adding heat to supersonic internal engine air. In current scramjets, liquid chemical fuel is burned to heat the engine working fluid. In the Lightcraft engine, however, energy is added by focusing a laser beam into the combustion chamber (see Fig. 25). The flow is heated to high temperatures by passing through a "planar heater" produced by a repetitively-pulsed LSD wave which propagates radially outward from the centerbody to the cowl. The laser is shut off when the LSD wave reaches the cowl inner lip, where the secondary optics are located.

\section{A. INLET SPIKE}

Since the scramjet is used in supersonic/hypersonic fight from about Mach 3.0 to 12.0, a bow shock will form over the forebody of the vehicle. The incoming flow first encounters a 30 (i.e., half apex angle) degree cone. To determine the external compression inlet performance, it is assumed that the surface flow conditions aft of the bow shock are uniform and equal to those entering the annular cowl duct. This permits the calculation of static pressure, total pressure and surface Mach number along the 30 degree cone (after the initial conical bow shock) as a function of flight Mach number. Additional flow compression caused by the parabolic primary optics surface is assumed to occur through a series of very weak shocks; therefore, only a small loss of total pressure occurs. The bow shock is always assumed to be attached to the cowl with (i.e. no spillage drag). The design Mach number for the inlet is 3.0, and thereafter, the shroud translates aft such that the bow shock is always attached to the shroud forward lip. The mass flow rate entering the vehicle is given by Eqn. (1).

$$
\begin{gathered}
\dot{m}=\rho_{\infty} U_{\infty} A_{c} \\
\rho_{\infty}=\text { ambient air density } \\
U_{\infty}=\text { speed of vehicle } \\
A_{c}=\text { capture inlet area }
\end{gathered}
$$




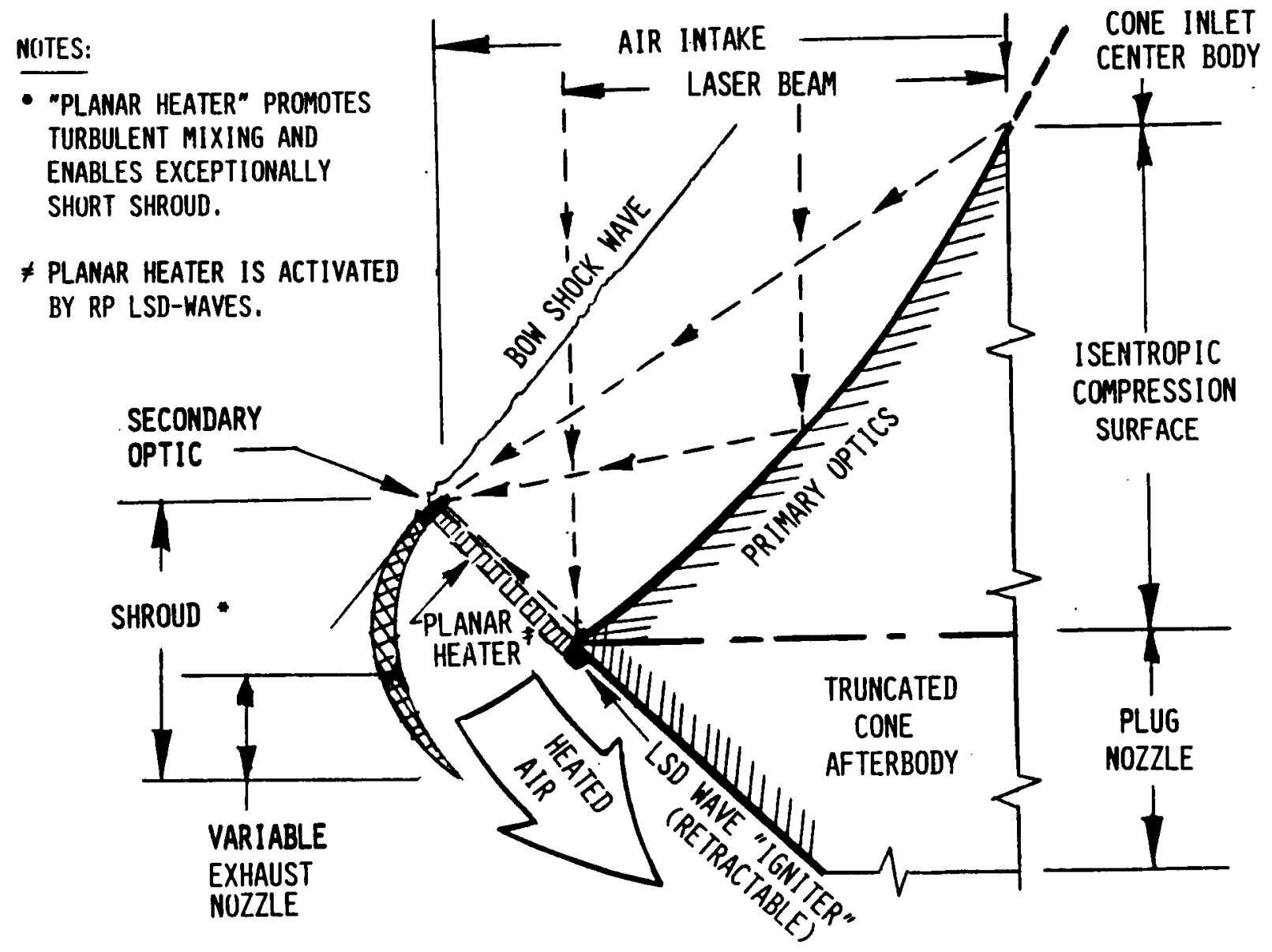

Figure 25. Scramjet configuration. 


\section{B. INTERNAL DIPPUBRR}

In order to reduce internal cow volume, no diffuser was invoked aft of the cowl entrance station. As mentioned earlier, the flow conditions entering the cowl are considered to be the same as those after the bow shock. No normal shock is assumed to form at the cowl lip, as would be typical for ramjet operation.

\section{COMBUSTION CHAMBJR}

Laser energy is absorbed into the flow within the combustion section, which is assumed to have a constant cross-sectional area. The calculations assume $85 \%$ of the incoming beam power appears as an increase in total temperature. The analysis computes the effect of heat addition with Rayleigh line equations for a constant area flow. The ratio of specific heats is set to 1.3 due to the elevated gas temperatures.

Theoretical thermally-choked conditions are computed from the cowl entry Mach number. Laser energy then raises the total temperature of flow according to Eqn.(2).

$$
\begin{gathered}
\Delta T_{0}=\eta_{c} \frac{1}{\dot{m} C_{P}} \dot{Q} \\
\eta_{c}=\text { combustion efficiency }(.85) \\
\dot{m}=\text { air mass flow rate through engine } \\
\dot{Q}=\text { laser power delivered to engine } \\
C_{P}=\text { Specific heat at constant pressure }
\end{gathered}
$$

Heat addition is limited such that the thermally- choked condition cannot be violated. Also the static temperature is not allowed to exceed $10,000 \mathrm{R}$, beyond which the ideal gas assumption is no longer valid. Above 10,000 R, a large fraction of the input laser energy would go into dissociation and ionization losses, which is undesirable. Input beam power is decreased accordingly to hold the temperature below these limits.

\section{PLUG NOZzLE}

In the ramjet mode, flow leaving the combustion chamber is not thermally choked; thus, a slight contraction in the annular duct area is needed to accelerate the flow up to Mach 1.0, prior to expanding over the external plug nozzle. Such nozzle area changes are generally not required for 
scramjet operations, since the flow always leaves the combustion chamber at Mach numbers greater than (or equal to) one. Losses in total pressure due to this contraction are assumed negligible in comparison to other losses.

The flow leaves the shroud and then expands across the bottom surface of the Lightcraft. The 45 degree apex half angle nozzle produces a final exhaust velocity determined by Eqn. (3).

$$
\begin{aligned}
V_{o} & =\left\{2 C_{P} T_{o c} \eta_{m}\left[1-\left(\frac{P_{\infty}}{P_{o c}}\right)^{\frac{\gamma-1}{\gamma}}\right]\right\}^{\frac{1}{2}} \\
V_{o} & =\text { exit exhaust velocity } \\
T_{o c} & =\text { total temperature after heat addition } \\
\eta_{\emptyset} & =\text { nozzle adiabatic efficiency }(.95) \\
P_{\infty} & =\text { ambient air pressure } \\
P_{o c} & =\text { total pressure after combustion } \\
\gamma & =\text { specific heat ratio }(1.3)
\end{aligned}
$$

The net thrust produced by the engine, neglecting the external drag on the shroud and pressure terms, is given by

$$
\begin{gathered}
T_{\text {net }}=\dot{m} V_{0}-\dot{m} U_{\infty} \\
T_{\text {net }}=\text { net thrust } \\
\dot{m}=\text { mass flow rate (air) }
\end{gathered}
$$

where the first term is referred to as gross thrust, and second is the ram drag. Vehicle drag force is computed using the vehicle drag coefficient, which is shown as a function of flight Mach number in Fig. 26.

\section{RAMJET}

A performance analysis for ramjet operation was performed for low supersonic speeds ranging from Mach 2 to Mach 6. A normal shock was assumed to be attached to the cowl lip at all times. Conditions across the normal shock were computed, and Rayleigh line equations to compute the effects of heat addition were used (as with the scramjet).

The results indicate that the ramjet does not give positive thrust until it reaches altitudes and speeds where the scramjet already provides better performance. It was thus decided to abandon 


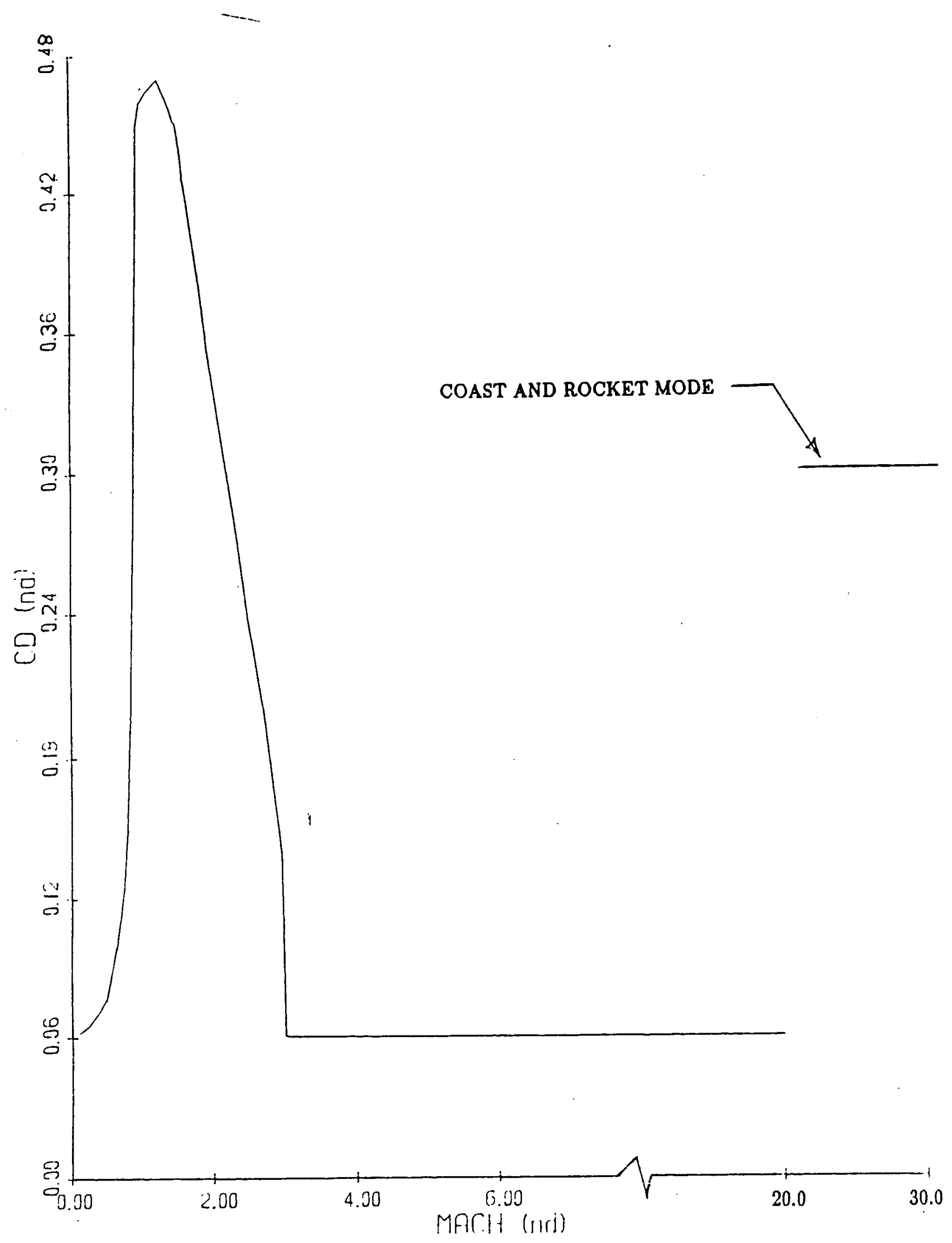

Figure 26. Vehicle drag coefficient. 
the ramjet mode since the scramjet performs better throughout the entire Mach 3-11 regime. The major reason for poor ramjet performance is intimately tied to the cowl design; no internal diffuser was included and large losses in total pressure were caused by the normal shock.

\section{F. RISULTS}

A computer code was assembled to facilitate the analysis of scramjet performance throughout the flight envelope. The program computed thrust, coupling coefficient, propulsive efficiency, laser power and overall efficiency as a function of flight Mach number, with altitude as the parametric variable. Graphs of these results are presented in Figs. 27 through 31. 
Thrust vs. Mach No. (4Gw)

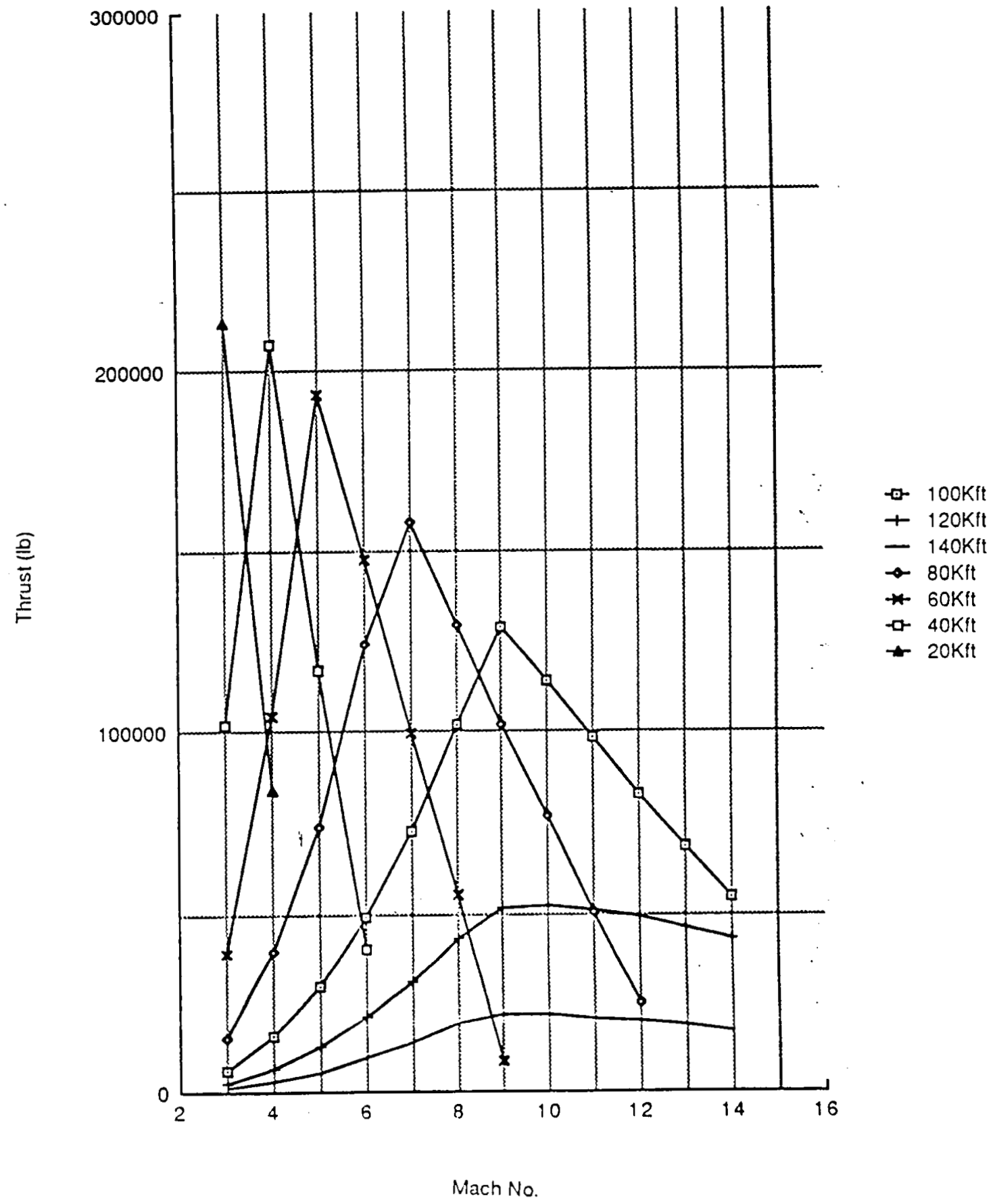

Figure 27. Thrust vs. Mach number for the scramjet. 
Coupling Coefficient vs. Mach No. (4Gw)

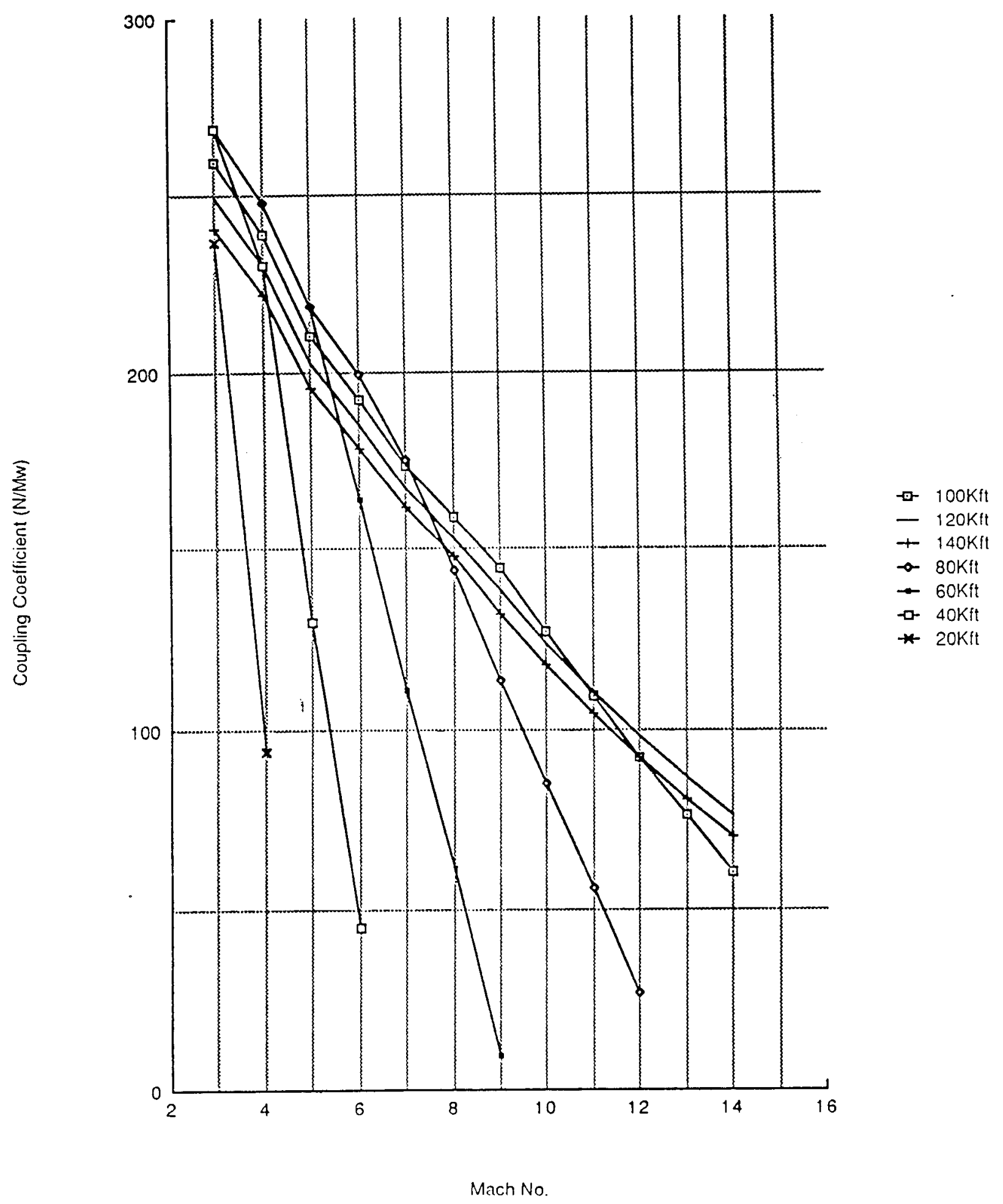

Figure 28. Coupling coefficient vs. Mach number for the scramjet. 
Power vs. Mach No. (4Gw)

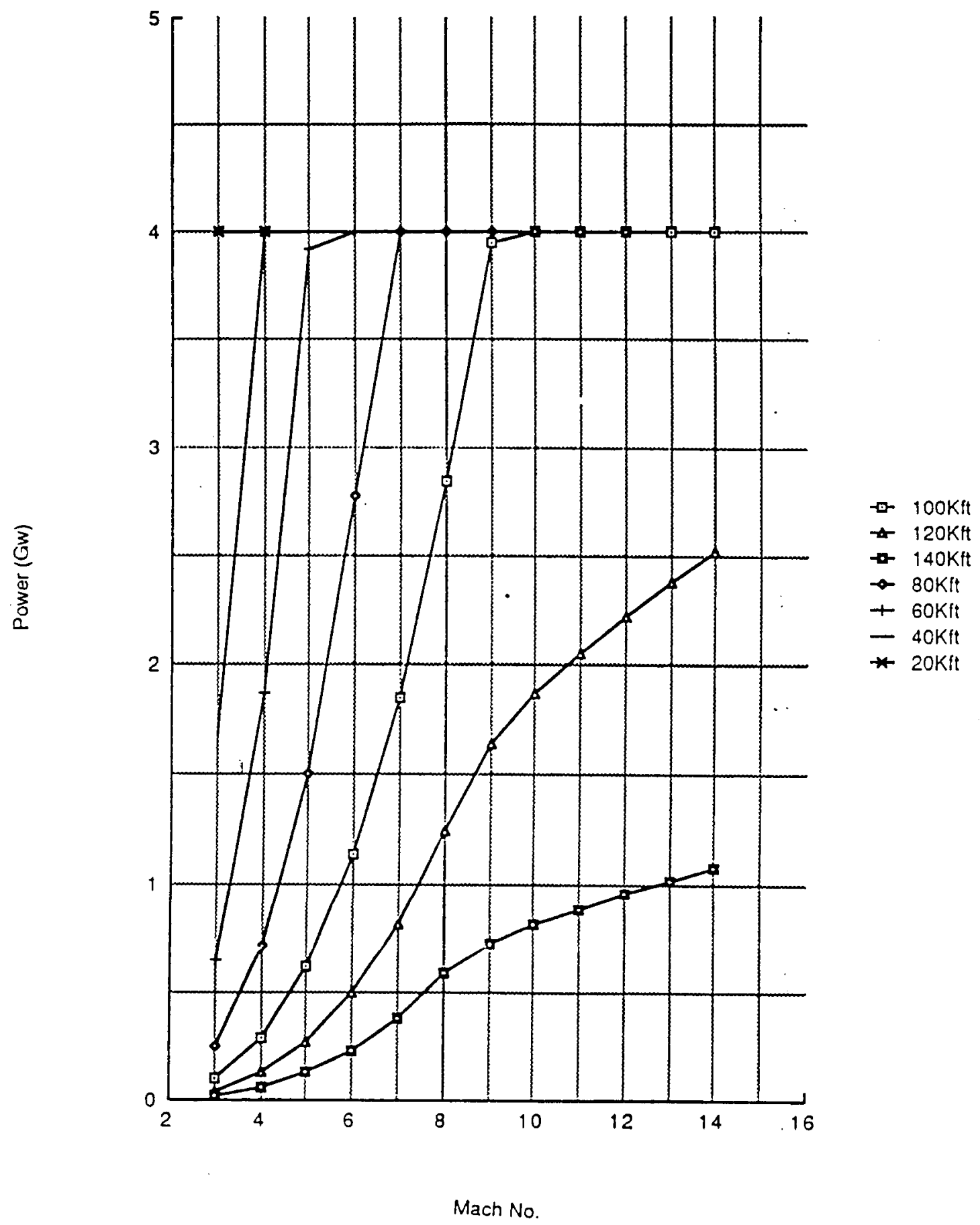

Figure 29. Power vs. mach number for the scramjet. 
Propulsive Efficiency vs. Mach No. (4Gw)

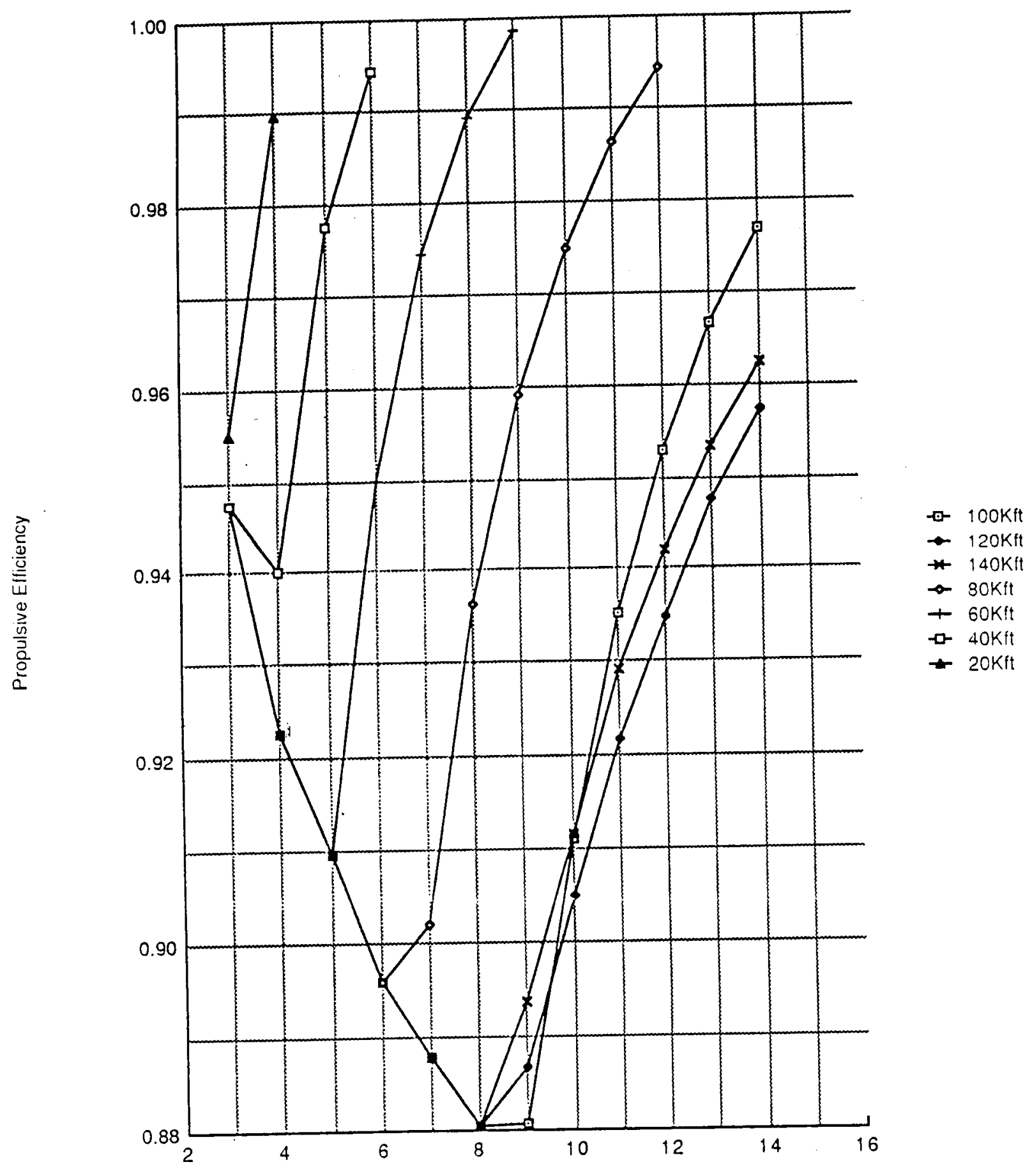

Mach No.

Figure 30. Propulgive efficiency vs. Mach number for the scramjet. 
Overall Efficiency vs. Mach No. (4Gw)

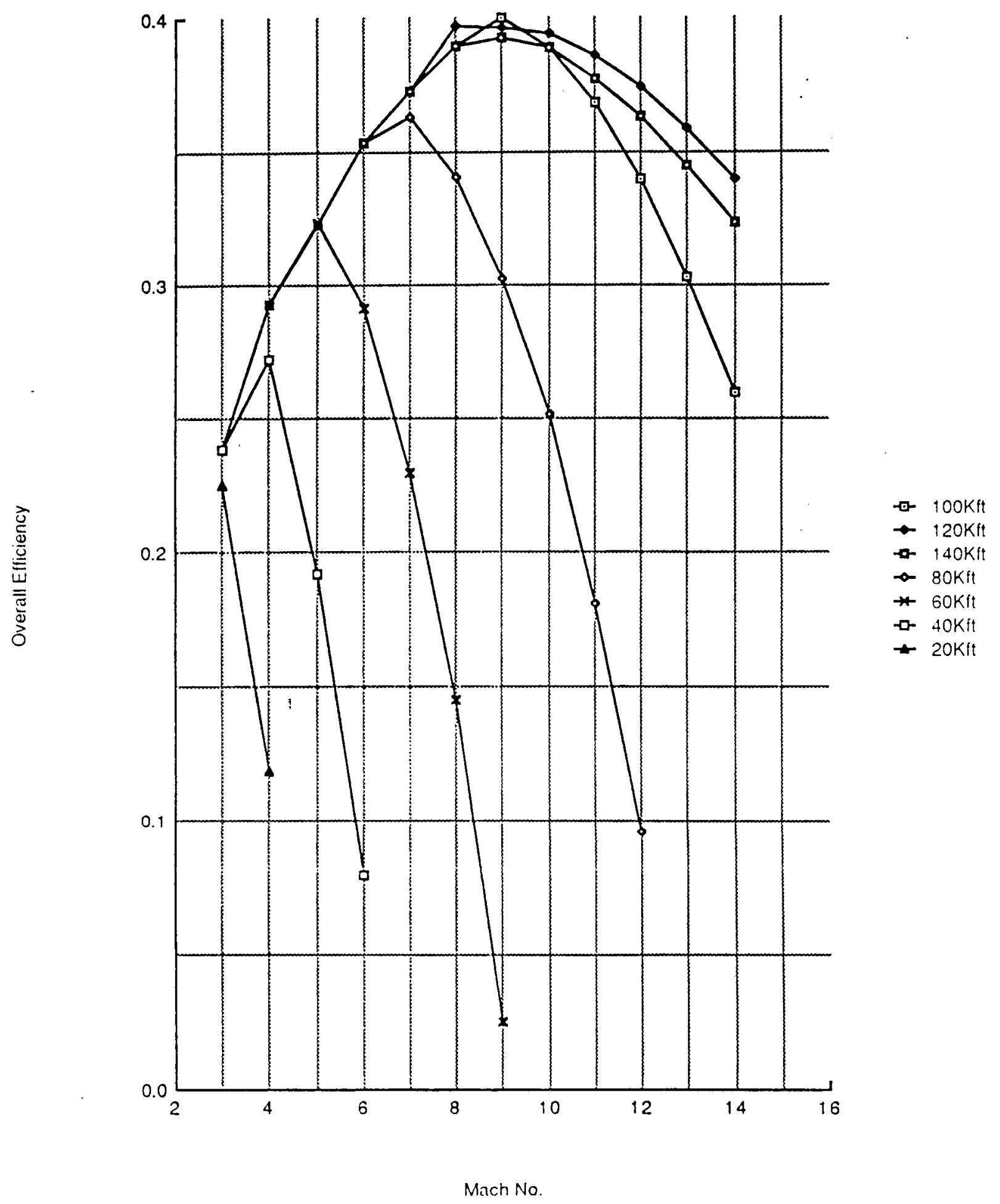

Figure 31. Overall efficiency vs. Mach number for the scramjet. 


\section{CHAPTER IV \\ MHD-FANJET MODE}

The MHD-fanjet is an electric air-turborocket which uses electromagnetic fleld propulsion to accelerate the partially ionized air heated by the bow shock formed over the forebody of the vehicle. The basic cycle is illustrated in Fig. 32. The laser absorption chamber procesess high pressure (30 to $80 \mathrm{~atm}$.) liquid hydrogen propellant into a high temperature $(15,000$ to $20,000 \mathrm{~K})$ ionized gas using a standing laser-supported combustion (LSC) wave. This high temperature, high pressure plasma is then driven via a pressure gradient through an MHD generator, which extracts electrical power from the plasma by applying a magnetic field perpendicular to the core flow (the velocity outside of the boundary layers in the generator duct) and placing electrodes along opposite walls of the generator duct through which the induced current can flow. The load for each pair of generator electrodes is a connection across the MHD accelerator duct. This current flow across the accelerator duct creates the applied electric field, $E_{\text {eppl }}$, for the MHD accelerator. A magnetic field is applied perpendicular to $E_{\text {appl }}$, creating a Lorentz force which accelerates the ionized air flowing through the accelerator. This provides the majority of the thrust during the MHD fanjet mode, the additional thrust resulting from the high velocity hydrogen exhaust of the MHD generators.

\section{A. LASER ABSORPTION CHAMBSR}

A continuous (CW) laser beam from the remote power source is focused into the absorption chamber to a rectangular cross-sectional area small enough (or an intensity large enough) to achieve electrical breakdown in the hydrogen propellant flling the chamber. The laser wavelength is chosen such that the propellant velocity equals the propagation velocity of the LSC wave (toward the laser source), producing a standing LSC wave at the inlet of the MHD generator. The absorption chamber/generator concept is depicted in Fig. 33. For the high aspect ratio at the inlet of the generator, the LSC wave will practically be a line. If this proves to be an unstable geometry, one possible alternative is to use a linear series of smaller circular cross-section LSC waves across the inlet of the generator.

For laser powers larger than $10 \mathrm{~kW}$ and static pressures larger than $3 \mathrm{~atm}$, the hydrogen LSC wave will absorb nearly all of the laser power (over $98 \%$ ). However, radiation heat loss from the LSC wave can seriously reduce the net power going into the gas. In a numerical study by Jeng and Keefer [8] of an axially-symmetric hydrogen LSC wave, a wave velocity of a $100 \mathrm{~m} / \mathrm{s}$, a static pressure of $3 \mathrm{~atm}$, and a laser power of 10 to $60 \mathrm{~kW}$ produced a conversion efficiency of laser power 


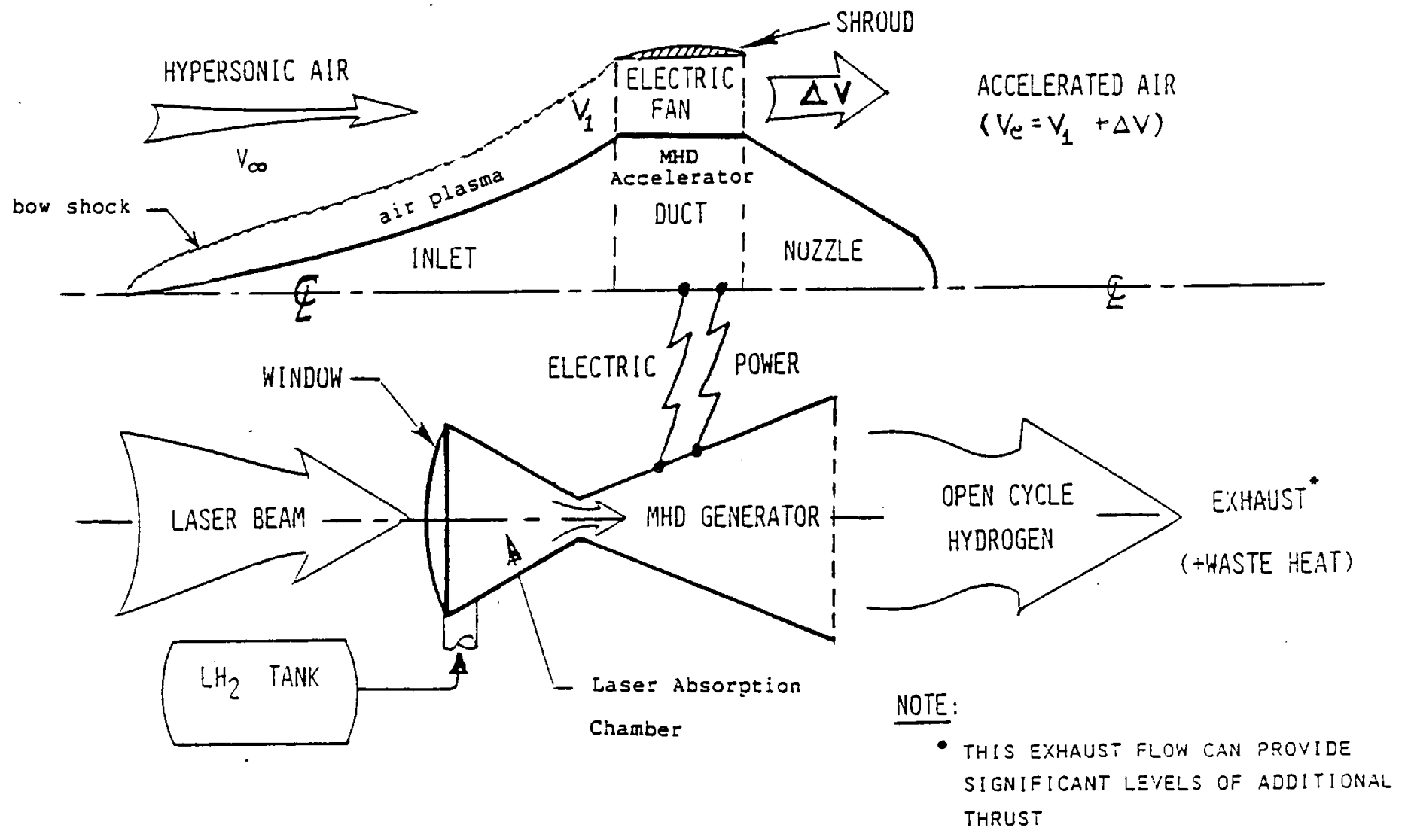

Figure 32. Diagram of the MHD-fanjet cycle.

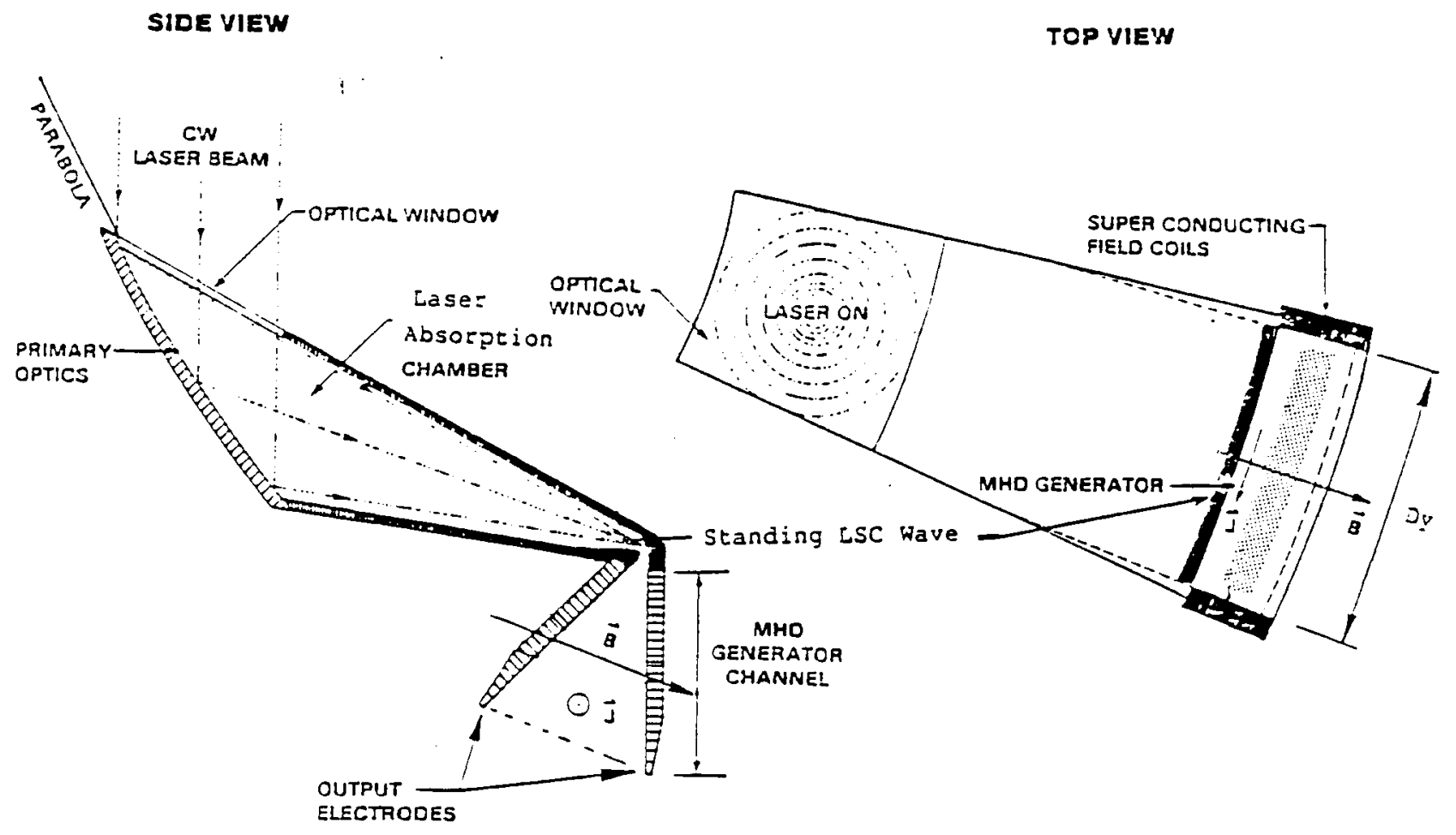

Figure 33. Concept for Laser-heated MHD generator. 
to net power into the flow of $65 \%$. For the large static preseures and high velocities at the inlet of the MHD generator, and using highly reflective walls inside the absorption chamber, it is hoped that a conversion efficiency of $80 \%$ can be achieved.

A theoretical analysis of the absorption chamber for the Apollo Lightcraft was not performed; however, A. Setagesh of Tuskegee University is investigating hydrogen LSC waves in rectangular geometries similar to the inlet of the MHD generator. The laser energy per mass of propellant required to raise the temperature of the hydrogen entering the absorption chamber to the inlet temperature of the MHD generator is currently calculated by

$$
Q_{L}=\frac{1}{\eta_{c h}}\left(H O_{i}-H O_{c h}\right)
$$

where the stagnation enthalpies $\mathrm{HO}_{\mathrm{ch}}$ and $\mathrm{HO}_{i}$ are calculated at the entrance conditions of the laser absortion chamber and MHD generator respectively, and a conversion efficiency, $\eta_{c h}$, of $100 \%$ is used. A more realistic value for $\eta_{c h}$ will be used after an analysis of the absorption chamber has been completed. The liquid hydrogen, stored as para hydrogen, will be used to regeneratively cool the walls of the absorption chambers and MHD generators, which are assumed to support a constant wall temperature of $2500 \mathrm{~K}$.

\section{B. MHD GENBRATOR}

\section{Geometry}

The geometry of the MHD generator is shown in Fig. 34. The cross-sectional area of the generator duct is rectangular, with a constant width $D_{y}$ and a variable height $D_{z}$. The core flow $U_{c}$ is in the $\mathrm{x}$-direction, the applied magnetic field $\mathrm{B}$ is in the z-direction, and the induced Faraday current density, $J_{y}$, resulting from the flow of the ionized hydrogen perpendicular to the applied field $B$, is in the negative Y-direction. The electrode walls (the two walls perpendicular to the current flow $J_{y}$ ) are segmented. The external load across each pair of electrodes is such that the loading parameter, $\mathrm{K}$, remains constant along the generator duct. The ionized hydrogen exits into the ambient air aft of the vehicle contributing to the vehicle thrust.

\section{Theory}

The high pressures which exist throughout the generator allow the gas to be considered in local thermodynamic equilibrium (LTE); so, although the gas consists of several species, its physical properties may be described by a single temperature and pressure. Also, for the conditions encountered in the MHD generator, the gas as a whole may be treated as a continuum. Thus, the flow may be adequately modelled by a single set of global continuum equations consisting of the 
conservation of mass, electrical charge, linear momentum, and energy, and a transport equation for the current density (usually referred to as the generalized Ohm's law in one of its simplified forms). To describe the electromagnetic fleld interaction in the gas, the Maxwell equations for the curl of the magnetic intensity vector $\vec{H}$ and the curl of the electric field vector $\vec{E}$, along with their constitutive equations, are required in general. For gaseous mediums, the relative permeability $\mu$ and permittivity $\epsilon$ of the gas are approximately equal to their free space values of $\mu_{0}$ and $\epsilon_{0}$.

The Debye length may be interpreted as the distance by which electrons and ions will separate in a ionized gas due to the thermal velocity of the electrons. The Debye length (in S.I. units) is defined by

$$
h_{D}=\left(\frac{\epsilon_{0} k T}{n_{e} e^{2}}\right)^{\frac{1}{2}}=69 \sqrt{T / n_{e}}
$$

where $T$ is the gas temperature and $n_{e}$ is the electron number density. The dimensions of the generators considered for the Apollo Lightcraft are on the order of centimeters (or more), while the maximum Debye length typically encountered is on the order of 0.1 micrometers; thus, the gas is essentially electrically neutral throughout the generator.

For many MHD generators, it is often true that the average of the applied field over the crosssectional area of the duct is relatively undistorted by the internally produced magnetic fields resulting from the electrical currents within the gas. The Maxwell equation for the magnetic induction, $\vec{B}$, consistent with the assumptions of zero net charge density (and therefore the diplacement vector $\vec{D}$ is negligible) and a relative permeability of $\mu_{0}$ is given by

$$
\vec{\nabla} \times \vec{B}=\mu_{0} \vec{J}
$$

An order of magnitude approximation of this equation leads to a parameter describing the distortion of the applied magnetic fleld given by

$$
\frac{d B}{B}=\frac{\mu_{0} J_{y} D_{z} / 2}{B}
$$

where $J_{y}$ and $D_{z} / 2$ are characteristic values for the current density perpendicular to the applied magnetic field $B$ and the length over which the magnetic field acts, and $\mathrm{dB}$ is a measure of the distortion or change in the magnetic fleld due the internal currents $J_{y}$. As shown in Fig. 35, the parameter $d B / B$ is approximately equal to the tangent of the angle through which the applied field is deflected by the internal magnetic fields. It is assumed in this analysis that for $d B / B$ less than $1 / 2$, the distortion of the applied magnetic field is negligible. With this assumption, the magnetic field distribution becomes a known quantity and the Maxwell equation (3) is not needed.

The transport equation for the current density may be derived by considering the first moment of the Boltzmann equation for each species in the gas. This leads to a highly nonlinear 


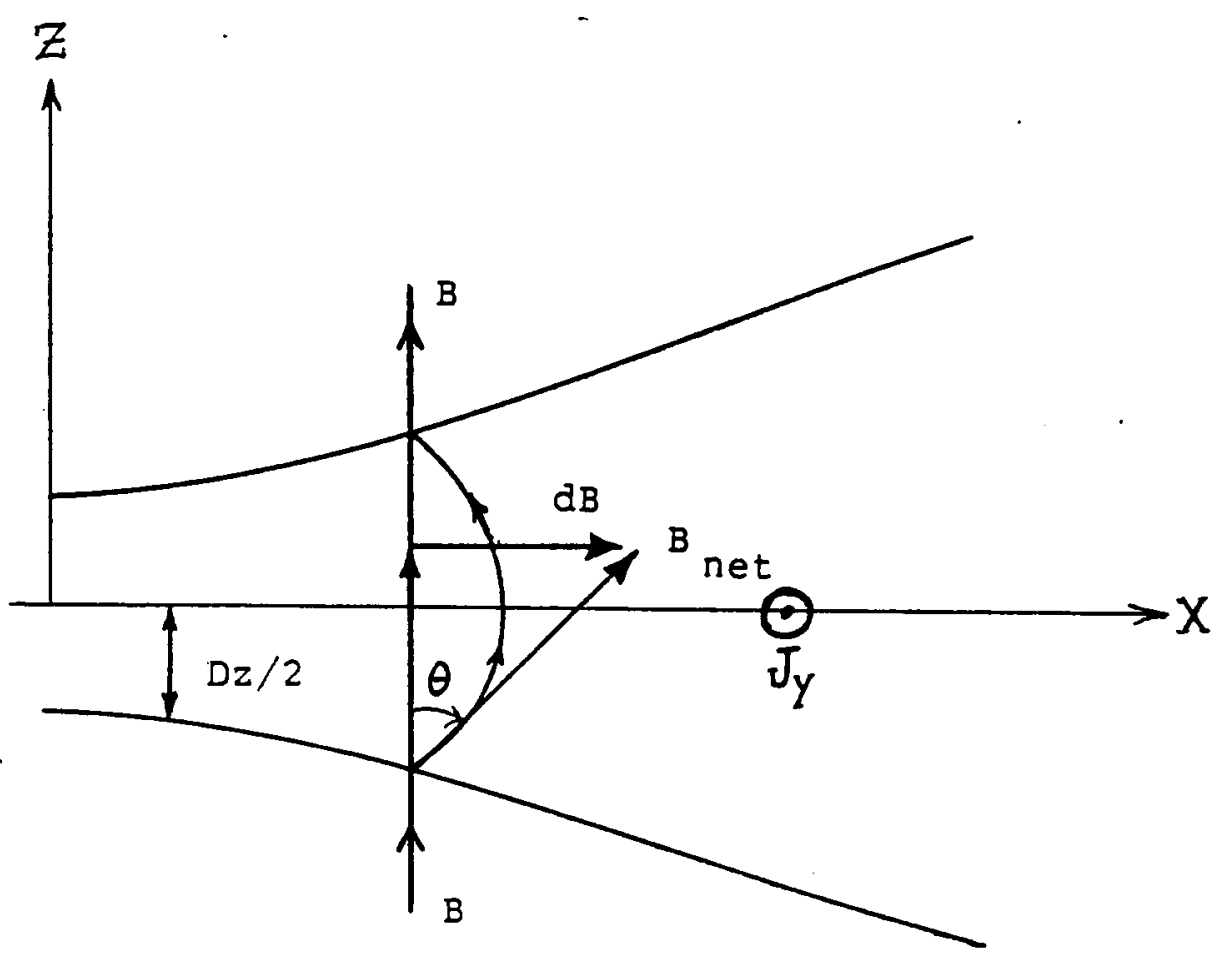

Figure 35. Distortion of the applied magnetic field in the MHD generator. 
partial differential equation for the current denvity vector $\vec{J}$. However, for the MHD generator operating conditions, many of the terms are negligible and the transport equation for the current density or the generalized Ohm's law becomes

$$
\vec{J}=\sigma(\vec{E}+\vec{V} \times \vec{B})-\frac{\omega_{e} \tau_{e}}{\vec{B}}(\vec{J} \times \vec{B})
$$

where the conductivty $\sigma$ accounts for both electron-ion collisions and electron-neutral collisions, and the Hall parameter $\omega_{e} \tau_{e}$ represents the ratio of the cyclotron frequency of the electrons to the collision frequency of the electrons.

Also, the gas flow is assumed to be steady and will almost certainly be turbulent in any real application, especially for the high velocities typical of the generators considered here. The radiation stress tensor and internal energy, which must in general be included in the momentum and energy equations, can be shown to be negligible based on order of magnitude calculations for a black gas at typical generator temperatures $(10,000$ to $20,000 \mathrm{~K}$ in this paper). The radiative heat flux, however, is quite significant and will eventually be considered in the analysis; however, in the present analysis, radiative heat transfer between the hydrogen gas and its surroundings will be neglected.

To simplify the analysis, the governing equations are integrated or averaged over the crosssectional area of the generator duct. The applied magnetic field $B$ is a function of the axial coordinate $x$ only (an axially decreasing field is used) and is in the z-direction as indicated previously. The shear stress and heat flux are assumed uniform around the perimeter of the generator duct at a given $x$ location, and the density and pressure are assumed to be functions of $x$ only. The electrode walls are assumed to be infinitely segmented so that no Hall current $J_{3}$ can flow, although a Hall electric field $E_{x}$ will be present. Finally, making some assumptions consistent the turbulent and boundary layer nature of the fiow, the integration of the equations over the cross-sectional area of the generator yields the following set of quasi-one-dimensional MHD equations:

$$
\begin{array}{cc}
\text { Continuity : } \rho \imath A=\dot{m} & \\
\text { or } & (I V-6) \\
\frac{d \rho}{\rho}+\frac{d u}{\imath}+\frac{d A}{A}=0 & (I V-7) \\
\text { Momentum : } \rho \llbracket \frac{d q}{d x}+\frac{d P}{d x}=-\left(\tau_{w} \frac{\rho}{A}+J_{y} B\right) & (I V-8) \\
\text { Energy : } \rho \imath \frac{d \hbar}{d x}+\rho \eta^{2} \frac{d q}{d x}=-\left(g_{w} \frac{\rho}{A}+J_{y} E_{y}\right) &
\end{array}
$$




$$
\begin{aligned}
& \text { Generalized Ohm's Law : } J_{y}=\frac{\sigma}{G}\left(a B-E_{y}\right) \quad(I V-9) \\
& \text { Non - uniformity factor : } G=\overline{\sigma\left(\frac{\Gamma}{\sigma}\right)}+\left(\omega_{e} \tau_{e}\right)^{2}\left[\overline{\sigma\left(\frac{T}{\sigma}\right)}-1 \mid \quad(I V-10)\right. \\
& \text { Gas Law }: \rho=\frac{P}{R_{0} T}<M W>\quad(I V-11) \\
& \text { Loading parameter: } K=\frac{E_{y}}{a B} \quad(I V-12)
\end{aligned}
$$

Expressions for the wall shear stress $\tau_{w}$ and the convective heat flux flowing into the wall, $q_{w}$, were obtained by approximating the flow along each wall of the generator as a steady, compressible, turbulent flat plate boundary layer flow. The friction coefficient, $C_{f}$, was calculated using Christoph's and White's inner variable method applied to a turbulent flat plate boundary layer flow [17], while the Stanton number was calculated using the Reynolds analogy,

$$
S t=\frac{1}{2} C_{f}\left(P r^{*}\right)^{2 / s}
$$

The wall shear stress and convective heat transfer are given by

$$
\begin{gathered}
T_{w}=\frac{1}{2} \rho_{c} U_{c}^{2} C_{f} \\
q_{v}=\rho U_{c}\left(h_{c}+\frac{U_{c}^{2}}{2}-h_{v}\right) S t
\end{gathered}
$$

The mole fraction for each species in the hydrogen plasma at a given temperature and pressure was found by applying the law of mass action to the following reactions only :

$$
\begin{gathered}
H_{2} \rightleftharpoons 2 H \\
H \rightleftharpoons H^{+}+e^{-}
\end{gathered}
$$

With the mole fractions known, the density is calculated using a equation (11), where $\langle M W\rangle$ is the average molecular weight of the mixture given by

$$
<M W>=\sum_{i} \chi_{i} M W_{i}
$$

Although the composition of the hydrogen predicted by the above analysis neglects several important species and differs significantly with Patch's data [11], whose model considered spin-equilibrated 
hydrogen in chemical equilibrium in the Debye - Huckel approximation and accounts for the opecies $\mathrm{H}^{-}, \mathrm{H}_{2}^{+}$, and $\mathrm{H}_{3}^{+}$, values for the average molecular weight and the electron mole fraction (important for the calcuation of conductivity) are within $10 \%$ or better.

The enthalpy as a function of temperature and pressure was calculated from a curve fit of Patch's data, whose values are within $10 \%$.

The conductivity $\sigma$ appearing in the generalized Ohm's law is given by the following equation

$$
\sigma=\frac{n_{e} e^{2}}{m_{e} C_{e}}\left[\frac{1}{\sum n_{k} Q_{k}+3.9 n_{e}\left(\frac{e^{2}}{8 \pi \epsilon_{0} K T}\right)^{2} \ln \Lambda}\right]
$$

where $Q_{k}$ is the collision cross section of the neutral species $k$ for momentum transfer with an electron, $C_{\mathrm{o}}$ is the mean random velocity assuming a Maxwell-Boltzmann distribution for the electrons, and $\Lambda$ is the ratio of the Debye shielding length to the impact parameter for $90^{\circ}$ Coulomb scattering.

The viscocity of the gas was approximated by a curve fit of data obtained by Yost [19].

\section{Generator Design}

Equations (6) - (8) represent a set of coupled, nonlinear, first order differential equations, which can be expressed, at least implicitly, in terms of $T, \mathrm{P}, \boldsymbol{a}, D_{z}, D_{y}$ and $\mathrm{x}$. Typically, one would consider $\mathrm{x}$ as the independent variable for some prescribed area distribution, $A=D_{y} D_{x}(x)$. However, in order to design a generator for the Apollo Lightcraft, it is advantageous to leave the area distribution variable (but with a specifled shape, ie., a rectangular cross section) and specify some other condition to close the set of equations, since the volume of the generator can greatly effect its efficiency and power output, while its surface area will effect the heat and friction losses at the walls of the generator. The condition imposed in this analysis is that of a constant velocity, $u$, throughout the generator. This simplifies the solution of the set of differential equations describing the generator, but it also removes the threat of flow separation, a problem which may occur in a flow with a decreasing velocity.

The solution to this set of differential equations was performed by approximating each differential in Eqns. (6) - (8) by a forward difference:

$$
\frac{d f(x)}{d x} \approx \frac{f(x+\Delta x)-f(x)}{\Delta x}
$$

The differential of the enthalpy can be written as

$$
d h=\left(\frac{\partial h}{\partial T}\right)_{P} d T+\left(\frac{\partial h}{\partial P}\right)_{T} d P=C_{P} d T+C_{T} d P
$$

$C_{p}$ and $C_{T}$ were also approximated by forward differences. Using $T$ as the marching variable, Eqns. (6) - (8) can be solved to yield:

$$
\Delta x=\frac{-C_{P} \Delta T}{\left(J_{y} E_{y}+q_{w} \frac{\rho}{\lambda}\right) \frac{A}{\hbar}-C_{T}\left(J_{y} B+\tau_{w} \frac{p}{A}\right)}
$$




$$
\Delta P=-\left(J_{y} B+\tau_{w} \frac{P}{A}\right) \Delta x
$$

For a given set of initial conditions, we calculate all the necessary variables, let $T$ increase by some $\Delta T$ (ie., we march in $T$ ), and calculate the change in $x$ and $P$ using Eqns. (20) and (21). $\rho$ and $D_{z}$ are updated according to

$$
\begin{gathered}
\rho(x+\Delta x)=\rho(T(x)+\Delta T, P(x)+\Delta P) \\
D_{z}=\frac{\dot{m}}{\rho u D_{y}}
\end{gathered}
$$

In the above equations and in any futher references, the bar superscript over averaged quantities will be left out for convenience. Also, at each step, the electrical work extracted per unit mass, $\Delta w$, and the convective heat loss to the walls per unit mass, $\Delta(\mathrm{QT})$, are calculated by

$$
\begin{gathered}
\Delta(Q T)=q_{w}(x) \frac{p \Delta x}{\dot{m}} \\
\Delta w=h(x)-h(x+\Delta x)-\Delta(Q T)
\end{gathered}
$$

The initial conditions for the generator consist of the entrance temperature, pressure, Mach number, and magnetic field, the loading parameter, and the mass flow rate. The parameter $d B / B$ and the velocity boundary layer thickness (assumed the same for each wall) are also calculated at each step through the generator.

Preliminary trajectory calculations indicated that a specific electric work of about $700 \mathrm{MJ} / \mathrm{kg}$ would be necessary if the Apollo Lightcraft was to achieve low Earth orbit and deorbit using the design point of $330 \mathrm{~kg}$ of hydrogen propellant or less. Also, the size of the generator was limited to a maximaum axial length of $\mathrm{L}=0.5 \mathrm{~m}$ to meet with the design point volume for the Apollo Lightcraft. As depicted in Fig. 39 (and in the Appendix), the vehicle will contain twelve (12) MHD generators which extend out during the MHD fanjet mode. Since the exterior wall in which the generators are contained must be parallel with the shroud, the ratio $L / D_{e}$ (where $D_{e}$ is the exit height of the generator) must be greater than 1.5. In order to maintain the generator walls at a temperature of $2500 \mathrm{~K}$, using only the $330 \mathrm{~kg}$ of liquid para hydrogen to cool the walls of the generators and the laser absorption chambers, the total convective heat/mass into the generator walls, QT, must be less than some maximum heat/mass, $Q T_{\text {mas. }}$. It is also desirable to have a high conversion efficiency of laser energy into total generated electrical energy, $w($ tot $) / Q_{L}$.

The design of the generator for the Apollo Lightcraft was accomplished by structuring the above into the following optimization problem : 
MAXIMIZE the objective function $f=w($ tot $)$ Subject to :

$$
\begin{aligned}
w & \geq 700 \mathrm{MJ} / \mathrm{kg} \\
L & \leq 0.5 \mathrm{~m} \\
L / D_{0} & \geq 2 \\
Q T & \leq Q T_{\operatorname{mes}} \\
w(\text { tot }) / Q_{L} & \geq 0.5 \\
P e & \geq 0.0108 \text { bars }
\end{aligned}
$$

plus

$$
\begin{aligned}
& d B / B \leq 0.5 \\
& 2 \delta / D_{z} \leq 1 \\
& 2 \delta / D_{y} \leq 1
\end{aligned}
$$

which must be satisfled throughout the duct.

It should be noted that large ratios of $L / D_{0}$ help reduce losses at the generator exit due to eddy current loops. The constraint on the exit pressure is necessary for a generator with subsonic flow at the exit to function at a minimum altitude of $100,000 \mathrm{ft}$. If the flow becomes supersonic within the generator and the exit pressure is less than the ambient pressure, a shock wave may form in the generator, which would presumably degrade generator performance. The last three constraints are imposed to ensure the validity of the theoretical model described previously for the analysis of the generator.

A generalized reduced gradient (GRG) code (OPT 3.1, Gabriel and Beltracchi [5]) was used to solve the above optimization problem. The design was attemped for two different expressions for $Q T_{\text {max }}$. In what will be referred to as the HIGH case, $Q T_{\max }$ was set to a constant value of $28 \mathrm{MJ} / \mathrm{kg}$. We can place a maximum value on $Q T_{\max }$ for a given storage temperature of $20 \mathrm{~K}$ for the liquid hydrogen by computing the heat/mass that could be absorbed by the hydrogen if it was heated to $2500 \mathrm{~K}$; assuming a constant $C_{p}$ of $15,000 \mathrm{~J} / \mathrm{kg} / \mathrm{K}, Q T_{\max }$ would be approximately 37 $\mathrm{MJ} / \mathrm{kg}$. The above value of $28 \mathrm{MJ} / \mathrm{kg}$ was used since the laser absorption chambers must also be cooled.

In what will be referred to as the LOW case, the value for $Q T_{\text {mex }}$ was computed assuming the cold hydrogen flowed through a simple cooling duct $1 \mathrm{~mm}$ in height along each wall of the generator and assuming a certain amount of heat loss in the laser absorption chamber estimated from a mean beam length calculation. In this case, $Q T_{\text {mex }}$ depended on the geometry of the generator and the 
temperature and pressure of the hydrogen at the generator inlet, and thus a separate subroutine for calculating $Q T_{\max }$ was incorporated into the previously described generator code. Typically, $Q T_{\text {mas }}$ was on the order of 2 to $6 \mathrm{MJ} / \mathrm{kg}$. This case is considered a lower bound on $Q T_{\text {mex }}$ since transpiration cooling or other advanced heat transfer methods would allow higher values for $Q T_{\text {max }}$.

\section{Results}

The resulting designs for the HIGH and LOW generators are described in Fig.'s 36 and 37. A feasible design point satisfying $w(t o t)=700 \mathrm{MJ} / \mathrm{kg}$ could not be found for the other constraints imposed on the Apollo Lightcraft design; thus, the optimization problem was simply to minimize the constraint violations. In both the HIGH and LOW cases, the constraints on the generator length $L$ and total heat loss to the walls QT are active (ie., essentially equal to zero), while all other constraints (except $w(t o t) \geq 700 \mathrm{MJ} / \mathrm{kg}$ ) were satisfled. The HIGH generator nearly satisfies the speciflc work goal of $700 \mathrm{MJ} / \mathrm{kg}$, producing $652.5 \mathrm{MJ} / \mathrm{kg}$; as will be discussed in the trajectory analysis (Chapter VI), this generator provides sufficient work/mass for the Lightcraft to achieve low Earth orbit and deorbit using no more hydrogen propellant than the design point of $330 \mathrm{~kg}$. The LOW generator can only produce a specific work of $362 \mathrm{MJ} / \mathrm{kg}$ in order to satisfy the tighter heat loss constraint; this would require over $330 \mathrm{~kg}$ of hydrogen to achieve low Earth orbit and deorbit (discussed further in Chapter VI). The two designs attempt to provide an upper and lower bound on the generator performance possible for the Apollo Lightcraft: $652.5 \mathrm{MJ} / \mathrm{kg}$ and $362 \mathrm{MJ} / \mathrm{kg}$ electric work/mass respectively.

\section{Generator Thrust}

Consider the single generator shown in Fig. 38. Ignoring the contribution to thrust from the expansion of the exhausted hydrogen over the plug nozzle (to ambient pressure), the generator thrust was calculated as

$$
\begin{gathered}
\tau_{\text {gen }}=\left[\dot{m} u+\left(P_{e}-P_{a}\right) A_{e}\right] \cos \beta \\
\beta=90^{\circ}-\left(45^{\circ}+\theta\right) \\
\theta=\tan ^{-1}\left(\frac{D_{e}-D_{i}}{2 L}\right)
\end{gathered}
$$

\section{MHD ACCGLGRATOR}

The accelerator duct is an annular channel formed between the shroud and the exterior walls of the MHD generators, which are located symmetrically about the base of the vehicle, as shown in Figs. 39 and 40. In the MHD-fanjet mode, the shroud will be in the most rear-ward position so 

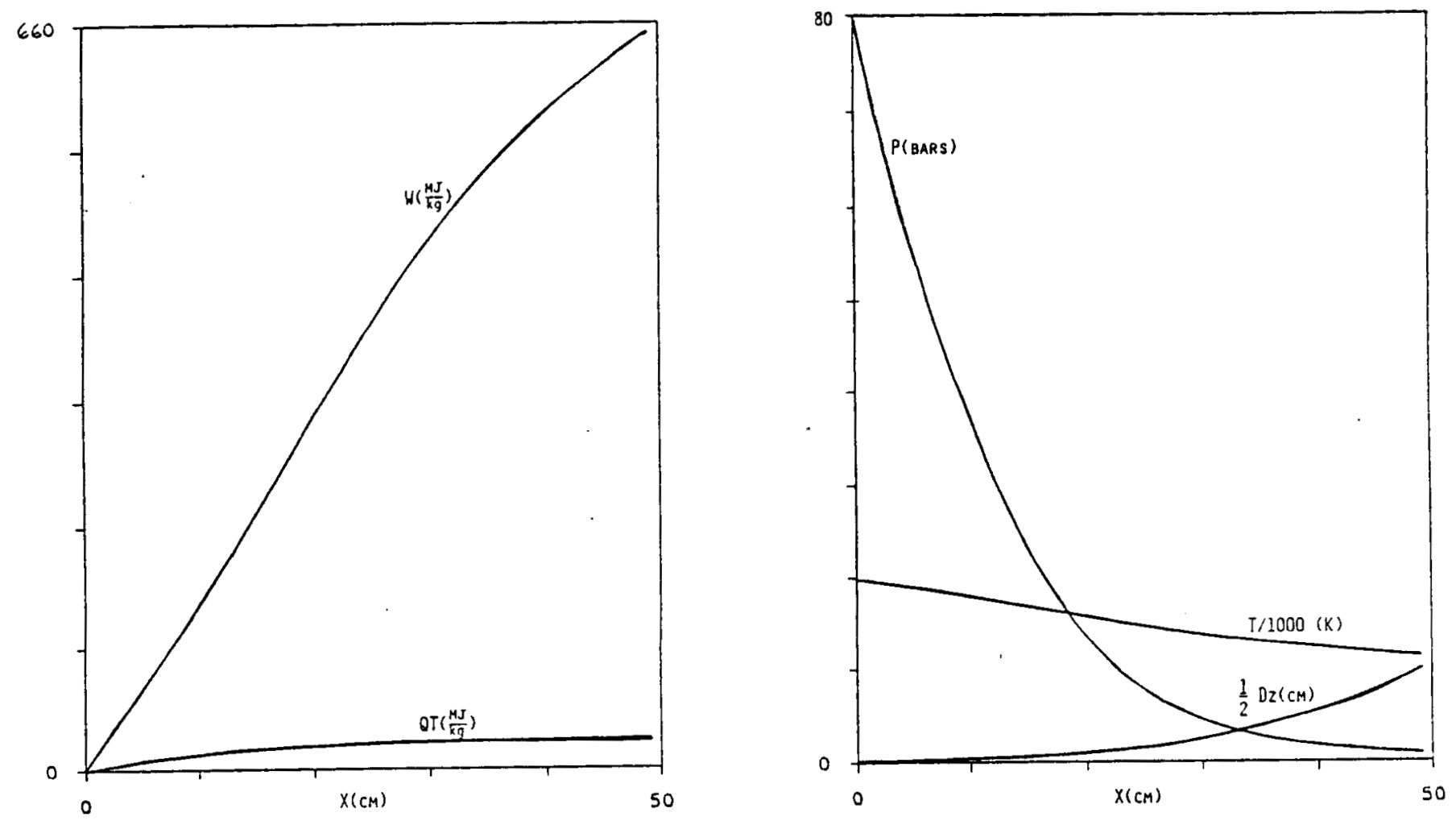

\begin{tabular}{|c|c|c|c|c|c|}
\hline $\begin{array}{l}T=2 \\
P=8 \\
B= \\
M=\end{array}$ & $\begin{array}{l}.000 \mathrm{~K} \\
.000 \text { bars } \\
.740 \text { tesia } \\
.534\end{array}$ & $\begin{array}{l}\dot{\mathrm{m}}= \\
\mathrm{K}= \\
\mathrm{Dy}= \\
\overrightarrow{\mathrm{u}}=\end{array}$ & $\begin{array}{r}0.4850 \\
0.9898 \\
33.0000 \\
8104.40\end{array}$ & $\begin{array}{l}\mathrm{kg} / \mathrm{s} \\
\mathrm{cm} \\
\mathrm{m} / \mathrm{s}\end{array}$ & \\
\hline$x(c m)$ & $\mathrm{Dz} / 2(\mathrm{~cm})$ & $T / 1000$ & PG (bars) & $\operatorname{QT}(\mathrm{MJ} / \mathrm{kg})$ & $W(M J / k g)$ \\
\hline $\begin{array}{r}0.00 \\
4.43 \\
8.76 \\
12.90 \\
16.88 \\
20.82 \\
24.92 \\
29.44 \\
34.75 \\
41.21 \\
49.10\end{array}$ & $\begin{array}{r}0.24 \\
0.31 \\
0.41 \\
0.56 \\
0.78 \\
1.12 \\
1.64 \\
2.48 \\
3.86 \\
6.18 \\
10.16\end{array}$ & $\begin{array}{l}20.00 \\
19.14 \\
18.28 \\
17.42 \\
16.55 \\
15.69 \\
14.83 \\
13.97 \\
13.11 \\
12.25 \\
11.38\end{array}$ & $\begin{array}{r}80.00 \\
58.28 \\
41.38 \\
28.60 \\
19.22 \\
12.54 \\
7.92 \\
4.84 \\
2.86 \\
1.63 \\
0.90\end{array}$ & $\begin{array}{r}0.00 \\
7.90 \\
13.48 \\
17.34 \\
20.01 \\
21.92 \\
23.37 \\
24.55 \\
25.60 \\
26.59 \\
27.59\end{array}$ & $\begin{array}{r}0.00 \\
63.98 \\
129.23 \\
195.38 \\
262.11 \\
329.05 \\
395.88 \\
462.22 \\
527.58 \\
591.31 \\
652.51\end{array}$ \\
\hline \multicolumn{4}{|c|}{$\begin{array}{l}\text { Laser Energy required, } Q_{L} \\
\text { Maximum value of dB/B } \\
\text { Conversion efficiency, w }\end{array}$} & \multicolumn{2}{|c|}{$1.2364 \mathrm{GJ} / \mathrm{kg}$} \\
\hline
\end{tabular}

Figure 36. The HIGH case MHD generator. 

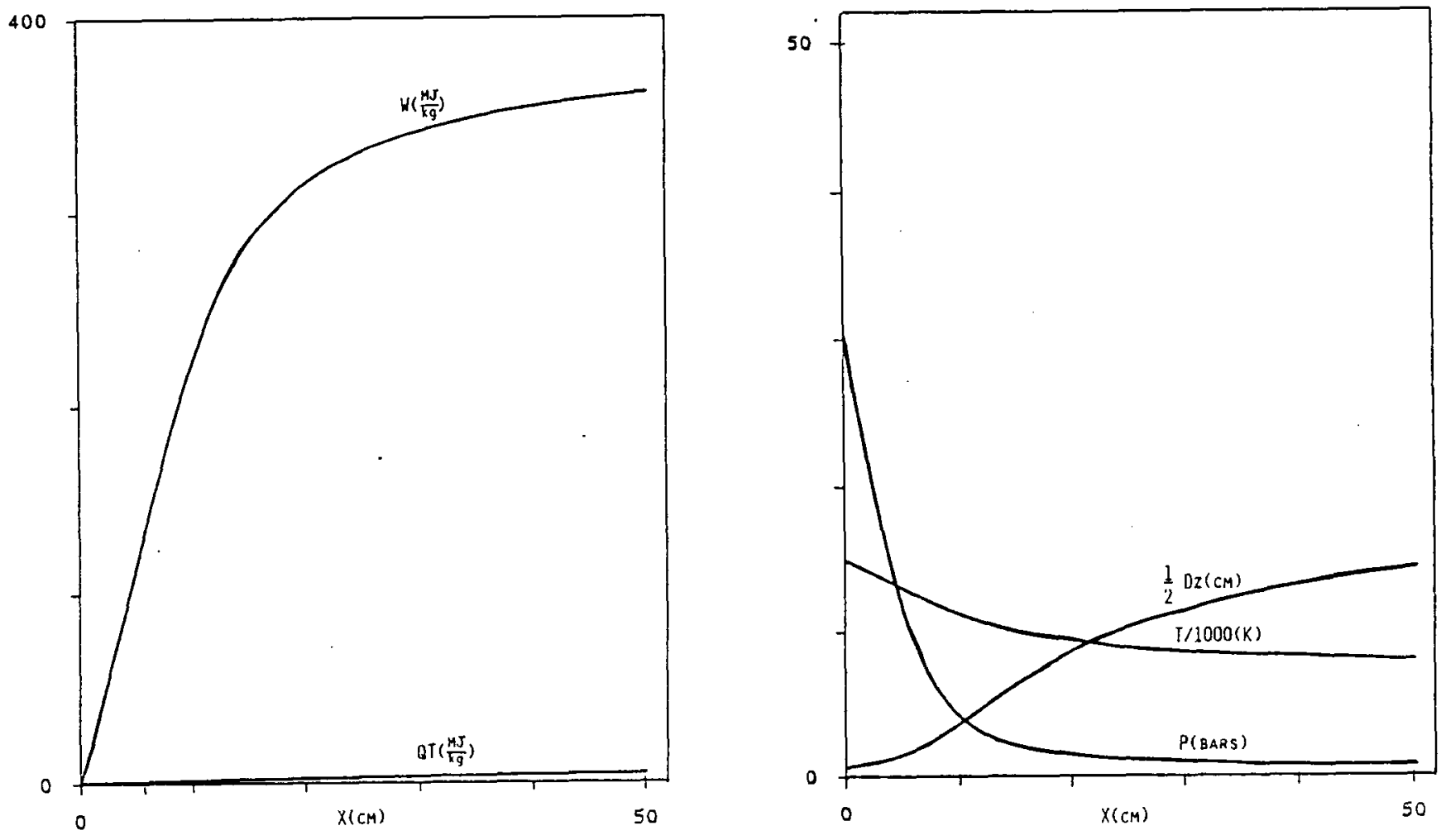

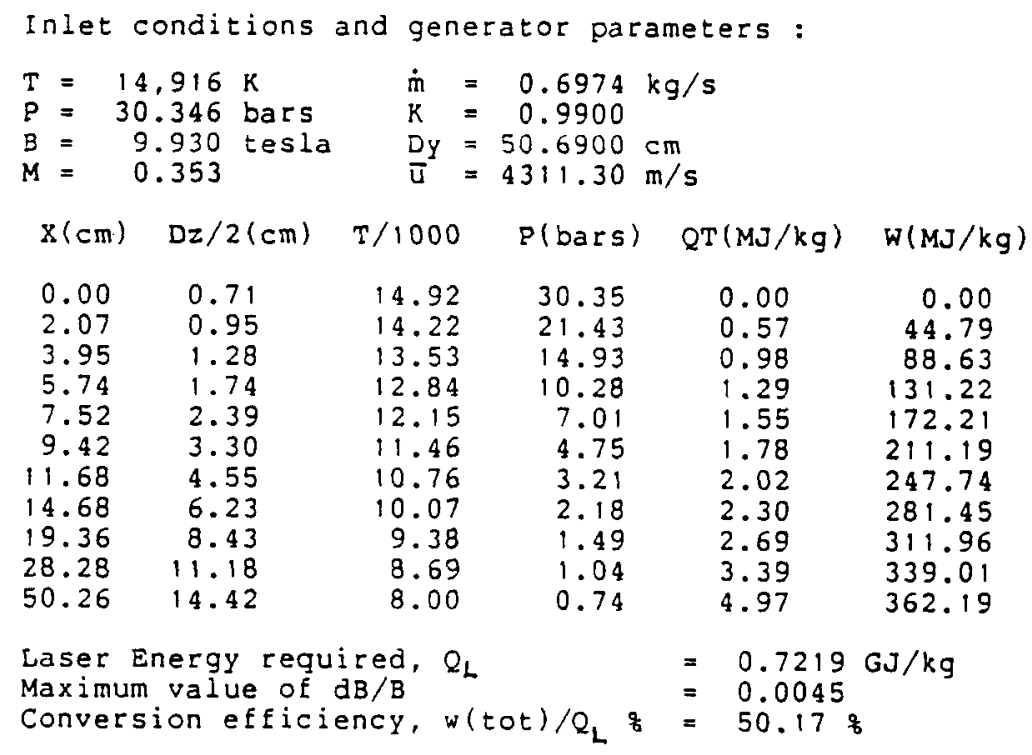

Figure 37. The LOW case MHD generator. 
that the forebody bow shock is attached at all times. For Mach numbers above 12, the position of the shock wave does not change significantly. The principle of operation for the MHD accelerator is unique in that energy is added to the flow through an electromagnetic fleld and converted directly to kinetic energy. For vehicles travelling in the hypersonic regime, the formation of a strong bow shock can result in static temperatures downstream of the shock on the order of several thousand degrees Kelvin. As a result, the air passing through this region is partially ionized and effective electrical conductivity is increased. This conductivity is then in general a function of altitude and Mach number, as well as bluntness of the vehicle forebody.

The fight envelope currently envisioned for the MHD fanjet mode begins roughly at Mach 12 at $100,000 \mathrm{ft}$. This approaches the limit of operation of the Scamjet. Obtaining analytical performance data for this engine involved an investigation of the flow over the conical forebody, and the subsequent acceleration in the MHD channel itself. Operation at the relevent hypersonic velocities required the use of real gas data both through the inlet and in the MHD accelerator.

\section{Theory}

The assumptions made for the hydrogen flow through the MHD generator also apply to the air flow through the MHD accelerator; thus, the quasi-one-dimensional equations (6) - (12) can be used. Some futher simplifications are made for the MHD accelerator analysis. The shear stress and heat transfer (both conductive and radiative) at the wall are neglected, as well the Hall term, $\vec{J} x \vec{B}$, in Ohm's law (5). Also, the differential of the enthalpy is represented by $\mathrm{dh}=C_{p} \mathrm{dT}$, where $C_{p}$ is considered a function of temperature and pressure. For the accelerator, the applied magnetic fleld and the cross-sectional area are constant throughout the accelerator channel. With these conditions and assumptions, the quasi-one-dimensional MHD equations for the MHD accelerator analysis become:

$$
\begin{array}{cc}
\text { Continuity : } \frac{d \rho}{\rho}+\frac{d u}{u}=0 & (I V-27) \\
\text { Momentum : } \rho u \frac{d u}{d x}+\frac{d P}{d x}=J_{y} B & (I V-28) \\
\text { Energy : } \rho u C_{P} \frac{d T}{d x}+\rho u^{2} \frac{d u}{d x}=J_{y} E_{y} & (I V-29) \\
\text { Ohm's Law : } J_{y}=\sigma\left(E_{y}-U B\right) & (I V-30) \\
\text { Equation of State : } R=\frac{R_{u}}{\langle M W\rangle} ; \rho=\frac{P}{R T} & (I V-31)
\end{array}
$$

The parameter $\mathrm{K}$ is still defined as $E_{y} / \mathrm{uB}$, but for the case of the accelerator is greater than 1 .

\section{Accelerator Model}

Re-arranging Eqns. (27) - (31) in terms of differentials for velocity, temperature, pressure, and density, using Ohm's law and the loading parameter to eliminate $J_{y}$ and $E_{y}$, and defining the 


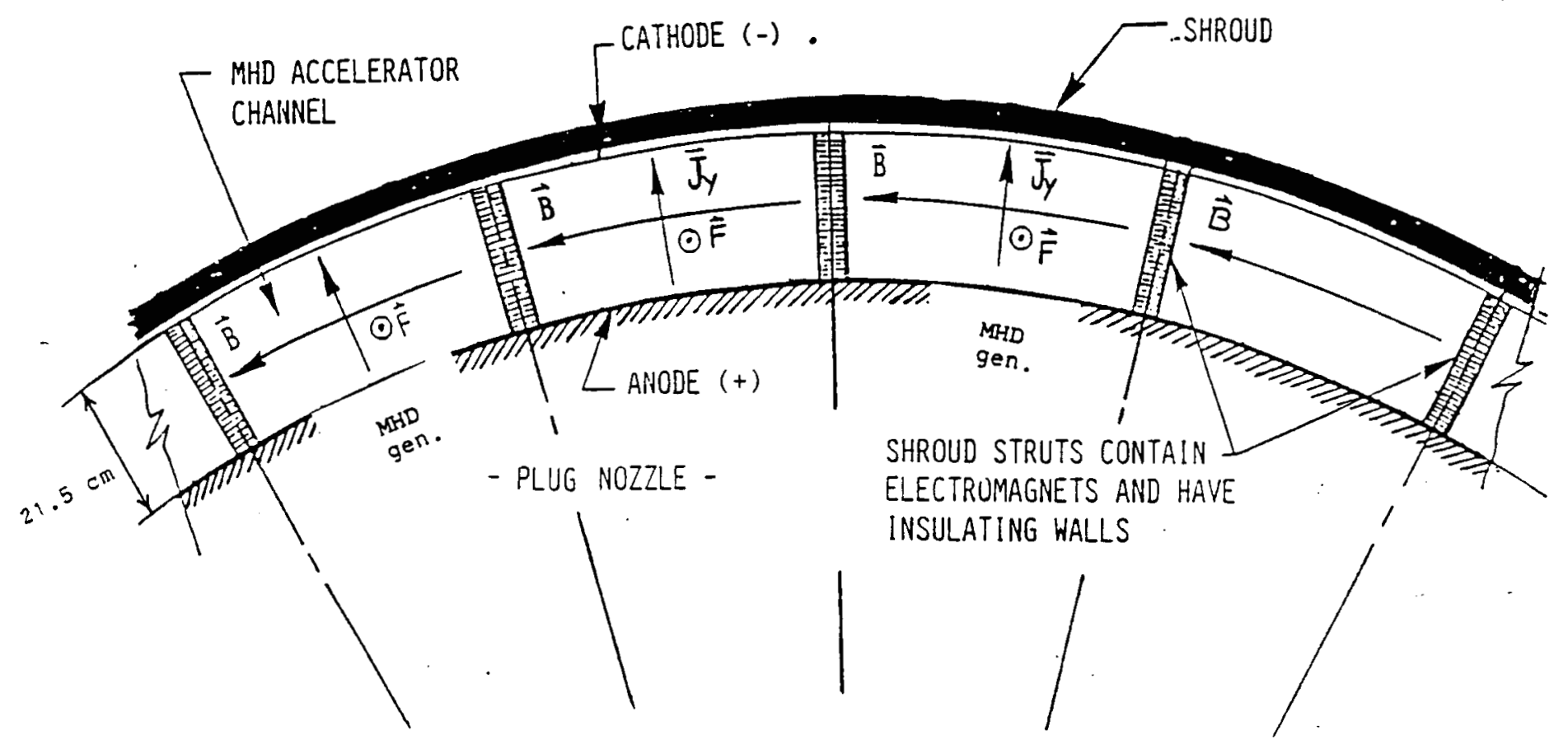

Figure 39. MHD accelerators and generators (rear view - looking forward)

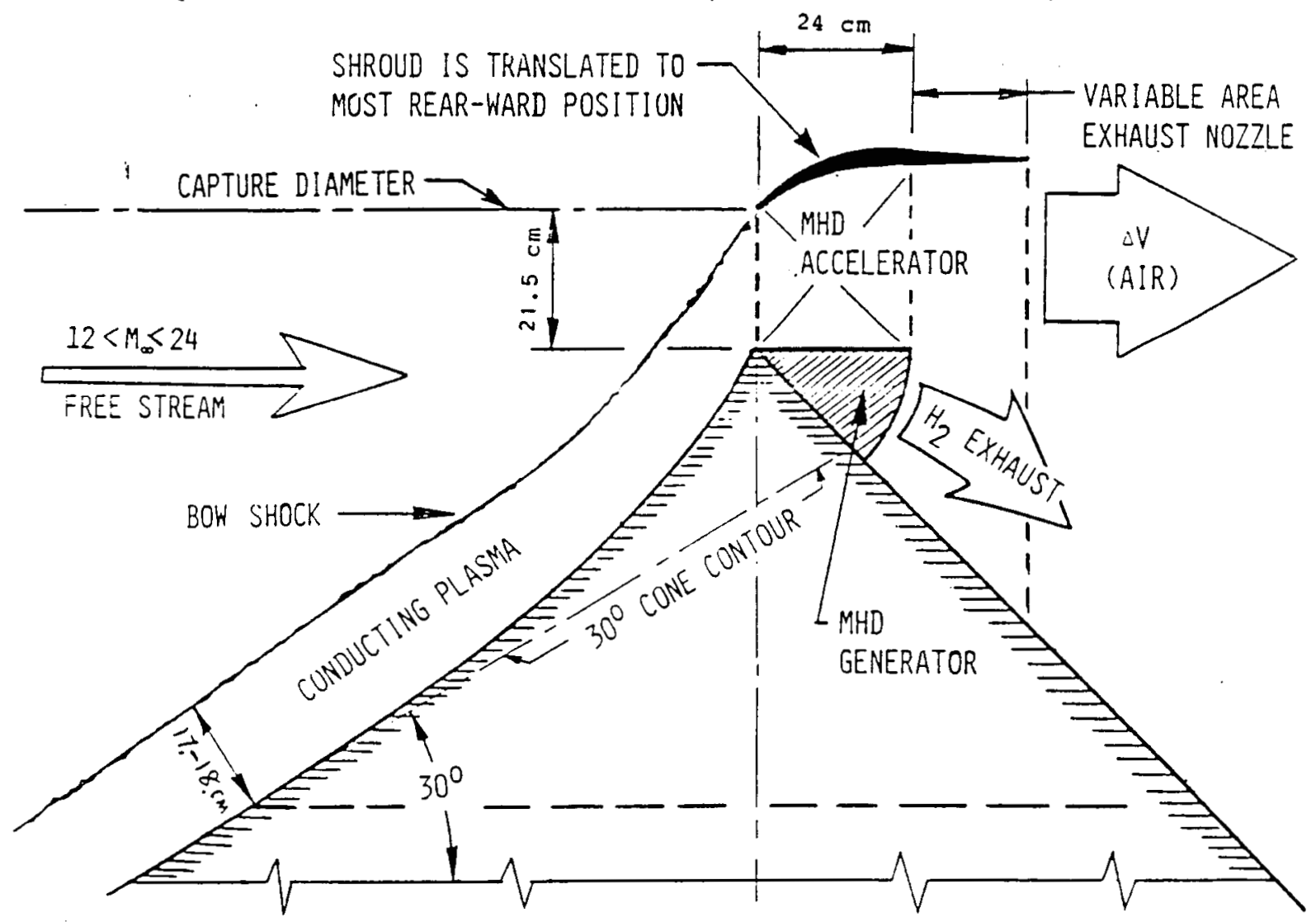

Figure 40. MHD accelerator and generator (side view) 
parameter $\mathrm{Z}$ as the mass flow rate per unit area (constant), gives the following set of relations:

$$
\begin{gathered}
\Delta u=\frac{\sigma u^{3} B^{2}(K-1)\left(1-\frac{K R}{C_{P}}\right)}{Z\left[u^{2}\left(1-\frac{R}{C_{P}}\right)-R T\right]} \Delta x \\
\Delta T=\frac{\sigma u^{2} B^{2} K(K-1) \Delta x}{Z C_{P}}-\frac{u \Delta u}{C_{P}} \\
\Delta P=\frac{Z R}{u}\left[\Delta T-\frac{T}{u} \Delta u\right] \\
\Delta \rho=\frac{1}{R T}\left[\Delta P-\frac{P \Delta T}{T}\right]
\end{gathered}
$$

These equations are readily integrated for prescribed values of $B$ and $K$, initial values of $u$, $T$ and $P$, and a step size $\Delta x$. An inlet analysis provided the required initial velocity and thermodynamic state variables, and a tabulation of the electrical conductivity, specific heat, and gas constant provided the necessary thermophysical properties.

Data for $C_{p}, \sigma$, and $\mathrm{R}$ for air at elevated temperatures over a wide range of pressures was obtained from data calculated by Yos [18]. While not tabulated explicitly, it was possible to calculate the value of the gas constant $\mathrm{R}$ from the given data for temperature, pressure, and density. These tables were then made available to a subroutine which would determine the required properties for the given temperature and pressure at each step of the solution. Fig. 41 shows a typical profile solution in non-dimensional form.

\section{Isothermal Solution}

Heretofore, nothing has been mentioned regarding the selection of the loading parameter $\mathrm{K}$, except that it is prescribed for a given solution. The applied magnetic field is held constant along the length of the accelerator for a particular solution. If the loading parameter is also held constant, the result is an electric fleld profile increasing in proportion to the velocity. More generally, it is possible to alter the shape of the velocity profile by varying the applied electric field. It should therefore be possible to select the electric field profile to optimize the velocity increase through the accelerator for a given magnetic field. This analysis was done by Drake [4], who uses techniques of variational calculus to show that the solution corresponding to minimum channel length is equivalent to the solution for maximum thrust per unit exit area. More importantly, the optimal solution is found to be very close to that for a duct of constant cross-sectional area, which is the case of interest in the present analygis.

Ideally, one would want all the applied electrical energy to be converted into kinetic energy. The implication is that the flow will be isothermal since no energy goes into heating the gas. This requirement then provides the means of selecting $\mathrm{K}$ (and ultimately $E_{y}$ ) as a function of $\mathrm{x}$ by 


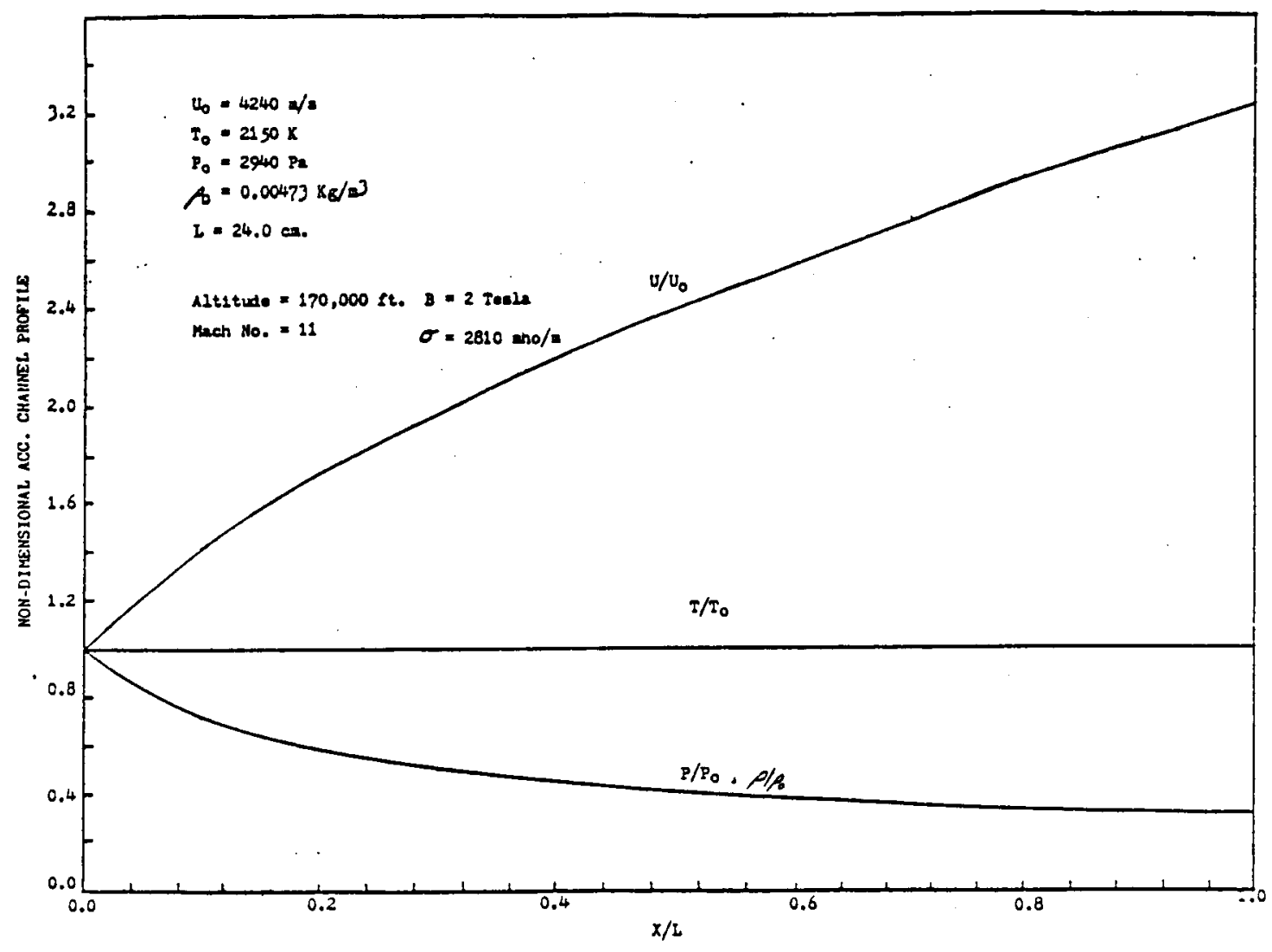

Figure 41. MHD accelerator profile of $u, T$ and $P$.

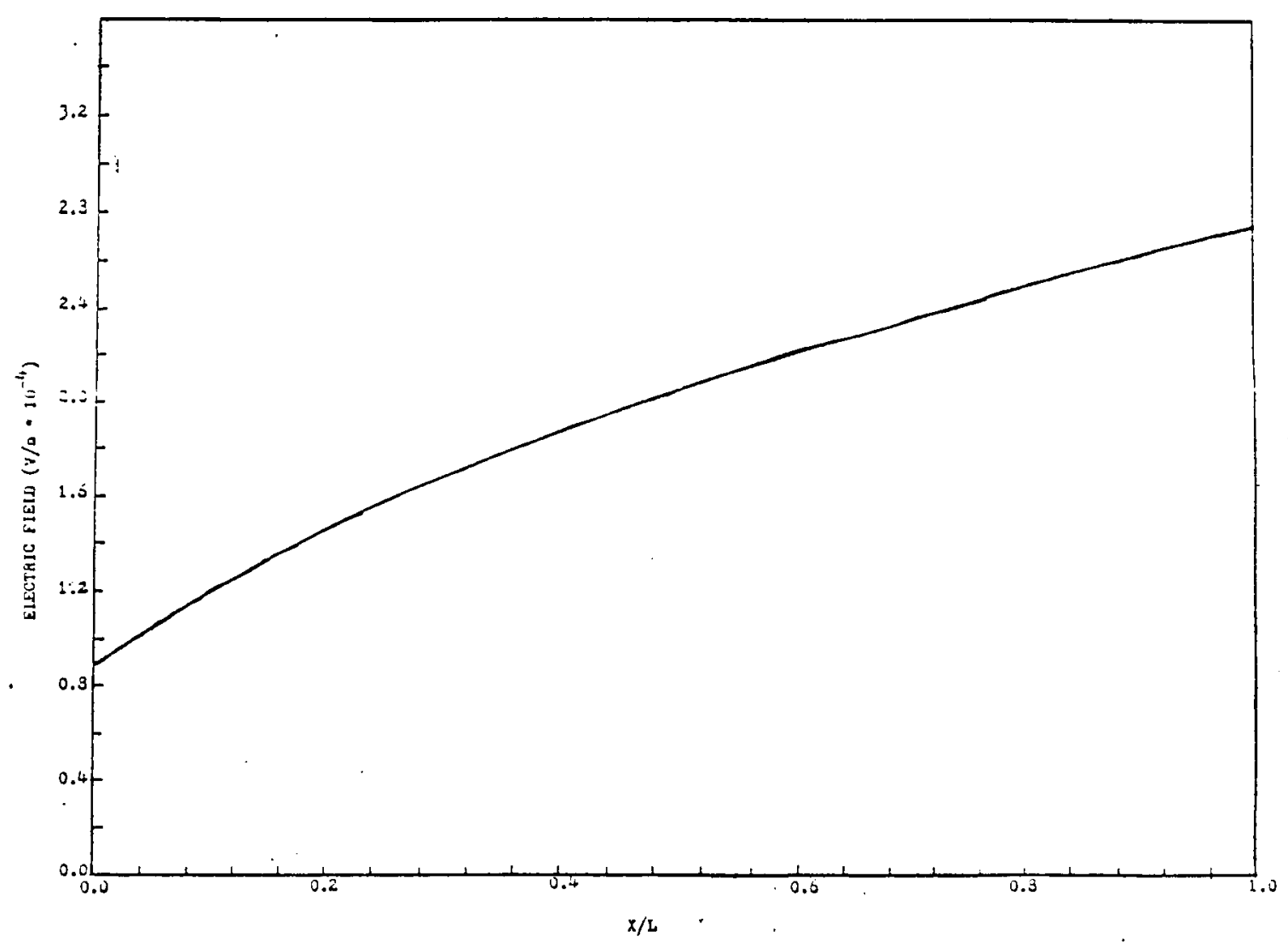

Figure 42. Electric field profile in accelerator. 
requiring the flow to be isothermal. This relation is readily obtained by setting $\Delta \mathrm{T}$ equal to zero in Eqn. (33), eliminating $\Delta u$ with Eqn. (32) and solving for $K$. This gives:

$$
K=\frac{1}{1-\frac{R T}{U^{2}}}
$$

which is the expression for $\mathrm{K}$, and hence $E_{y}$, to insure the temperature remains constant throughout the accelerator. The electric field proflle representative of the flow solution in Fig. 41 can be seen in Fig. 42.

As a practical matter, obtajning such a distribution along a constant area accelerator assumes the use of segmented electrodes, each at a different potential. Such segmenting, while perhaps difficult from the standpoint of insulating adjacent segments, is nevertheless a desirable feature to reduce hall currents.

\section{Inlet Analysis}

As was mentioned previously, before the numerical solution can be initiated, it is necessary to prescribe a set of initial conditions. These are obtained from analysis of the flow through the conical bow shock. For the range of Mach numbers under consideration ( $>12$ ), the static temperatuures even behind an oblique shock preclude the use of ideal gas relations for determining the temperature and pressure downstream of the shock. Oblique shock data for real gases can be found in the

literature [16] and curve fits based on this data were incorporated into the engine model to calculate temperature and pressure rises across the shock. With static temperature and pressure known it is possible to find the density using the equation of state and real gas properties for the given conditions. Finally the velocity is determined from the equation of continuity at the accelerator inlet.

The flow field about the conical inlet will be self similar with properties constant along conical surfaces swept out by rays emanating from the vertex. For the 1-D analysis, properties were assumed constant across the inlet plane of the accelerator. This is a reasonable assumption provided the channel height is small compared to the cone length.

\section{MHD-FANJET PERFORMANCE}

With the inlet and accelerator models established, it is possible to calculate the thrust, specific impulse, and coupling coefficient for the MHD-fanjet.

$$
\begin{array}{cc}
\tau=\dot{m}_{a}\left(U_{b}-U_{i}\right)+\left(P_{e}-P_{i}\right) A_{F}+\left(12 \tau_{g e n}\right) T H R O T & (I V-37) \\
I_{o p}=\frac{\tau}{\dot{m}_{j} g} & (I V-38)
\end{array}
$$




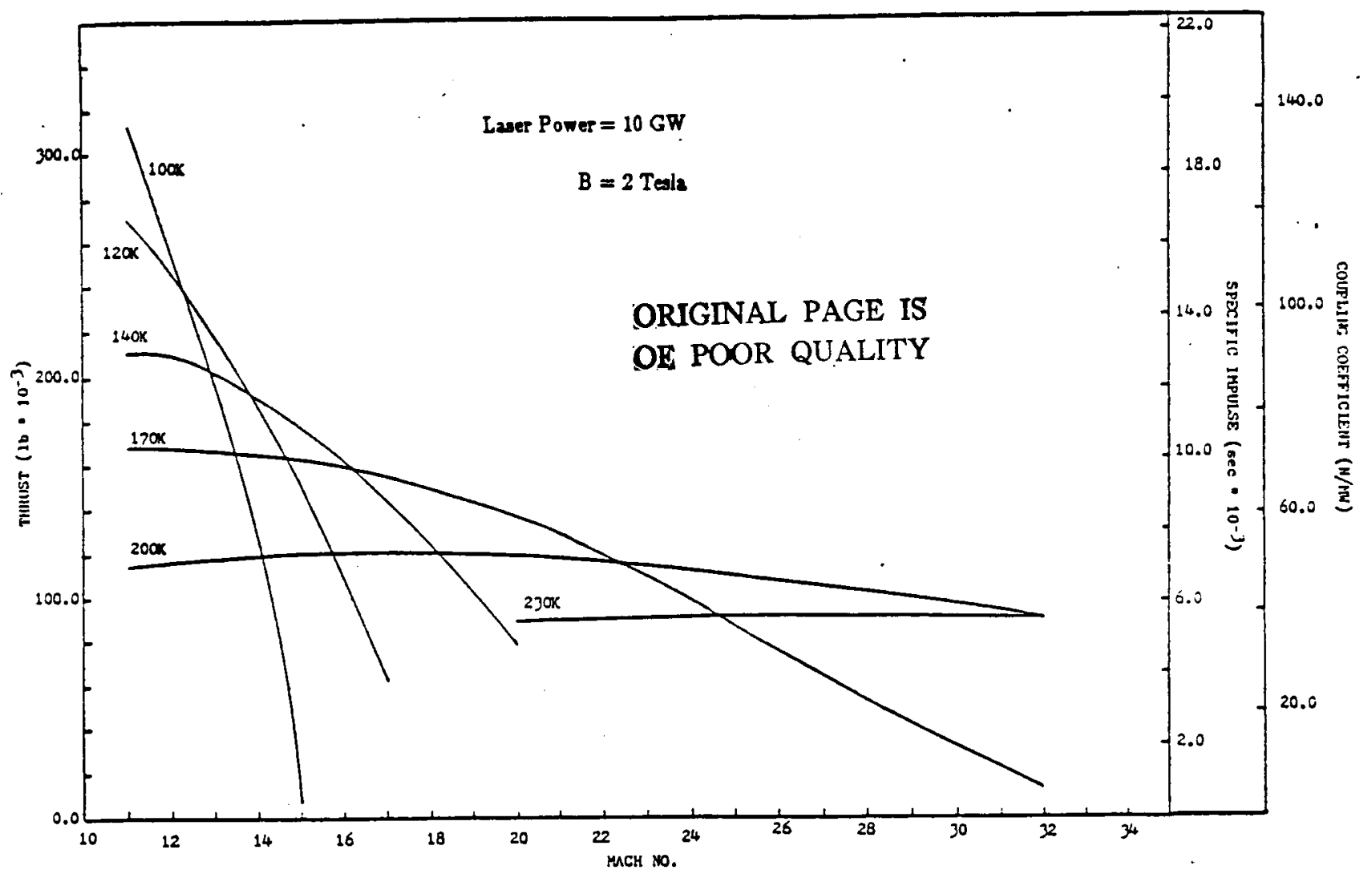

Figure 43. MHD-fanjet performance.

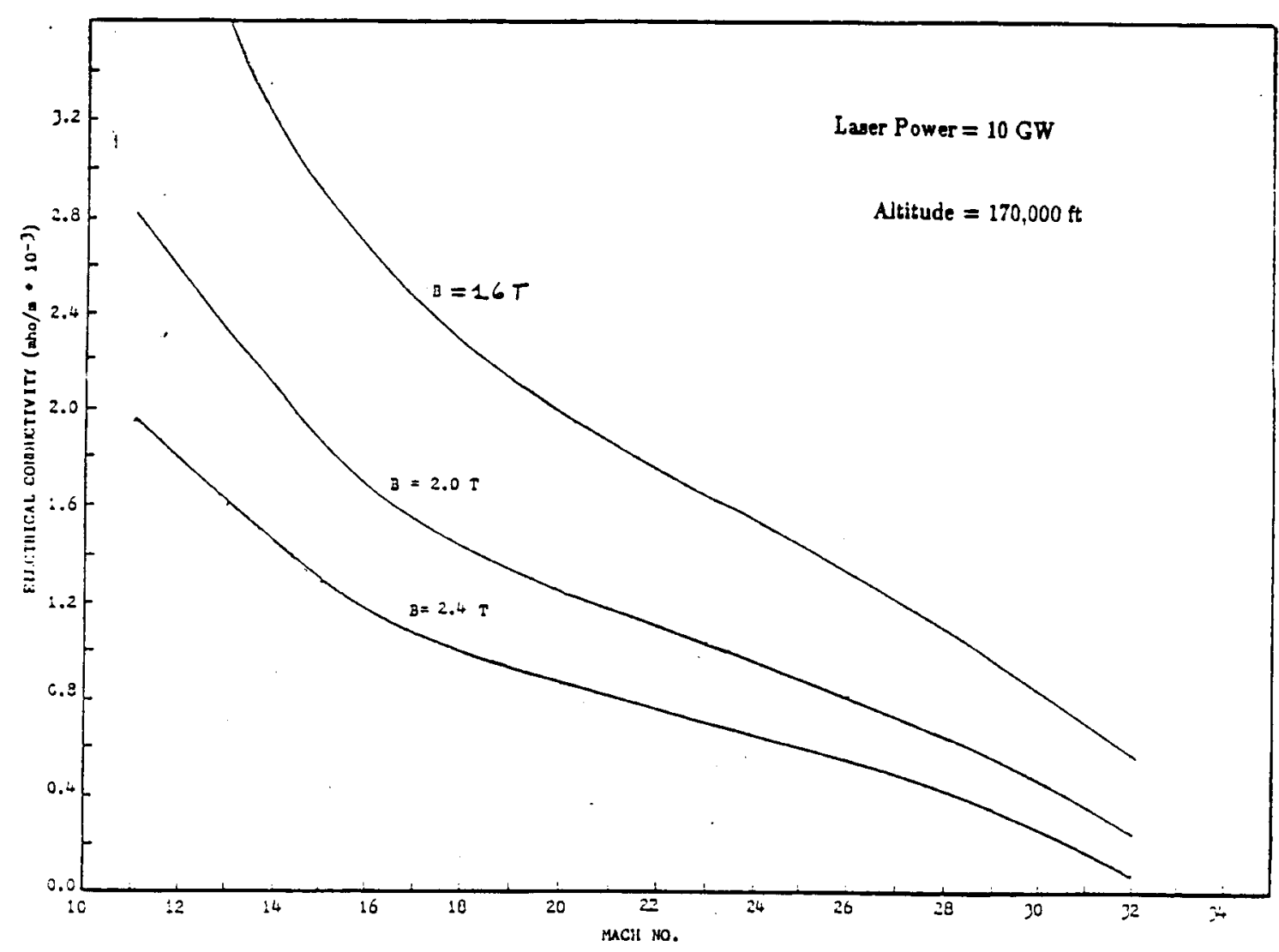

Figure 44. Conductivity enhancement required in the MHD accelerator. 


$$
\begin{gathered}
C=\frac{T}{P_{L}} \\
\dot{m}_{f}=(12 \dot{m}) T H R O T \\
T H R O T=\frac{P_{F} \text { used }}{P_{F} \text { full capacity }}
\end{gathered}
$$

where $\dot{m}_{f}$ is the total hydrogen mass flow rate for the MHD generators, $\tau_{g e n}$ is the thrust contribution from a single generator exhaust, and THROT is the throttle setting for the MHD generators. It is assumed for a given generator that the specific work, w(tot), and the conversion effieciency, $w($ tot $) / Q_{L}$, remain constant as the mass flow rate through the generator is decreased somewhat about its design value The electrical power required by the accelerator is found by integrating the electrical power input per unit volume over the volume of the accelerator:

$$
P_{F}=\iiint J_{y} E_{y} d(\mathrm{Vol})=B^{2} A_{F} \int_{0}^{L} \sigma u^{2} K(K-1) d x
$$

Curves for thrust, specific impulse, and coupling coefficient are shown in Fig. 43 over a range of altitudes and Mach numbers, for the case of $B=2$ Tesla and an available laser power of $10 \mathrm{GW}$ (the maximum power level for the Lightcraft). The curves are representitive of the typical behavior of the MHD-fanjet. A characteristic of MHD-fanjet mode is that fuel consumption (in this case, the hydrogen in the MHD generators) is constant for a given power level regardless of altitude or Mach number. As a result, the specific impulse and coupling coefficient are proportional to the thrust over the entire flight envelope, and it is possible to represent these three parameters with a single family of curves. The specific impulse of the MHD-fanjet is generally four to five times greater than that of the laser rocket $\left(I_{s p}=2000 \mathrm{~s}\right)$.

It is important to note that for the isothermal operation of the accelerator, the electrical conductivity (which is a strong function of temperature and a weaker function of pressure) is very nearly at the value corresponding to the static temperature at the inlet of the accelerator. Throughout most of the flight regime, the electrical conductivity corrresponding to the inlet temperature is not high enough for the MHD accelerator produce positive thrust. For this reason, it is necessary to enhance the conductivity through some additional means, such as microwave or radio frequency exitation, electron beams, or simple chemical seeding. The advantages and disadvantages of each of these alternatives is currently being investigated. In this model, the electrical conductivity, $\sigma_{T O T}$, required to absorb the power available from the MHD generators was computed by

$$
\sigma_{T O T}=\sigma+\sigma_{0}
$$

where $\sigma_{a}$ is a constant representing the amount by which the actual conductivity $\sigma$ must be increased such that $P_{F}$ (found by Eqn. (40), with $\sigma_{T O T}$ replacing $\sigma$ ) is equal to the available power produced 
by the MHD generators, $\dot{m}_{\rho} w\left(\right.$ tot); $\sigma_{s}$ is found iteratively. The maximum value of $\sigma_{s}$, at a given Mach number and altitude, occurs at the maximum laser power of $10 \mathrm{GW} . \sigma_{8}$ verses fight Mach number at various magnetic field values is plotted in Fig. 44 for a laser power of 10GW at an altitude of $170,000 \mathrm{ft}$. 


\section{CHAPTER V}

\section{ROCKET MODE}

At altitudes greater than $200,000 \mathrm{ft}$, the MHD-fanjet begins to function essentially as a rocket since the thrust from the MHD generator hydrogen exhaust begins constitute a large fraction of the total MHD-fanjet thrust, due to the decreasing ambient density. Consequently, at an altitude of 260,000ft, the applied magnetic fields for the MHD generators and accelerator are shut off, and the Lightcraft transitions into a pure rocket mode - the twelve MHD generators become laser-heated hydrogen rockets. The laser absorption chamber and MHD generator duct form a convergingdiverging nozzle in which the throat area is the inlet of the MHD generator. In the trajectories currently used, the accelerators and generators are shut off at 260,000ft. From here, the Lightcraft coasts out to about $600,000 \mathrm{ft}$, at which point, a rocket burn is used for orbital insertion. However, in future studies, the use of an additional rocket burn at the end of the MHD-fanjet mode may be investigated as a possible strategy for minimizing total energy consumption.

\section{A. ROCKET PERFORMANCE}

For a given MHD generator (ie, the HIGH or LOW case generator), the geometry of the generator duct and laser absorption chamber are fixed. However, since the laser absorption chamber temperature and pressure can be varied about their MHD-fanjet values (by altering the laser energy into the chamber and the pumping pressure of the hydrogen propellant), and the absorption chamber/generator duct constitutes a converging-diverging nozzle, the MHD generators can be used as rocket nozzles, assuming the back pressure (ambient pressure) is low enough to allow supersonic flow at the exit of the generator duct. As noted, the minimum altitude at which the rocket mode will be used is $260,000 \mathrm{ft}$, which corresponds to a maximum back pressure of $1.74 \times 10^{-5}$ atm; thus, for entrance stagnation pressures on the order of 1 atm (or more), the flow will be supersonic and underexpanded at the generator exit, with further expansion occuring over the plug nozzle.

A detailed analysis of the hydrogen flow through the generator duct in the rocket mode was not performed, although such an analysis will soon be carried out using the model developed for the MHD generator in the MHD-fanjet mode, with the electromagnetic terms removed and the constant velocity condition replaced by a prescribed area distribution for the generator duct. In order to carry out the trajectory analysis for the rocket mode, some engineering assumptions were made. The thermal efficiency of the generator duct operating as a rocket was assumed to be $66 \%$ (reasonable for the high operating temperatures) and the thrust from each rocket was defined as the 
product of the hydrogen mass flow rate times the exit velocity (the expansion over the plug nozzle was ignored). Defining $I_{o p}$ as the specific impulse and $\mathrm{C}$ as the coupling coefficient, the thermal efficiency, $\eta_{t h}$, can be written as $\eta_{t h}=\frac{1}{2} g I_{m p} C$, where $g$ is the gravitational acceleration. We can generate very high speciflc impulses for the rocket since high temperatures $(15,000$ to $20,000 \mathrm{~K})$ are easily obtained using standing LSC waves in the absorption chamber, however, in order to also obtain a reasonable coupling coefflcient of $67 \mathrm{~N} / \mathrm{MW}$, a speciflc impulse of $2000 \mathrm{sec}$ was decided on as the design point for the laser-heated rockets.

The desired specific impulse can be generated for a given generator duct by providing the proper stagnation temperature and pressure at the absorption chamber inlet. Each rocket must also produce a minimum thrust of approximately $18.6 \mathrm{kN}$ to satisfy certain requirements for the optimization of the trajectory (assuming all 12 rockets are operating at the same capacity). The mass flow rate for each rocket must therefore be greater than or equal to $0.948 \mathrm{~kg} / \mathrm{s}$ for a specific impulse of $2000 \mathrm{sec}$. For a given TO (Stagnation temperature) and PO (Stagnation pressure), the mass flow rate per unit area necessary for a choked flow is determined, and for a given generator inlet area, $A_{i}=D_{i} D_{y}$ (ie, the throat area of the rocket nozzle), the mass flow rate is determined. Using rocket performance data for high temperature hydrogen generated by Patch [?], it was possible to check the feasibility of achieving a specific impulse of $2000 \mathrm{sec}$ and a minumum thrust of 18.6 $\mathrm{kN}$ for the HIGH and LOW case generators. For the HIGH generator, the exit to throat area ratio is 42. For TO $=8000 \mathrm{~K}$ and $\mathrm{PO}=60$ atm, linear interpolation of Patch's data yields: $I_{\text {ap }}=20408$, an exit pressure $P e$ of $.33 \mathrm{~atm}$, and a mass flow rate $\dot{m}$ of $0.898 \mathrm{~kg} / \mathrm{s}$. Using the same conditions for the LOW generator (which has a exit to throat area ratio of 20.4) yields : $I_{\text {op }}=1955 \mathrm{~s}, \mathrm{Pe}=$ $0.52 \mathrm{~atm}$, and $\dot{m}=4.09 \mathrm{~kg} / \mathrm{s}$. Of course, these calculations do not account for the effect of the wall shape between the throat and exit of the nozzle, nor the heat. and friction looses at the walls. They do indicate, however, that the numbers used for the specific impulse and coupling coefficient in the trajectory analysis for the rocket mode are reasonable. 


\section{CHAPTER VI TRAJECTORY ANALYSIS}

The trajectory analysis of any launch vehicle becomes a critical step in the overall system integration process. Many important engine/vehicle related characteristics of the craft must come together for the final product - performance. Launch vehicle performance is typically measured in terms of payload capability, which for the Apollo Lightcraft is the five person crew (plus space suits) which totals 500 kilograms. This vehicle must be capable of attaining low Earth orbit with the available amount of propellant while minimizing the total laser energy consumed along the insertion trajectory.

The trajectory was evaluated using a computer tool called SORT (Simulation and Optimization of Rocket Trajectories) which was written by McDonnell Douglas Astronautics Co. for NASA to design space shuttle trajectories [2]. The code is suffliently general such that it can analyze any trajectory (even those for other planets), and model all important environmental parameters that affect the vehicle dynamics. Engine performance, vehicle aerodynamics, guidance algorithms, and mass histories interact with with atmosphere and gravity models.

The capability of the SORT program is quite extensive in its use of these sophisticated vehicle and environmental models. The program can iterate on trajectory parameters to optimize performance, achieve a desired criteria, or constrain the solution to avoid some specified limit. These capabilities make SORT one of the most powerful trajectory analysis tools available in the world.

Even with all of the generality built into SORT certain modifications were required for the Apollo Lightcraft vehicle. The most signiflcant software modiflcation involved the unique energy source, a laser. A new vehicle steering option was encoded so that the Lightcraft could always point at the laser power relay satellite which initially passes overhead (See Fig. 45). Since the use of laser propulsion was not anticipated by the authors of SORT, software changes were also needed in the engine model in order to evaluate the total amount of laser energy expended along the trajectory. After these modifications to the code were included, SORT was capable of modeling Lightcraft performance to a high degree of accuracy.

\section{A. VHHICLE AND ENVIRONMINTAL MODGLS}

\section{Aerodynamics}

For this preliminary performance analysis vehicle drag was the only aerodynamic force (or moment) that was modeled. Since the Lightcraft is axisymmetric, it produces no lift at zero angle- 

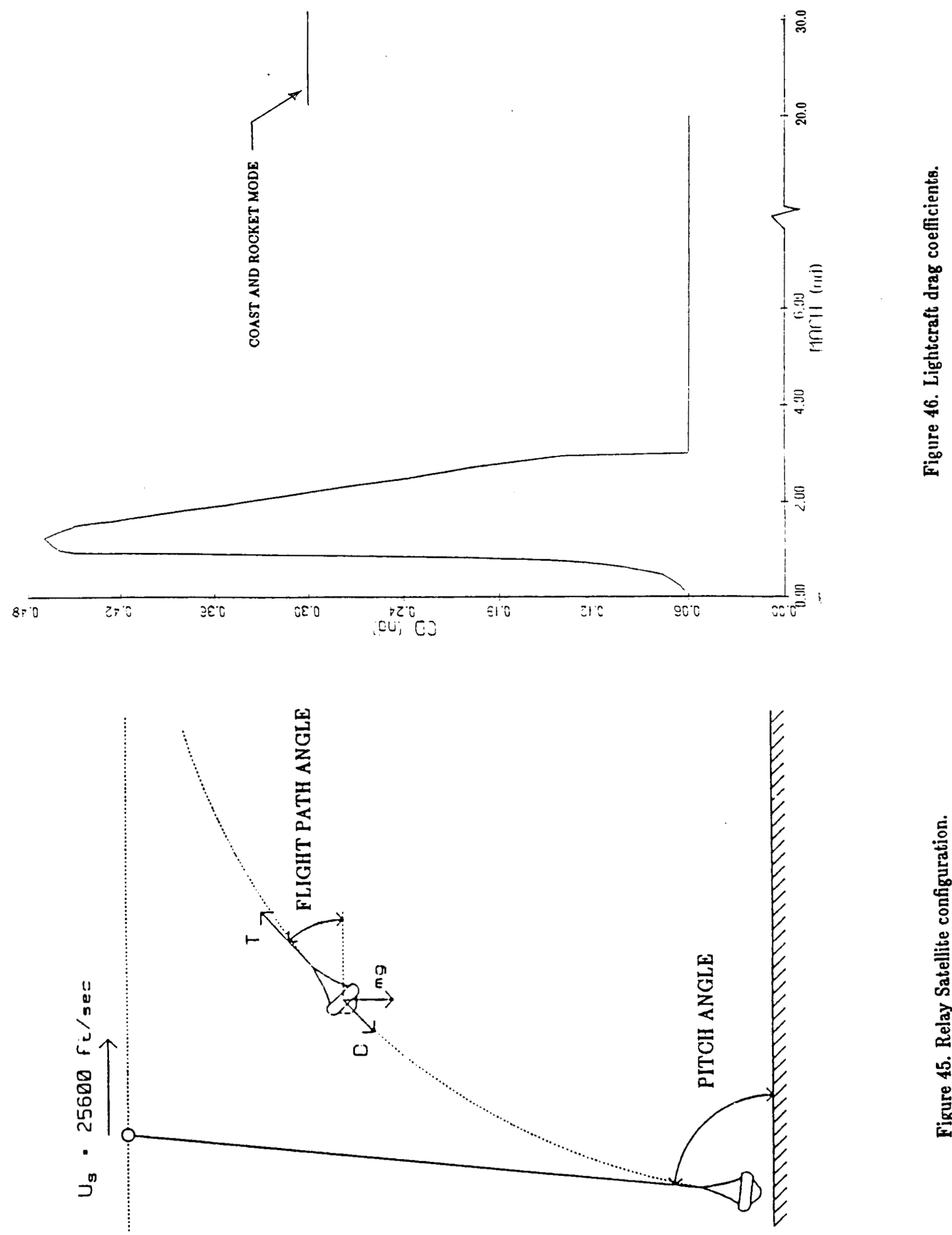

 
of-attack. During flight the vehicle angle-of-attack stays fairly small due to the restrictive steering requirements and high thrust to weight ratios. It is suspected that aerodynamic lift can possibly be used to beneflt Lightcraft performance along the trajectory.

The vehicle drag profile is shown in Fig. 46 The very low drag coefficients indicated for most Mach numbers is a result of the vehicle configuration, which effectively is a flying engine. In the analysis of hypersonic air breathing launch vehicles, it is not always immediately evident as to what should be classified as "engine " versus "airframe" - especially when large portions of the vehicle forebody provide the hypersonic inlet function. Hence careful inventory must be kept to avoid penalizing the engine for what might be more appropriately accounted against the the vehicle airframe.

For example, the large form drag normally associated with "blunt " supersonic bodies is treated as a loss term in in the engine model. Almost all of the Lightcraft forebody is the supersonic inlet and the pressure on this surface (as well as the external nozzle) are modeled in the engine calculations. The shroud is the only remaining aerodynamic interaction with the freestream. The drag produced by this surface will vary considerably depending on the deflection or curvature of the shroud. The drag coefficient values past Mach 3, shown in Fig. 46, correspond to 15 degree deflections of the leading and trailing edges (see Fig. 47). The large drag increase from Mach .9 to Mach 3.0 is an artifact of "spillage" drag. The inlet does not capture all of the of the shocked air until the vehicle reaches Mach 3, the design Mach number of the Apollo Lightcraft inlet. Hence, below Mach 3, the air which is slowed by the shock, but not ingested by the engine is attributed to vehicular drag. The shroud is designed to translate fore and aft, such that all of the shocked air is swallowed at flight speeds beyond Mach 3.

\section{Engine}

Detailed discussions for each engine model are presented in earlier sections of this report. The flight performance for each engine was calculated for a wide range of altitudes, Mach numbers, and power settings. These values were then loaded into trivariant tables accessible to the program. The SORT program interpolates this data to find the thrust, specific impulse and power consumption at any point along the trajectory. The engine model also calculates the cumulative laser energy used throughout the launch trajectory, which also includes atmospheric attenuation of the laser beam.

\section{Steering}

The Apollo Lightcraft must point at the laser relay satellite at all times during powered flight. Two laser power source locations were asgumed for most of the analyses which have been carried out to date. One location is directly overhead in a very high orbit, where no significant movement occurs with respect to the earth's surface during the boost; the other location considers 


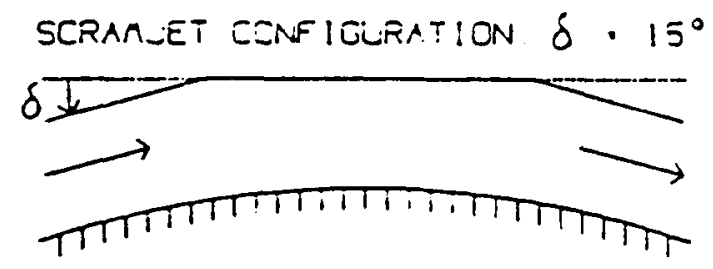

Figure 47. Lightcraft cowl profile.

MASS BREAKDOWN FOR "APOLLO LIGHTCRAFT"

\begin{tabular}{lcc}
\hline \multicolumn{1}{c}{ COMPONENT } & MASS (KG) & WEIGHT (LBS) \\
\hline & & \\
PAYLOAD (5 PERSON CREW) & 500. & 1100. \\
STRUCTURE (+ PRIMARY OPTICS) & 2550. & 5620. \\
THEFMAL PROTECTION SYSTEM & 450. & 990. \\
SHROUD (+ SECONDARY OPTICS) & 180. & 400. \\
SHROUD NOZZLE (VARIABLE AREA) & 180. & 400. \\
LASER-HEATED ROCKETS & 400. & 880. \\
MID GENERATORS & 200. & 440. \\
MHD AIR ACCELERATOR & 200. & 440. \\
LANDING GEAR (RETRACTABLE) & 170. & 380. \\
6-AXIS CONTROL ROCKETS & 100. & 220. \\
CHUTE AND FLOTATION & 90. & 200. \\
AVIONICS, ENVIRON. CONTROL, & 120. & 260. \\
AND PERSONAL PROVISION & 80. & 180. \\
I.HZ TANK (INSULATED) & 330. & 740. \\
LHZ PROPELLANT & & $12,250$. LBS \\
\hline & $5550 . \mathrm{KG}$ & \\
\hline & &
\end{tabular}

Figure 48. Mass Breakdown for the Apollo Lighteraft. 
a second relay satellite passing overhead in a low orbit (100-200 NM). Nearly all of the acceleration takes place as the vehicle chases the low altitude relay satellite. Currently, the steering model works only for satellites passing directly over the launch site, and further improvements should permit the inclusion of relay satellites with a more general ground track.

\section{Mass Properties}

The preliminary weight breakdown for the Apollo Lightcraft can be found in Fig. 48. The program can model variations in the center-of-gravity (CG) location, but the present analysis placed the vehicle CG on the centerline (along with the engine) to simplify the thrust-pointing requirements.

\section{Gravity}

A complete Earth gravity model exists in the SORT code which includes J2, J3, and $\mathrm{J4}$ effects. The program continuously calculates the orbital parameters throughout the boost trajectory.

\section{Atmosphere}

The atmospheric data loaded into SORT was the ARDC Model Atmosphere [6]. Although wind can be a significant aerodynamic perturbation none was included at this stage of the analysis.

\section{B. TRAJECTORY SIMULATION}

The SORT program treats the trajectory as a specified sequence of maneuvers or phases. Each event is defined by the user at appropriate places in the simulation so that new inputs can be assembled into the trajectory. The launch vehicle flies from event to event until, finally, it obtains the orbital parameters necessary to achieve the desired orbit altitude. The craft then coasts until it reaches apogee, and performs a final orbit-insertion burn. The SORT program has iteration capability that can be used to optimize total propellant weight (or laser energy) used during ascent. A typical trajectory sequence is described below.

\section{Liftoff in ERH thruster mode}

The vehicle climbs vertically (using the high orbit satellite) to a specified altitude. This "pop-up" maneuver lifts the vehicle out of the dense atmosphere before attempting the acceleration run. Thereby, the high drag losses and structural loads associated with very large dynamic pressures are avoided. Simulations prove that the pop-up maneuver is more advantageous than accelerating "off the deck". Since the ERH thruster mode is completely air-breathing, the manuever does not consume any $\mathrm{LH}_{2}$ propellant and only a small penalty in laser energy cost is incurred - which is paid back later in the trajectory, because the aerodynamic forces (and hence, required laser energy) are reduced. 


\section{Pitchover (ERH thruster)}

At the specifled (or optimized) altitude the vehicle pitches over to begin receiving power from the low altitude relay satellite. The exact zenith angle at which to engage the relay satellite is determined iteratively to achieve maximum performance. If the satellite is engaged when it is too high overhead, the vehicle will leave the atmosphere before high velocities are reached, which necessitates more use of the rocket engine. If engaged too late (i.e., when the satellite is too far from vertical the satellite will disappear over the horizon before orbital speeds are attained; or the trajectory will not climb high enough to prevent increased aerodynamic drag which is associated with additional expenditures of fuel or laser energy.

This maneuver effectively initiates the acceleration portion of the flight. As the vehicle pitches over, the pulse repetition frequency of the ERH engine is increased to accelerate the craft through the transonic region towards Mach 3. As the ram drag increases net effective thrust of the ERH thruster decays.

\section{Scramjet Mode}

To increase vehicle thrust the next air-breathing engine mode begins and the ERH thruster is turned off. Currently the scramjet mode is used to accelerate the vehicle from about Mach 3 to 11. The scramjet portion is flown using a fixed (or optimized) maximum laser power level. At moderate altitudes, the scramjet can handle relatively high power inputs because of large mass flow rates and "low" inlet temperatures. However all of the $10 \mathrm{GW}$ (max laser power limit) is not needed. Three Gigawatts of laser power can accelerate the vehicle at up to $6 \mathrm{~g}$ 's (even with vehicle drag forces included). If the craft accelerates too quickly, the aerodynamic forces build up, and as a result acceleration then declines while structural loads increase. At high Mach numbers, or as the atmosphere thins out at higher altitudes the engine can only add small amounts of energy to the air flow without exceeding the temperature limits of the engine (set at $10000^{\circ} R$ ). As thrust decays near Mach 11 and vehicle acceleration approaches "break even " $1 \mathrm{G}$ ), the MHD fanjet will start.

\section{MHD Fanjet Mode}

When the scramjet fades out and acceleration drops below $40 \mathrm{ft} / \mathrm{sec}^{2}$ the fanjet is started. By waiting as long as possible to switch to fanjet it is possible to conserve onboard $\mathrm{LH}_{2}$ propellant. The fanjet mode is used to accelerate the vehicle right up to orbital insertion speeds. As long as it captures significant mass flow rates of air to accelerate, the MHD fanjet has specific impulses far above the rocket mode. Hence, the rocket is used only for final "burns " in orbit.

The final velocity desired at fanjet termination is a function of altitude, flight path angle and the drag experienced as the vehicle coasts up to the desired altitude (e.g. $100 \mathrm{NM}$ ) to make the final orbit circularization burn. There is no easy analytical solution to this targeting problem so the 
program iterates to find the fanjet cutoff velocity.

\section{Coast to Apogee}

This is the engineless phase where the vehicle coasts up to apogee. After the fanjet cuts off the drag coefflcient is increased to .3 since the forebody drag increases significantly when the shocked air is not re-accelerated within the cowl.

\section{Final Insertion}

The rocket is started as the vehicle reaches apogee. This burn is terminated as the inertial velocity reaches the necessary value for circular orbit at that altitude.

Many trajectories are run in any optimizing attempt. Typically the performance indicator propellant weight was optimized, although total laser energy was run also. Analysis in the future will focus on the minimization of laser energy. The key parameters which were varied in the attempt to optimize weight were the initial position of the relay satellite, power available to the engines (throttle setting), and altitude for the pitchover maneuver. Trajectory optimization is a never- ending task (e.g. engineers are still tweaking Space Shuttle trajectories to discover more payload capability), but the results so far give a baseline indication of the Apollo Lightcraft performance potential.

\section{RJSULTS}

The trajectory analyses to date using SORT have just barely scratched the surface of the comprehensive performance optimization problem. However these simulations do give highly accurate answers for the selected models and input data. Assuming the vehicle models (engine, drag, etc.) are fairly accurate, the resultant performance reported here would certainly be attainable. Although not optimal, the current results indicate the types of problems to be encountered, and present conservative performance capabilities (within the accuracies of the models and assumptions). Further, more detailed analyses can be expected to improve upon these results.

The earliest trajectory concepts for the Apollo Lightcraft focussed on maximum thrust takeoffs, reasoning that high accelerations would give the best performance. That approach does not bear fruit for this advanced launch vehicle which has incredible thrust-to-weight capability. The "max-G" type of launch quickly builds up very high aerodynamic loads (dynamic pressures $i 10000$ psf), and the drag rises to meet the thrust. Engine failure at these dynamic pressures could result in decellerations as large as $50 \mathrm{G}$ 's or morel Alternatively, low acceleration takeoffs had problems with the satellite disappearing over the horizon before the insertion was complete.

As the SORT program became available a much improved analysis of the trajectory began. It became obvious that the problem of the high drag transonic region could be eased by flying with 
lower dynamic pressures. This eventually led to the origination of the pop-up maneuver concept, wherein the vehicle climbs out subsonically to a high altitude before attempting to accelerate through this drag barrier.

When using a single relay satellite for a slow climb-out the vehicle would gradually pitch over to follow the satellite. As this occurrs, more thrust would be needed to maintain at least one (1) $G$ in the vertical direction. To solve this problem, it was suggested that a very high altitude satellite (e.g. geosynchronous) would move slowly (or not at all), allowing the vehicle to climb almost vertically. This option also required very low laser power requirements, which opened the possibility of using microwave power for the vertical climb. Since microwaves are not significantly attenuated by clouds, this would allow all-weather operation. Even on a cloudy day one could fly to orbit in a lightcraft, because the microwave power would boost the vehicle to 30000 to 50000 feet (above the cloud tops) - before a separate high power laser at another location was engaged.

Fig. 49 illustrates the principle features of such a trajectory. The plot shows the pop-up maneuver with a moderate acceleration at liftoff which soon levels out to a constant $1 \mathrm{G}$ as the vehicle climbs through the lower atmosphere. As the Lightcraft reaches 35000 feet (60 sec), it pitches over and the ERH thruster is throttled up. Near Mach $3(90 \mathrm{sec})$ the ERH engine thrust drops rapidly and then the scramjet starts. Once again there is a large acceleration spike followed by a dip which is caused by drag build up. (The jumpy nature of the acceleration is in part due to this thrust/drag interaction, as well as some coarseness in the engine input data.) The sramjet "dies " near Mach $11(160 \mathrm{sec})$, and the MHD fanjet is started. Acceleration during the fanjet cycle decreases as the coupling coefflcient declines with increasing Mach number. It tails up near the end of its phase because the coupling coefflcient levels out some (the generator exhaust is now providing a significant portion of the thrust), as the drag continues to decrease. Insertion is completed near 270 seconds and then the vehicle coasts up to the apogee $(1000 \mathrm{sec})$ where a final rocket burn circularizes the orbit.

The disadvantage of this latter idea is the requirement for two satellites and, of course, the transition between them. The high altitude satellite increases the complexity of orbital mechanics necessary to open a "launch window". A traditional geostationary satellite with an orbital inclination of zero degrees would appear quite a bit below maximum azimuth in all of the United States, or any location at significantly distances from the equator. The global problems of satellite power system architecture and relay satellite availability has yet to be studied in any detail.

The most recent analysis is exploring the potential for combining the basic two-satellite boost system into a single into a single relay satellite, for fair weather launches. Fig. 50 compares the baseline trajectory which has a pop-up maneuver to $35000 \mathrm{ft}$. to an off-the-deck flight with a 

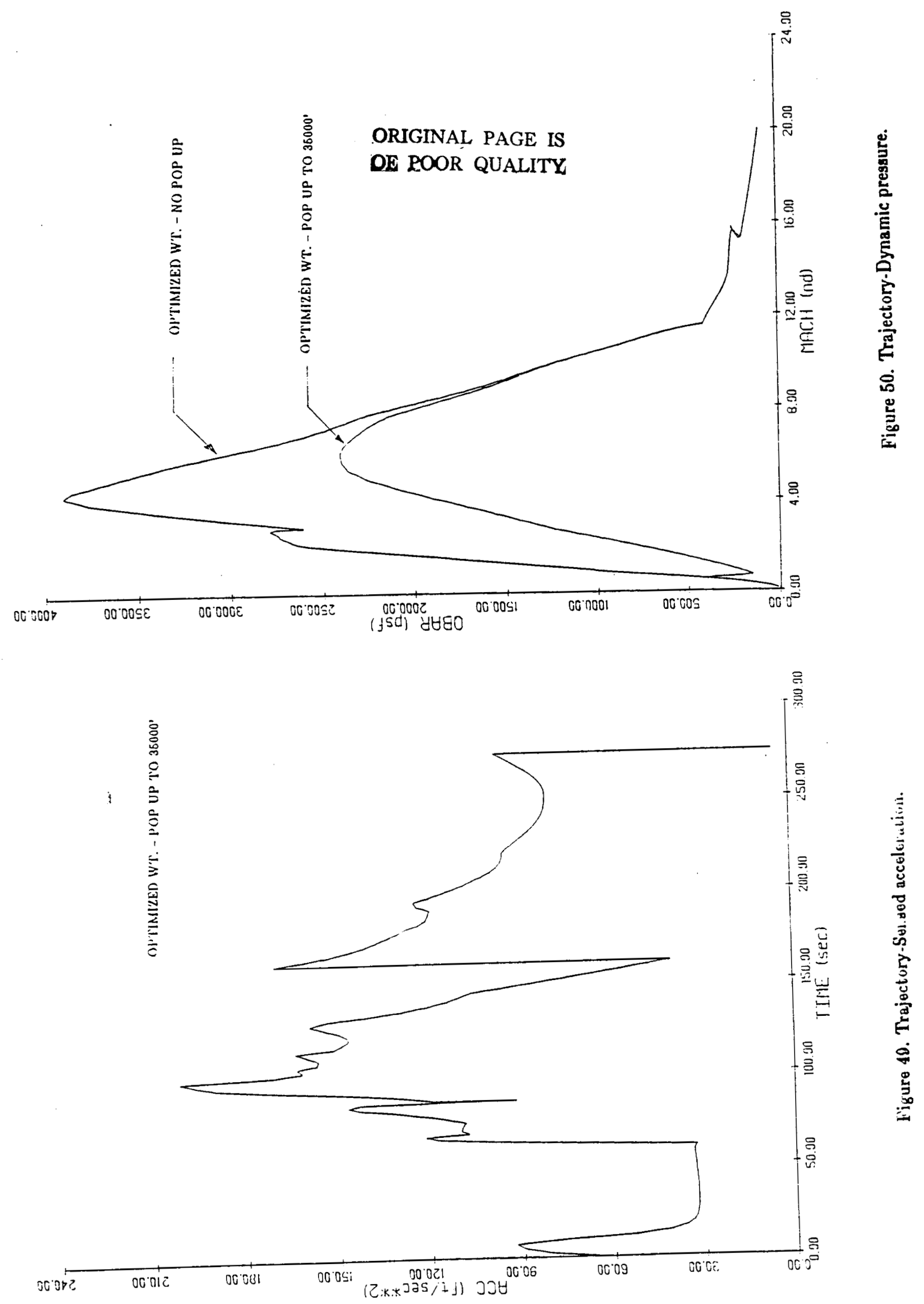
linearly increasing throttle setting to counter the slow pitch-over as it climbs. The dynamic pressure is noticeably higher in the latter case because speeds are higher at any given altitude. The total energy required was also about $70 \mathrm{GW}$-sec, higher due primarily to the larger drag forces on the vehicle. Fig. 51 shows that the primary difference in energy cost is created from about Mach 1 to Mach 4 where the higher dynamic pressure is combined with the large drag coefficient in that region. After Mach 5 the curves are effectively parallel to the baseline trajectory. Another potential problem with off-the-deck fights is that the ERH thruster cannot reach Mach 3 until about 45000 feet, because the ram drag is otherwise too large at these speeds and altitudes.The notch in the dynamic pressure (Fig. 50) is evidence that the ERH thruster is struggling to get to Mach 3 . As the acceleration from the thruster decays the dynamic pressure actually begins to decrease before the scramjet kicks in.

Much of the effort to date has been spent trying to minimize the propellant needed to reach final orbit and the results were surprising. Changing parameters that affect primarily the first part of the trajectory (i.e. pop-up altitude, ERH performance, etc.) has little effect on the final value of propellant weight. Apparently the "iterator " finds a solution so that the MHD fanjet portion of the flight is very similar, even though the early part of the trajectory isn't. Changing performance characteristics of the fanjet or the maximum available laser power levels obviously affects the solution, but the general indications are that the fanjet concept can work very well in this type of vehicle.

The MHD fanjet analysis section described two different generators with different assumptions and performance specifications. Even when the MHD generator performance is reduced to the lower, more conservative generator, the results are not discouraging. The performance difference between the two generators gives a specific impulse reduction of a factor 1.8; e.g., if the performance with the optimistic generator was 9000 then the pessimistic performance is $5000 \mathrm{sec}$. This magnitude of specific impulse loss would be disasterous for a chemical engine with an $I_{\mathrm{op}} \approx 400 \mathrm{sec}$ and a propellant fraction of $85 \%$.

Since the propellant fraction for the Apollo lightcraft is so low (about 5-10\%), the additional fuel required remains fairly linear with the specific impulse change. The propellant required using the optimistic MHD generator is about $650 \mathrm{lbm}$ and $1100 \mathrm{lbm}$ for the more conservative case. This would keep the propellant fraction under 10 percent of the gross lift-off weight. The biggest problem would not be weight, but volume since liquid hydrogen has a very low density. Nevertheless, with such low propellant fractions the performance of this vehicle is clearly revolutionary. It is interesting that with propellant fractions of about $15 \%$, escape velocities could be attained using the MHD fanjet cycle. 
The fanjet, however, is not without drawbacks. The coupling coefficient of the fanjet decreases with increasing flight Mach number, as do all air-breathing engines. At around Mach 20 (depending on the altitude), the coupling coefflient drops below that of the rocket; therefore, propellant weight is minimized at the expense of increased laser energy expenditure. How these two engine modes are to be traded against each other will depend largely on the cost of each quantity (i.e., $\mathrm{LH}_{2}$ vs. laser energy), as well as the effects of changing vehicle configuration.

A computer run was made to minimize the total laser energy while making very few changes to the input parameters.as a Fig. 51 shows the energy cost for these two cases (as well as the "offthe-deck " flight). The curves are almost coincident until Mach 5 where a slight difference in flight path angle is "lofting " the trajectory above the baseline case - reducing drag and hence energy. (The switch-backs near Mach 15 are caused by an atmospheric inflection point where the pressure and density change abruptly; this causes the speed of sound calculation to indicate increasing values for a short period. There is an apparent error in the source for the atmospheric data at $170000 \mathrm{ft}$ which causes this anomaly. The vehicle is not slowing down, but the speed of sound increase causes the Mach number to drop for a short period.) Near the end of all the lines there is a horizontal line. This is the coast period where no energy is added. The final section shows the energy addition for the rocket mode. The optimized energy case clearly uses more energy in the rocket mode. Since the coupling coefficient is higher for the rocket than the fanjet at these speeds, the optimization algorithm selected a case that uses more rocket thrust than the weight optimized case. However, the optimized energy case uses $150 \mathrm{lbm}$ more propellant than the optimized weight.

The iterator was trying to reduce the fanjet phase (and the drag), and the only method available to it (in this run) was to loft the trajectory. This causes the fanjet to terminate earlier because at higher flight path angles orbital mechanics dictates that the velocity be lower - to reach a fixed apogee altitude. This lofting is evident on Figs. 52 and 53. The primary input difference between the two cases is that the relay satellite is about 3 degrees higher from the horizon at pitchover for the lofted trajectory. Fig. 53 clearly shows the pitchover maneuver at about 60 seconds, and the difference in flight path angles at thrust termination.

\section{SUMMARY}

The trajectory analysis will need to continue as the engine and aerodynamic models evolve and the final results may end up considerably different than those presented here. This does not imply that the current analysis is crude or inaccurate, because it is quite precise within the confines of the models and inputs. The predicted performance for the Apollo Lightcraft is undoubtedly revolutionary, and further analysis will continue to define this new frontier. 


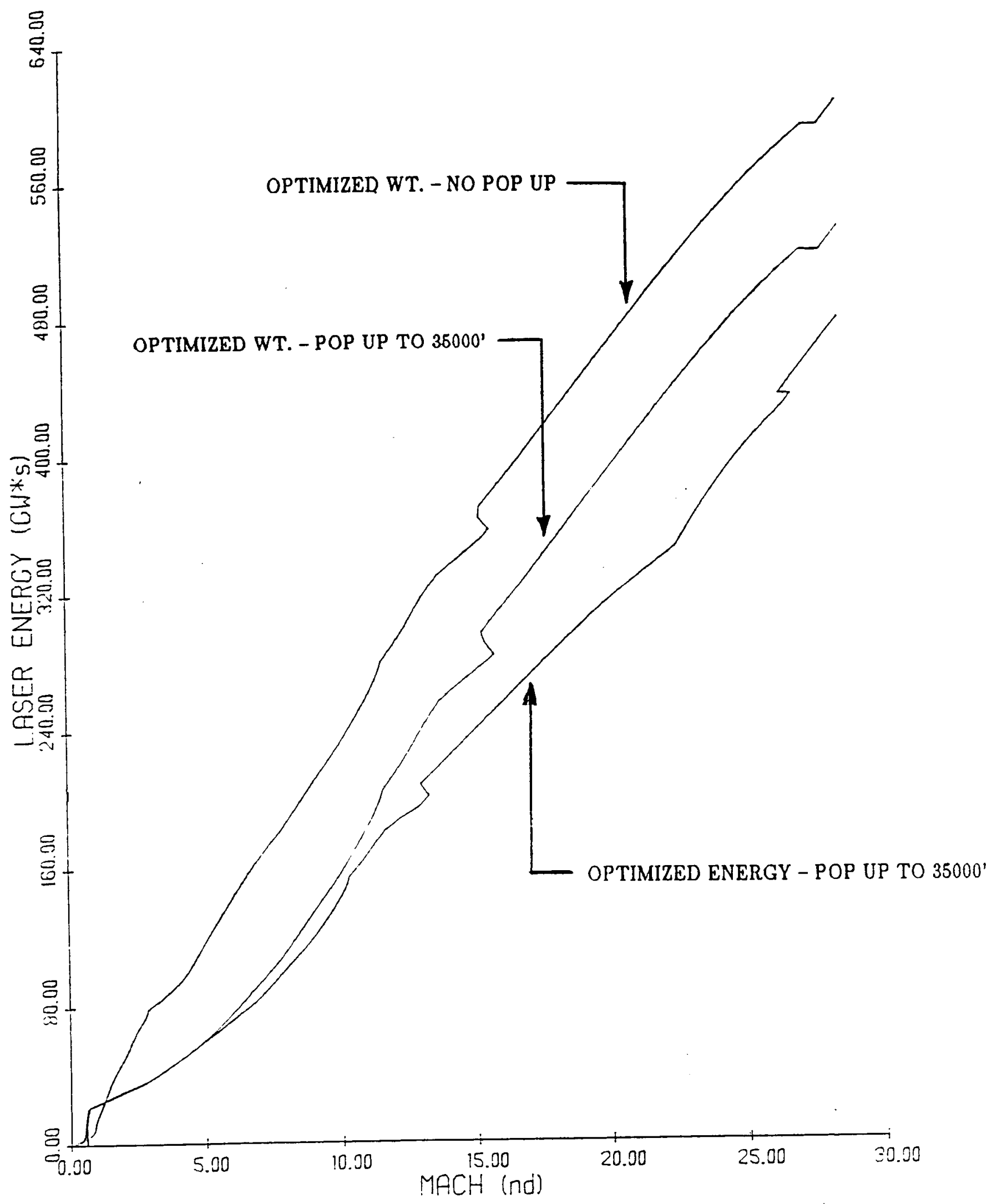

Figure 51. Trajectory-Total Laser Energy. 


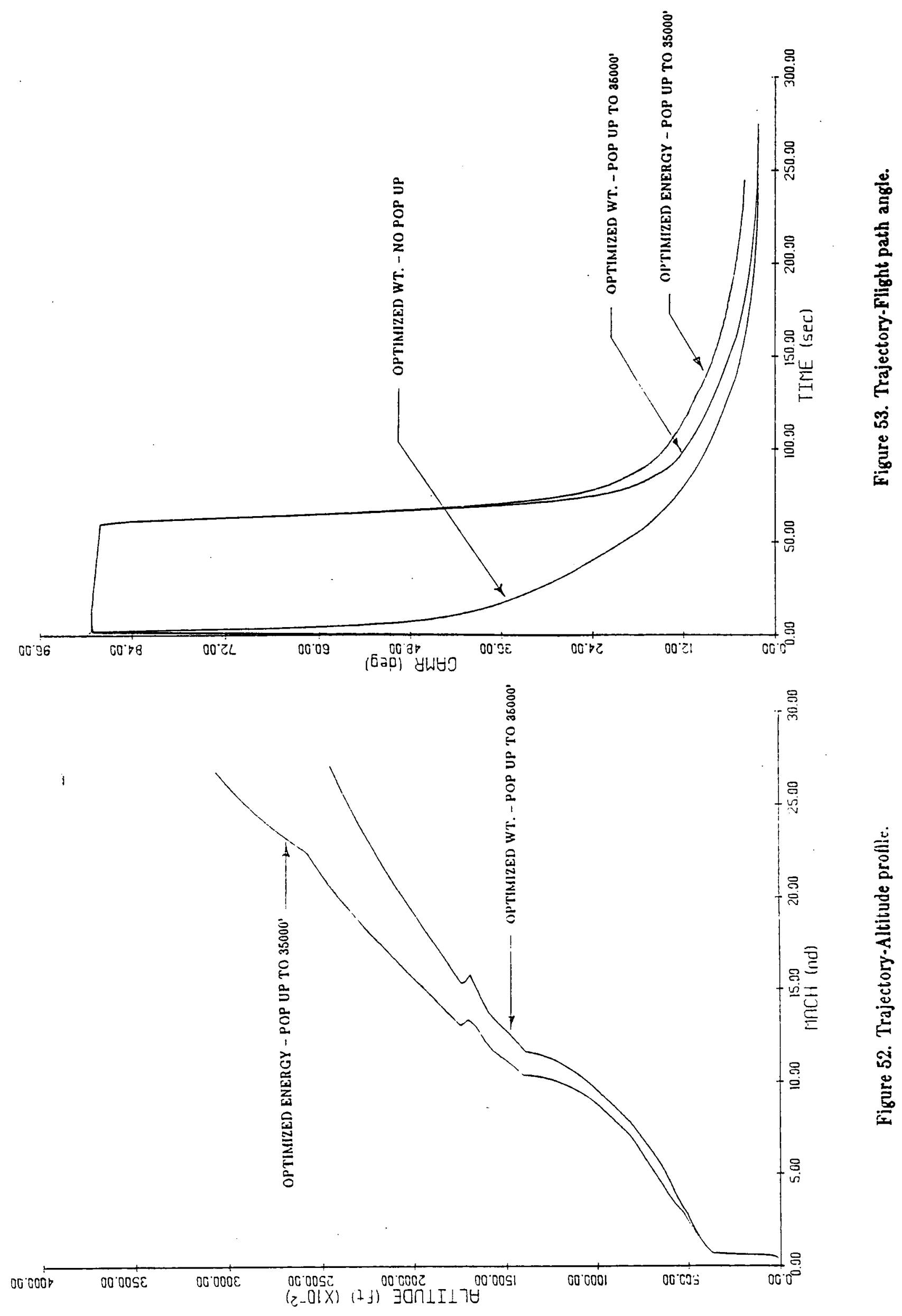


Currently there are a few tender areas in the trajectory modelling effort. Among them is the possibility that more accurate models of the ERH and scramjet engines could leave a "hole" in the thrust spectrum around Mach 3. If this was to occur another engine would be necessary (probably a ramjet) which would require configuration changes for the shroud and annular engine duct. Similarly, the MHD fanjet model does not presently have an analysis of conductivity enhancement necessary for significant electromagnetic power absorption. Implementation of such a model might indicate diffculty generating thrust at "low" Mach numbers (i.e., near scramjet fade-out). Aerodynamic heating and engine heat transfer has largely been ignored and this may drive the vehicle into more benign fight conditions. Viscous effects (boundary layers, etc.) have been neglected and this may significantly alter engine performance and vehicle drag coefficients.

Even with the above-mentioned concerns, the performance of the Apollo Lightcraft goes far beyond that available from any launch vehicle existing today. These early models of revolutionary engines are stepping stones to more powerful launch vehicles. The present analysis demonstrates the incredible potential of beamed-energy propulsion when united with innovative launch vehicle concepts. 


\section{CHAPTER VII}

\section{SUMMARY}

The central objective of this NASA/USRA-supported effort was to investigate the performance of a revolutionary combined-cycle engine suitable for the Apollo Lightcraft. The mission for this advanced SSTO shuttlecraft is to transport a five person crew to low Earth orbit in three minutes, or anywhere on the globe in one half hour. Beam power for propulsion was limited to 10 billion watts, a typical capacity quoted in studies of future satellite solar power stations.

The combined-cycle engine seems to be feasible, and has now withstood a first critical inquiry into the propulsive physics. The group has not identifled any performance projections that violate basic physical principles. This first-order performance analysis has unearthed a number of necessary refinements for each of the engine mode models, which are addressed below.

\section{A. ERH THRUSTER MODEL}

The operation of this innovative pulsejet engine can liken to a traditional Otto cycle complete with intake, compression, ignition (i.e combustion), and exhaust functions. A more complete understanding of the exhaust/intake phenomena (heretofore called "refresh ") is required to ascertain its effect upon the maximum time-averaged thrust capacity of the ERH Thruster. Also, the present assumptions on the peak (LSD-wave-heated) "combustion "pressure are being re-examined, in light of new evidence which suggest somewhat lower values.

\section{B. RAMJET/SCRAMJET MODEL}

Although the ramjet mode is presently "on the back burner ", it still may be resurrected if a 'hole'(in propulsive thrust) appears between Mach 2.5 and 3.5. Otherwise, all attention is focused on scramjet engine model refinements. Since scramjet temperatures can easily exceed $10,000 \mathrm{R}$ at high flight Mach numbers, efforts are underway to include an accurate "real gas "code. The group has a current copy of the NASA-Lewis "McBride "code, and plans to exercise it over the summer. Further model improvements are necessary in algorithms used for inlet and nozzle functions, especially with regard to frozen-flow effects.

\section{MHD-FANJET MODEL}


The MHD-fanjet engine is probably the most critical "link " in the entire combined-cycle engine. The second most critical is the rocket mode. Unlike the ERH thruster and scramjet, they both have flnite fuel specific impulse and dictate the total fuel load required to meet a specific Earthto-Orbit mission. The ratio (by weight) of fuel consumed is roughly $80 \% / 20 \%$ by the MHD-fanjet and rocket, respectively.

As mentioned earlier, the MHD-Fanjet engine is comprised of two major components: the laser-heated MHD generators, and the annular MHD air accelerator. The most urgent improvement required in the MHD generator code is the inclusion of a credible radiative heat transfer model. Hydrogen gas temperatures may reach 15,000 to $20,000 \mathrm{~K}$, and radiation effects cannot be ignored under these conditions.

For the MHD accelerator code, a more through understanding of the air conductivity requirements throughout the Mach 11-30 flight environment, is in order. Although several viable candidates exist for enhancing the normal air conductivity, the associated mass loss (ie., from seedant injection) and/or electrical power requirements must be quantified. These effects can have a significant impact on the overall specific impulse performance of the MHD-Fanjet. Furthermore, the group must investigate the best method for electrically uniting the generators and accelerator (ie., the power source and load).

\section{ROCKET MODEL}

A more detailed numerical model of the rectangular geometry LSC-wave heated $\mathrm{H}_{2}$ rocket gas generator is needed. This code should include non-ideal gas behavior and losses due to radiative, convective, and conductive heat transfer. All such effects contribute to reductions in attainable specific impulse.

\section{E. TRAJECTORY ANALYSIS}

The modified sort code is in excellent shape, but can produce results only as good as the engine performance models it includes. Clearly, as the engine-mode models improve, so will the accuracy of trajectory simulations.

At present, the performance of the Apollo Lightcraft combined-cycle engine along an orbital trajectory can be described as none other than outstanding! Average vehicle acceleration is approximately 4-5 G's, which puts the Lightcraft at orbital velocity in approximately four minutes. Beam power can be reduced low as 2.5 billion watts without degrading vehicle performance. Transition 
between engine modes typically occurs at Mach 3,11, and 25. The $\mathrm{LH}_{2}$ propellant requirement for a $185 \mathrm{Km}$ orbit is only $300 \mathrm{Kg}$, or roughly $520 \mathrm{GW}$-sec for this LEO boost mission.

If the $\mathrm{LH}_{2}$ propellant is purchased at a price identical to that of the STS (i.e., $\$ 3.25 / \mathrm{kg}$ ), the bill comes to $\$ 975$; the total beamed-energy charge would total $\$ 2455$, if one assumed the current $\$ 1.7$ cents/Kw-hr that New York State pays for wholesale Canadian hydroelectric power. Therefore the entire round-trip-to-orbit flight aboard an Apollo Lightcraft would cost only $\$ 3430$, or $\$ 686$ per person. It is interesting to note that a round-trip charter flight to the other side of the planet by commercial jet aircraft (e.g. N.Y. to Tokyo) is presently \$1118. Finally, it bears mentioning that in the Post 2020 era, space travel will become commonplace, and the present expensive "standing army "ground crew will be replaced by efficient macro-computers. These "tireless" workers will schedule launch windows, effortlessly deliver beam power from the "space power grid" to hundreds of spacecraft simulataneously, and electronically mail end-of-the-month billing statements to millions of satisfied travelers.

In this future infrastructure, the dominant costs will be for propellant and power. Payload delivery costs to LEO, will finally plunge a factor of 1000 below that of the Space Shuttle Orbiter. Space, the Final Frontier, will no longer be a curiosity to the masees, will now view the Larth from an entirely new perspective - heretofore reserved for only a few brave astronauts in times gone by. 


\section{RUPTRENCES}

1. Bendor, E., "One Dimensional Flow in MHD Generators", Republic Aviation Corporation, Farmingdale N.Y., AIAA Journal, Vol 3, No. 1 , 1965.

2. Bering, M., "Version IV User's Guide for the Simulation and Optimization of Rocket Trajectories Prgm.", McDonnell Douglas Astro. Co., Texas, Transmittal Memo No 1.2-TMFM86028-53, Dec. 1985.

3. Courant, R.,Super Sonic Flow and Shock Waves Springer- Verlag, NY, 1976.

4. Drake, J. H., "Optimum Isothermal Acceleration of a Plasma with Constant Magnetic Field ", Lockheed Missle and Space Co. Palo Alto, Ca, AlAA Journal, Vol 1 No. 9, 1963.

5. Gabriele, G. A. and Beltracchi. T. J., "OPT3.1: A Nonlinear Programming Code Based on the Generalized Reduced Gradient Method ", Design Automation Laboratory, R.P.I., Troy, N.Y., 1987.

6. Hill, P. and Peterson, C., Mechanics and Thermodynamics of Propulsion, Addison-Wesely Publishing, 1965.

7. Holmes, B., "The Mechanical Loads from LSD Waves and Their Simulation- Vol 1, Stanford Research Inst., Report AFWL-TR-75-285, 1976.

8. Jeng, S-M. and Keefer, D. R., "Numerical Study of Laser-Sustained Hydrogen Plasmas in a Forced Convective Flow ", ALAA Paper 86-1524, 1986.

9. Marston,Charles H., "MHD Accelerator Performance for Specified Interaction Parameter", General Electric Co., Philadelphia, Pa., AIAA Journal, Vol 4, No. $11,1966$.

10. Nyberg, G. A., Hill, S. C, Mitty, T. J., Lidke, K., and Poulin, S., "Performance Analysis of a Laser-Powered SSTO Shuttlecraft ", RPI, Troy, N.Y., Feb., 1987, (To be published).

11. Patch, R. W., "Thermodynamic Properties and Theoretical Rocket Performance of Hydrogen to $100,000 \mathrm{~K}$ and $1.01325 \times 10^{8} \mathrm{~N} / \mathrm{m}^{2}{ }^{n}$, Nasa Rpt. SP-3069.

12. Pirri, A. "Theory for Momentum Transfer to a Surface with a High Powered Laser", Phys. Fluids, Vol. 16 no. 9, Sept. 1973.

13. Raizer, Y., "Heating of a Gas by a Powerful Light Pulse", Soviet Phys. JETP, Vol. 21 no. 5, Nov. 1965.

14. Rosa, R. J., Masnetohydrodynamic Energy Conversion, McGraw-Hill, N.Y., 1968.

15 Unpublished data from Marquardt Corporation, Van Nuys, Cal. 1958.

16. Walburn, A.B. and Harner, P., "Oblique Shock Functions at Hypersonic Speeds ", Fairchild Stratos Corp., Aircraft Missles Div., Hagerstown, Md., Space/Aeronautics, February 1963. 
17. White, F., Viscous Fluid Flow, McGraw-Hill, N.Y., 1974, pp. 637-645.

18. Yos J., "Revised Transport Properties for High Temperature Air and its Components ", Avco Systems Division, Lowell, Mass., Technical Report, Nov. 28, 1967.

19. Yos, J., "Transport Properties of Nitrogen, Hydrogen, Oxygen, and Air to 30,000 K", Research \& Development Div., AVCO Corporation, Wilmington, Mass., Technical Memorandum RAD-TM-63-7.

20. Zel'dovich B., Theory of Detonation, Academic Press, NY, 1960. 
APPENDIX:

THRED-DIMENSIONAL MODELING OF APOLLO LIGHTCRAFT LOFTLINES 


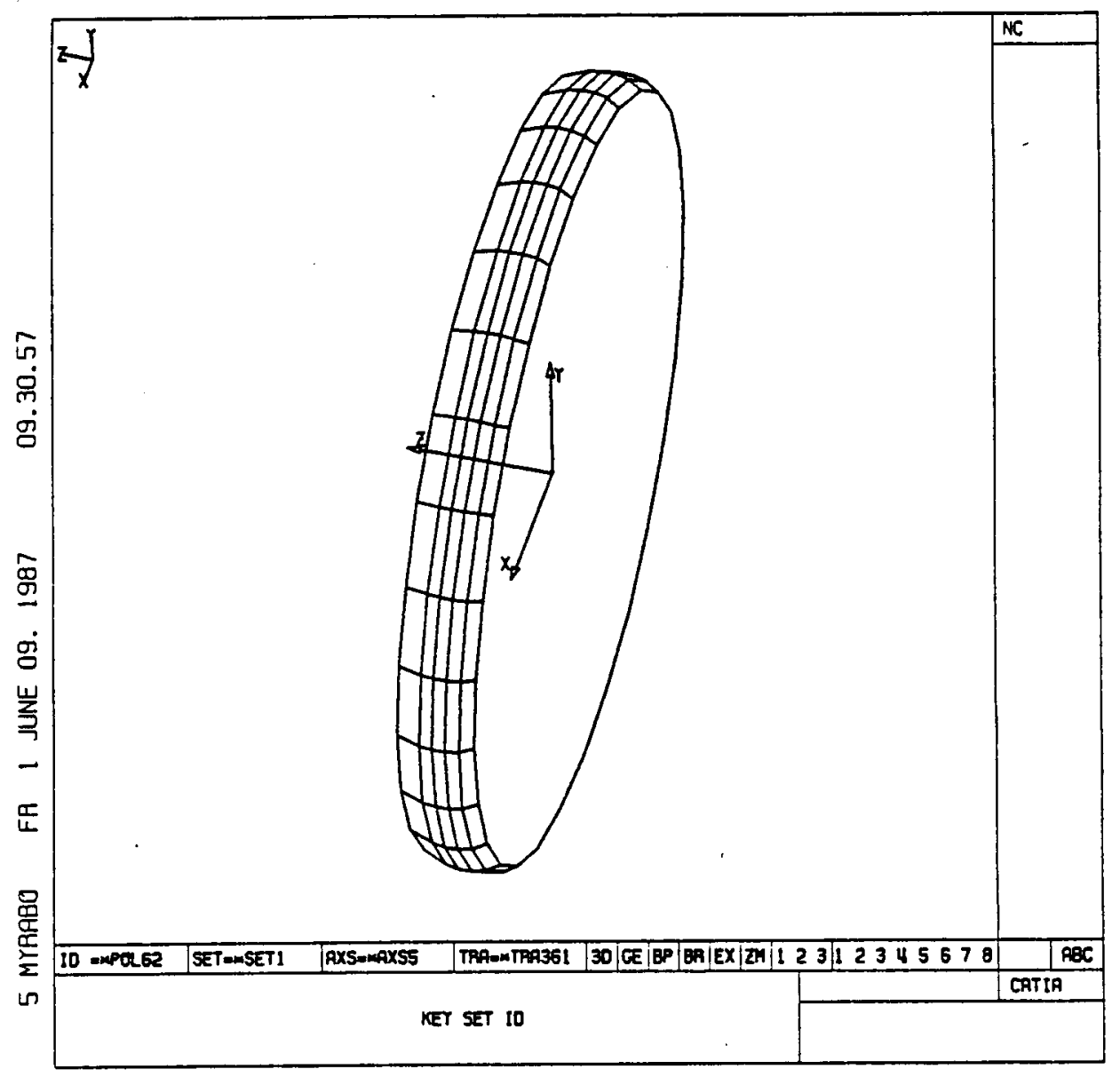

1

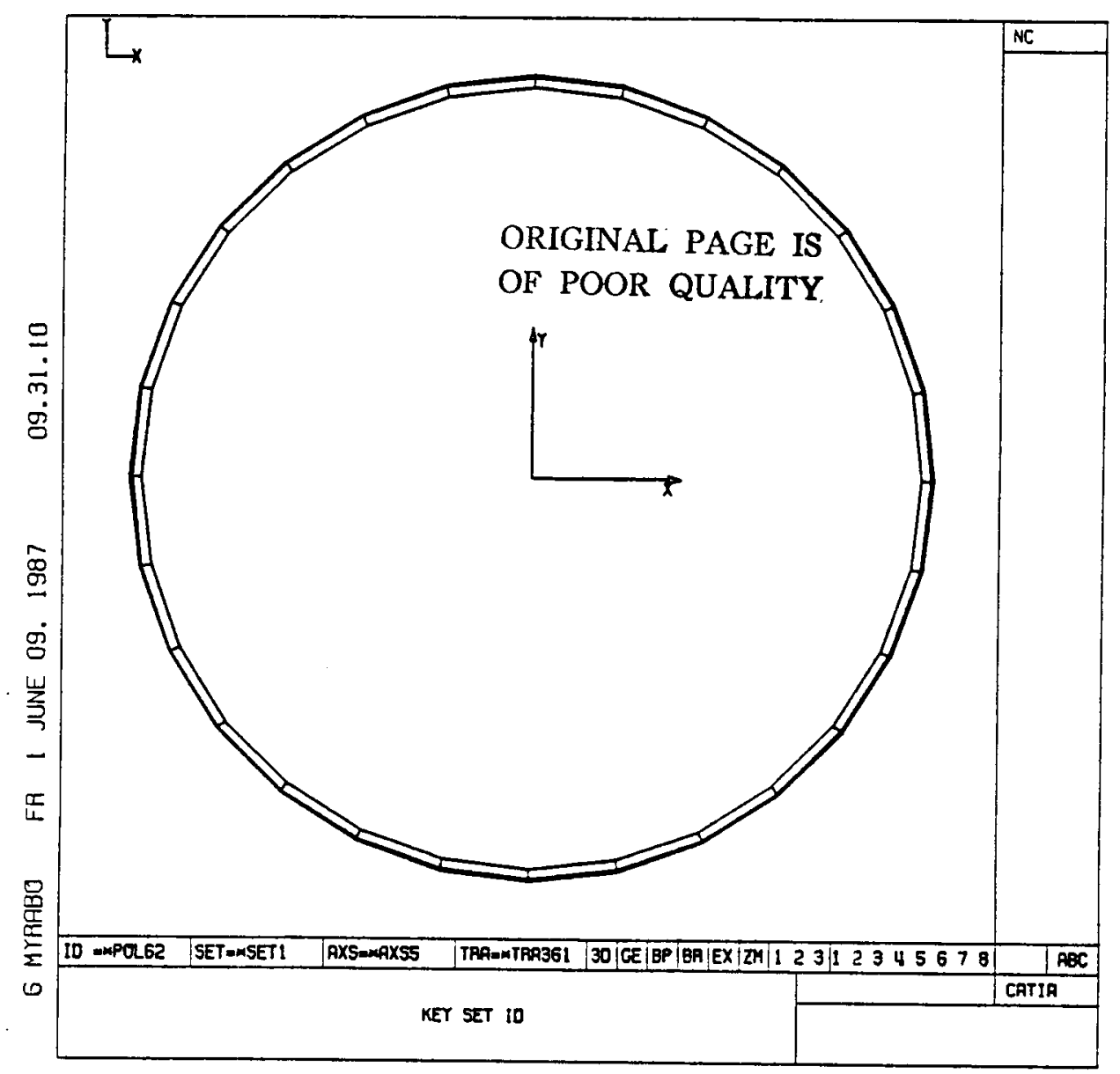



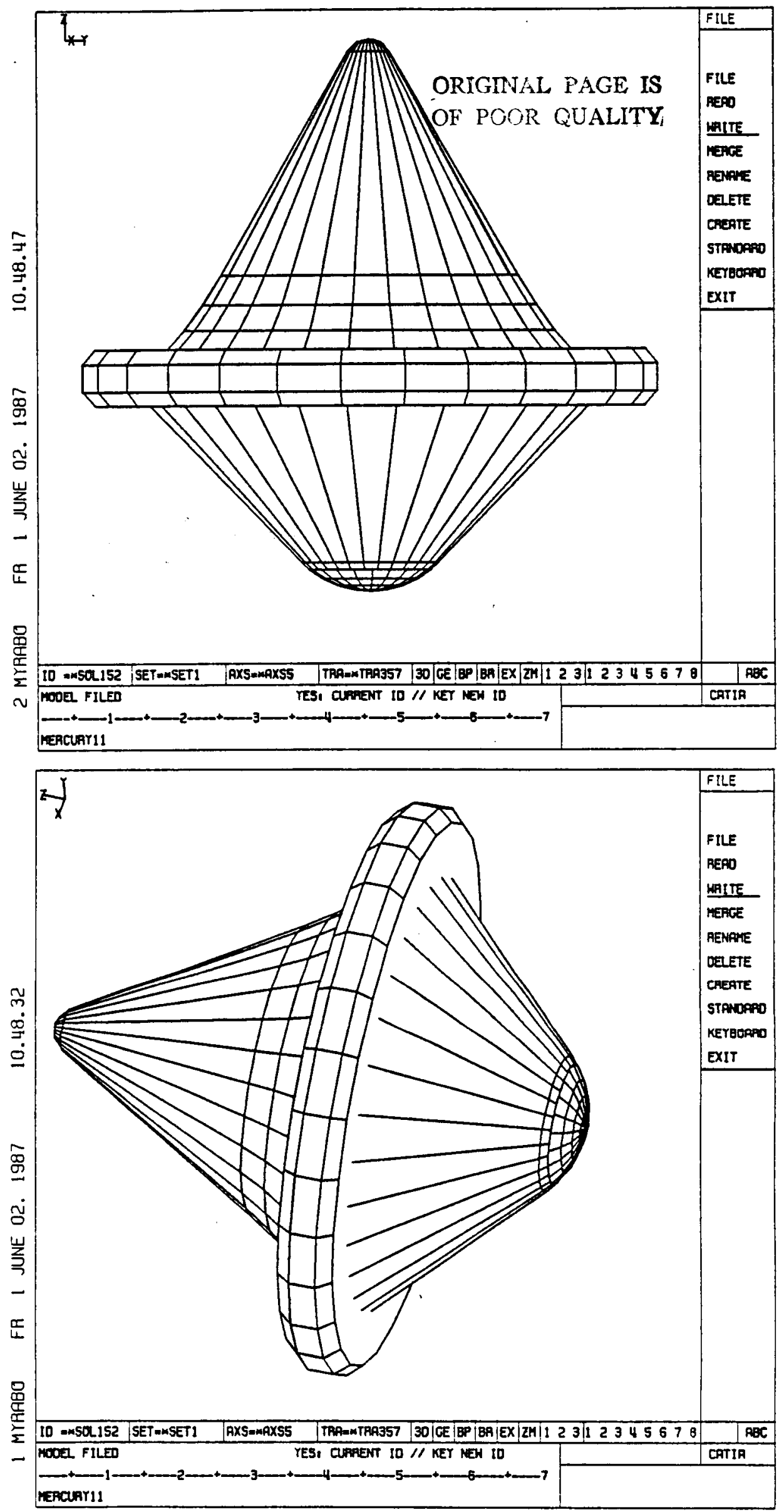

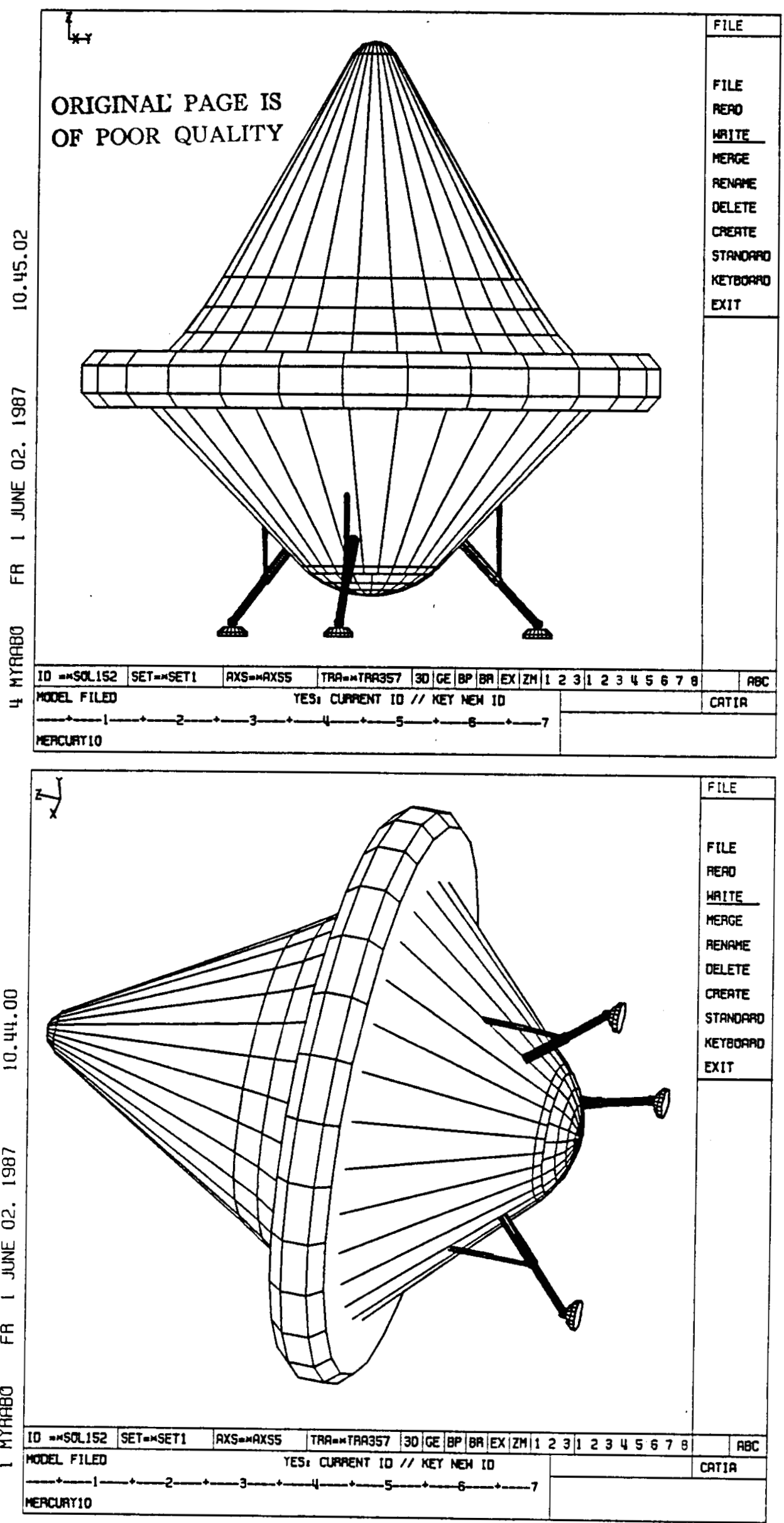

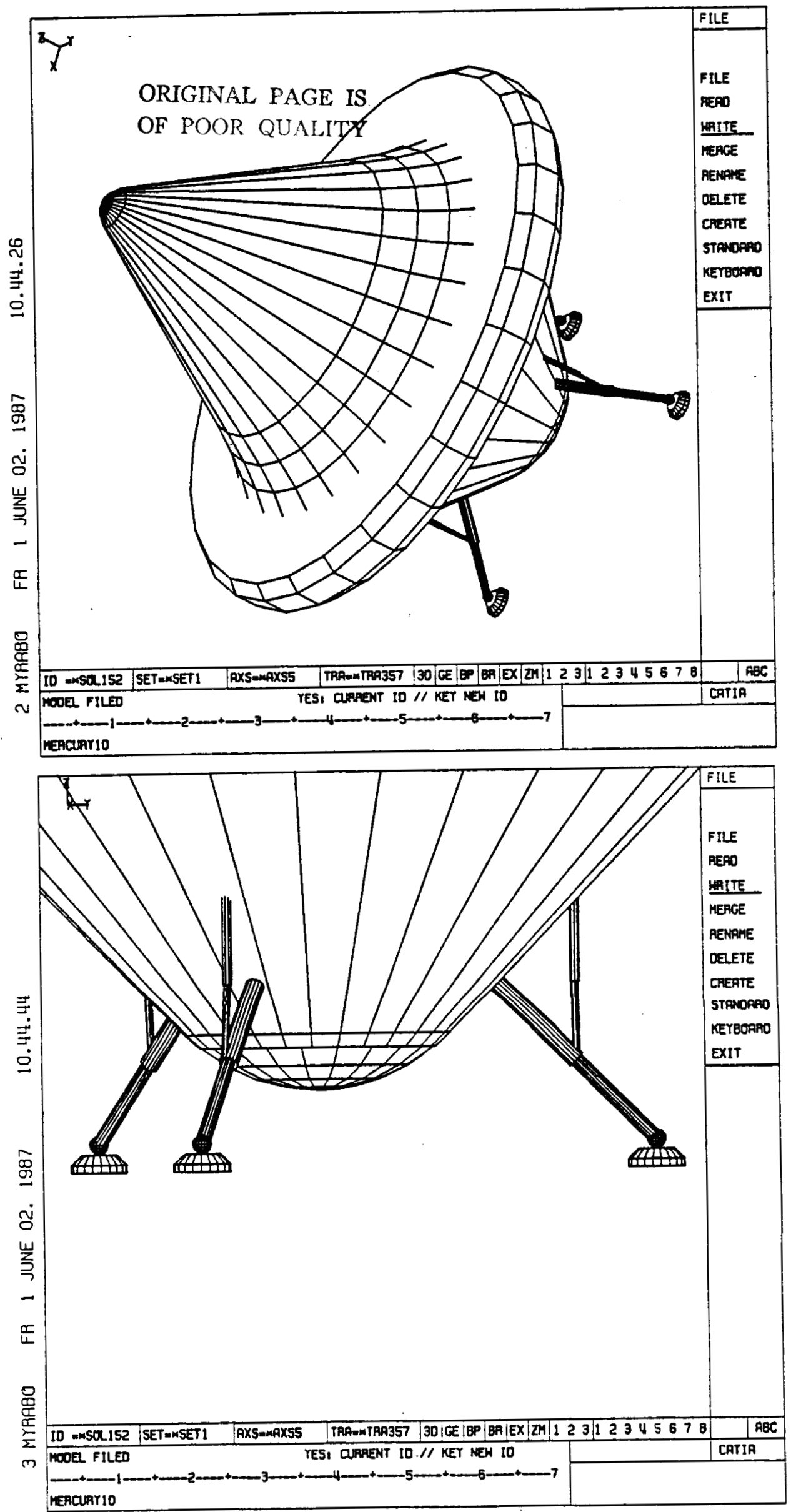

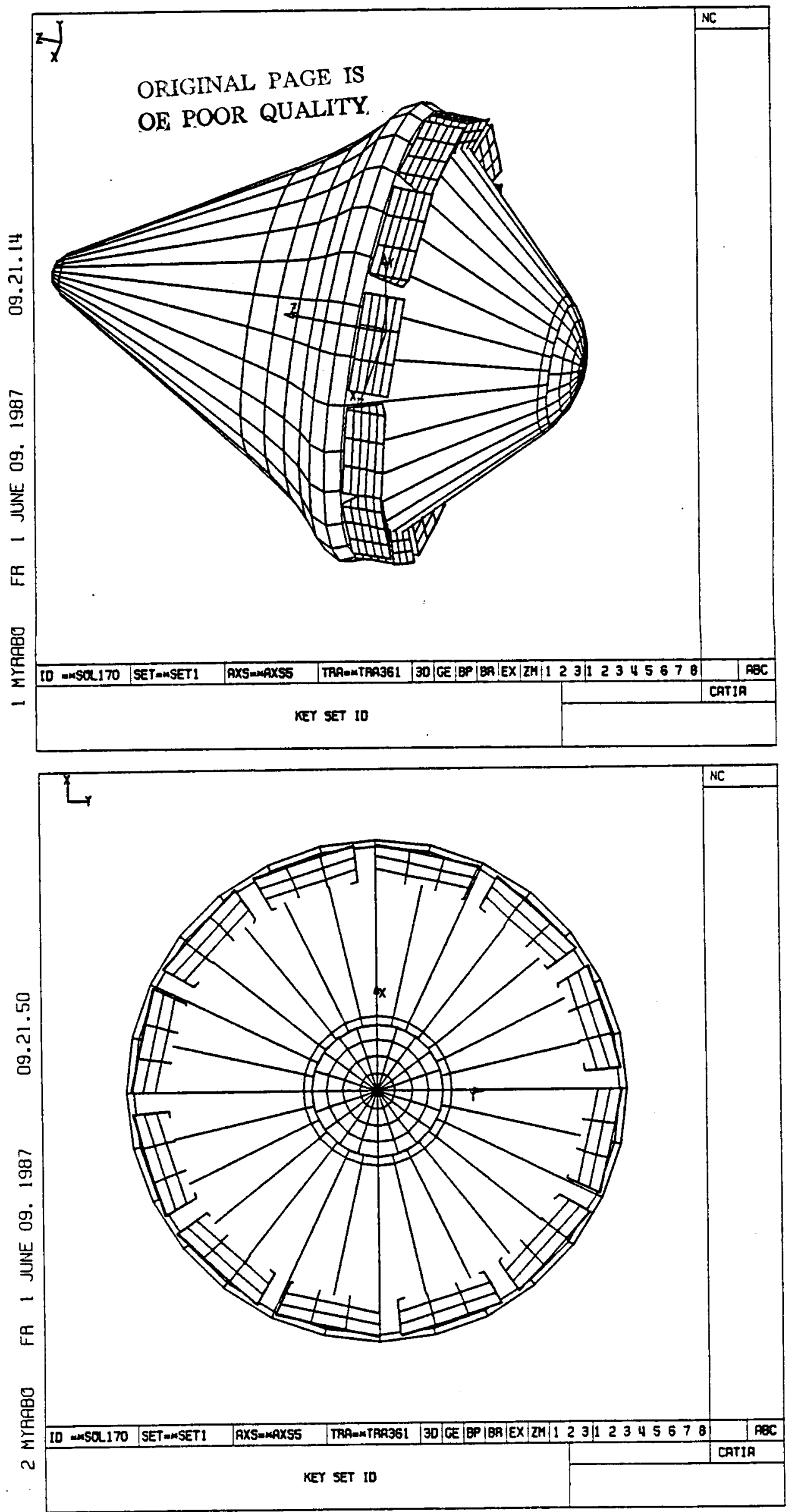

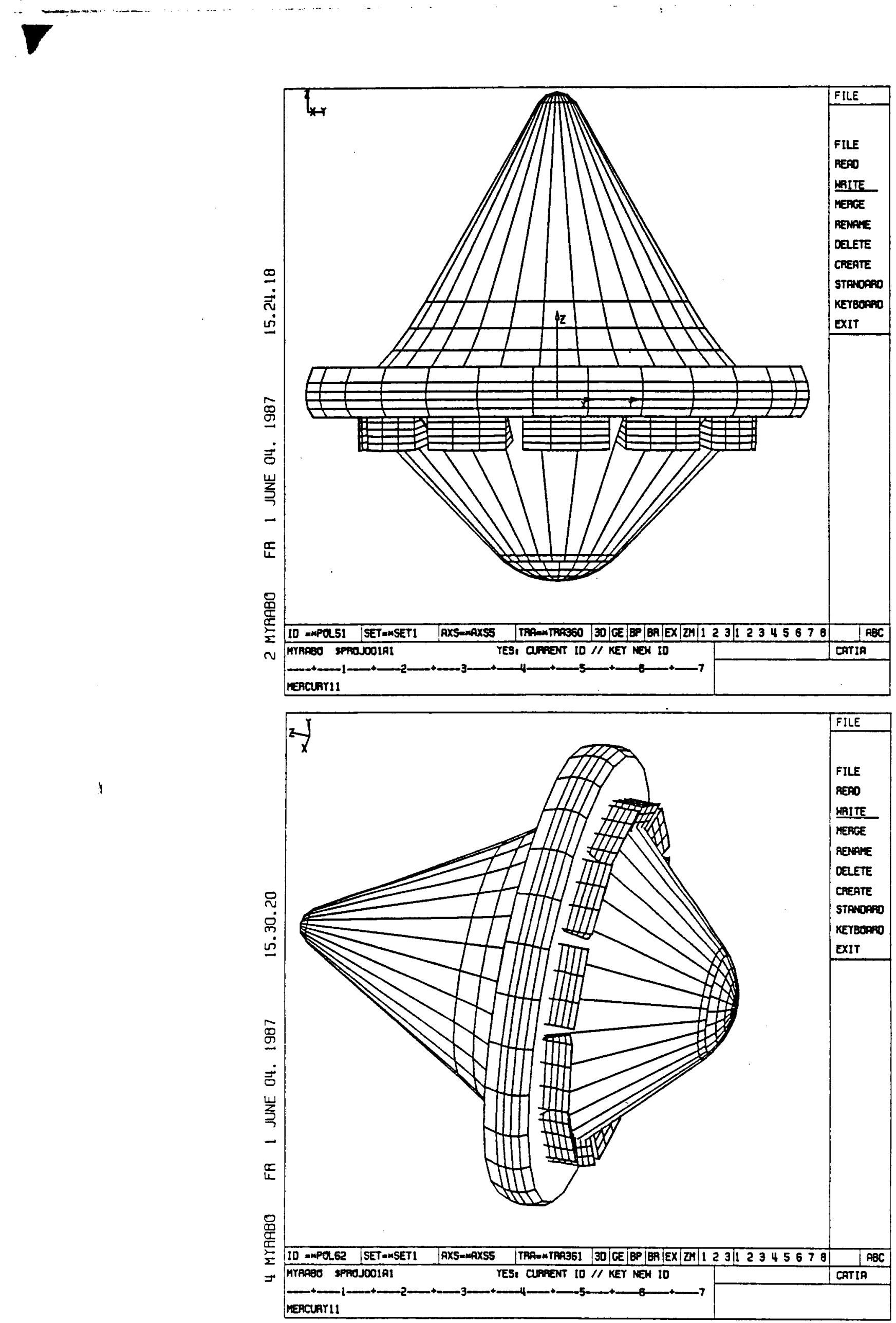

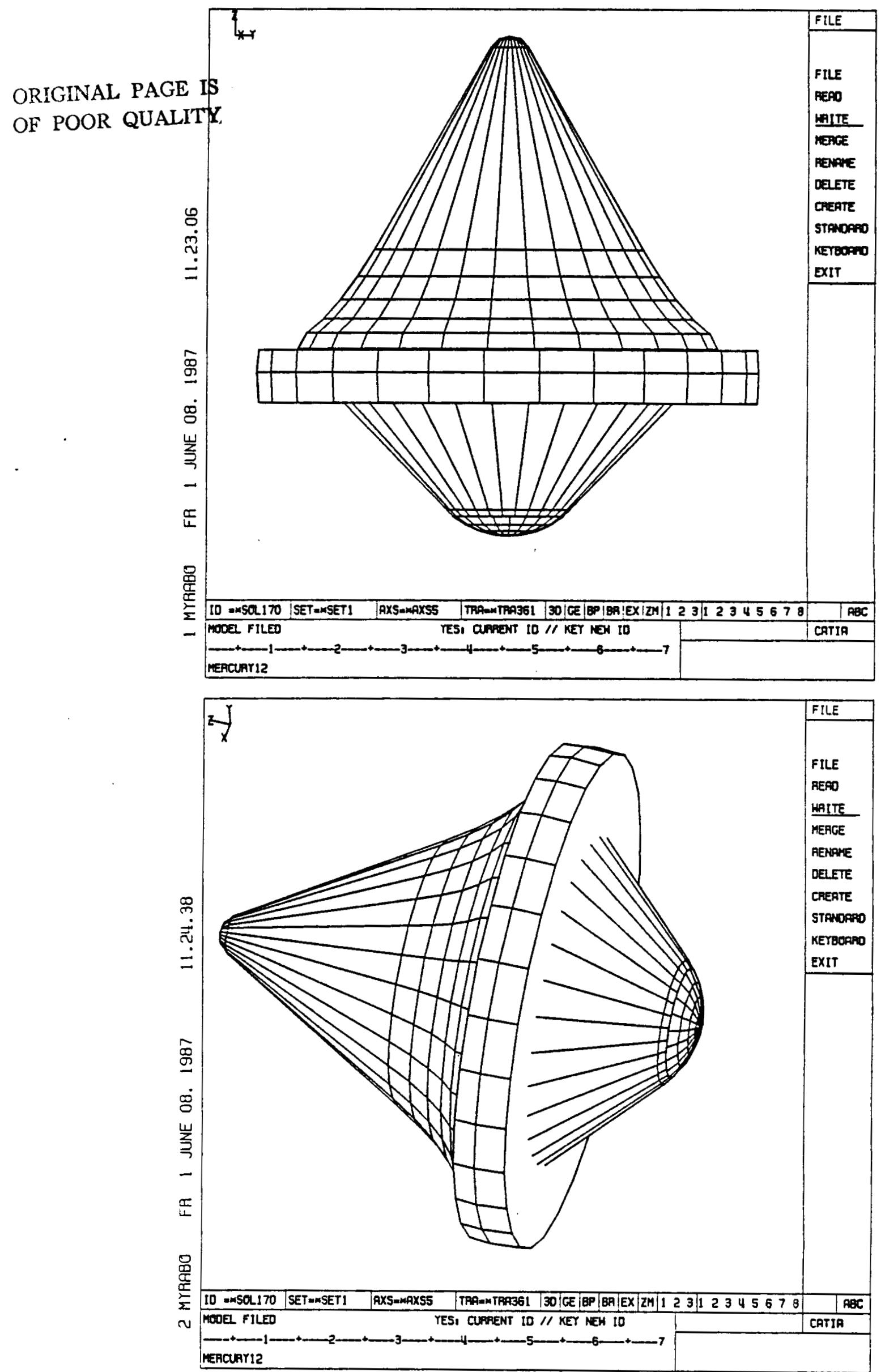


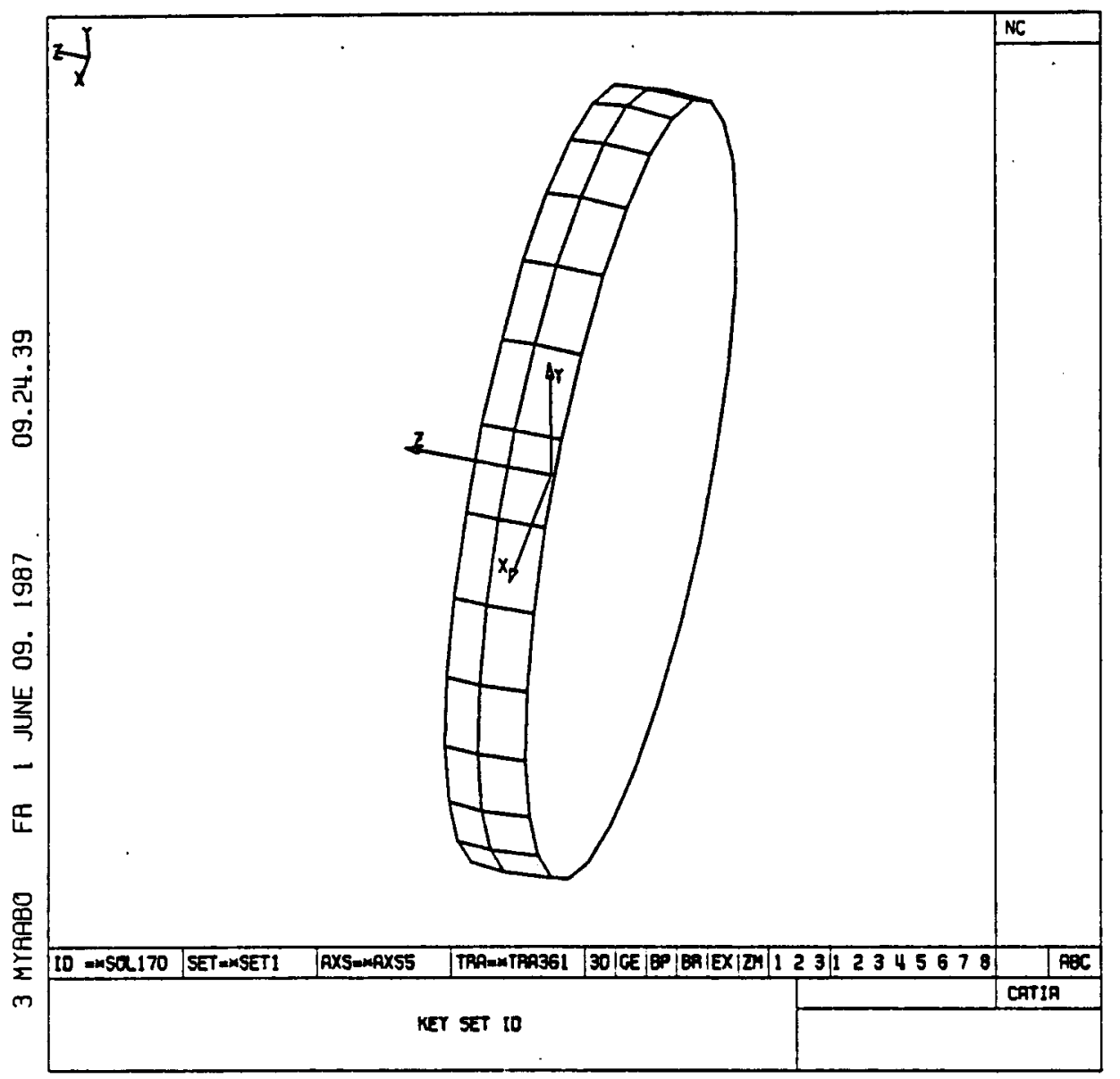

END

DATE

PAAY 18,1988

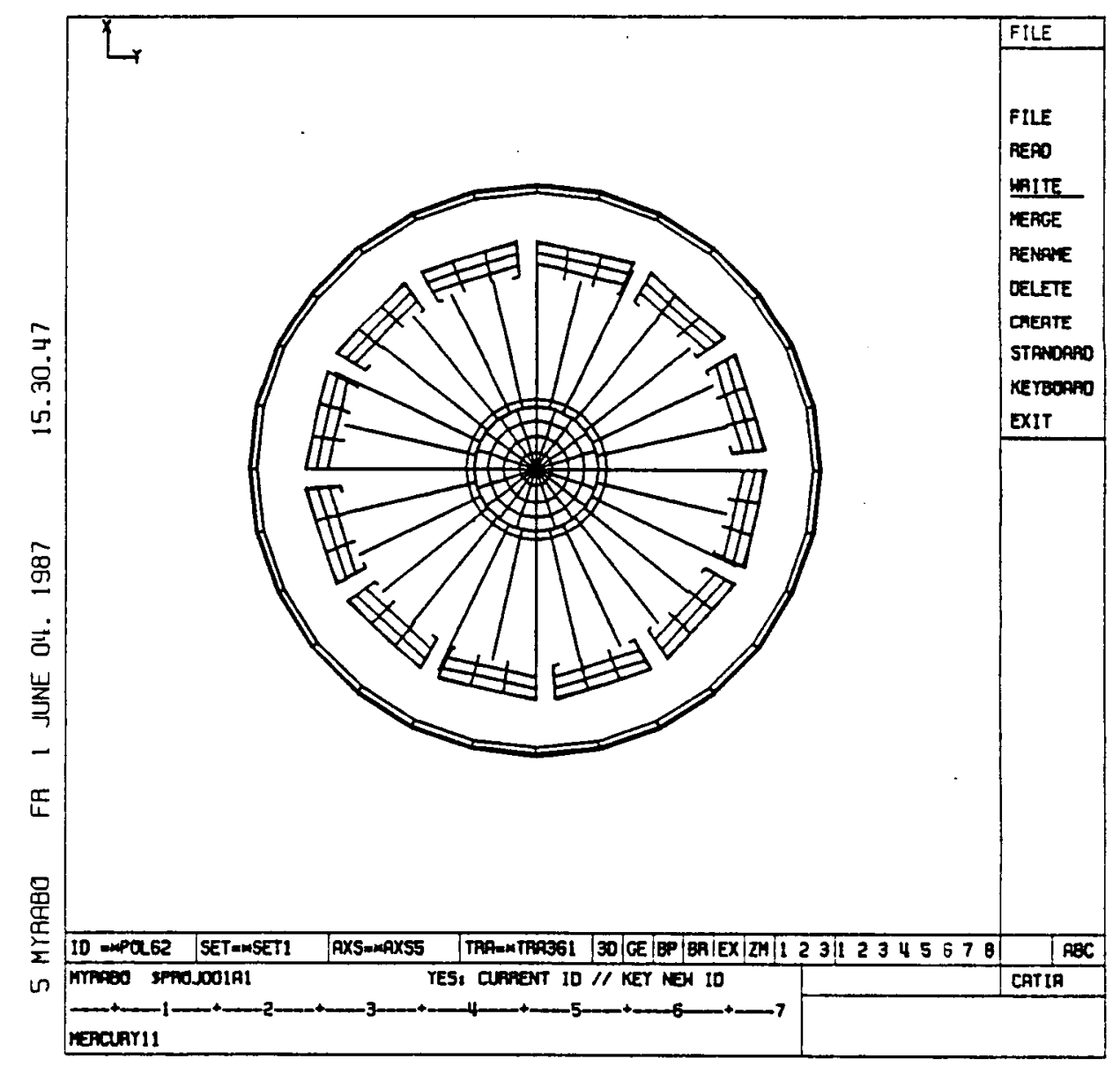

